\title{
The algebraic classification of nilpotent algebras 1
}

\author{
Ivan Kaygorodov ${ }^{a}$, Mykola Khrypchenko $^{b}$ \& Samuel A. Lopes ${ }^{c}$ \\ ${ }^{a}$ CMCC, Universidade Federal do ABC, Santo André, Brazil \\ ${ }^{b}$ Departamento de Matemática, Universidade Federal de Santa Catarina, Florianópolis, Brazil \\ ${ }^{c}$ CMUP, Faculdade de Ciências, Universidade do Porto, Rua do Campo Alegre 687, 4169-007 Porto, Portugal \\ E-mail addresses: \\ Ivan Kaygorodov (kaygorodov.ivan@gmail.com) \\ Mykola Khrypchenko (nskhripchenko@gmail.com) \\ Samuel A. Lopes (slopes@fc.up.pt)
}

\begin{abstract}
We give the complete algebraic classification of all complex 4-dimensional nilpotent algebras. The final list has 234 (parametric families of) isomorphism classes of algebras, 66 of which are new in the literature.
\end{abstract}

Keywords: Nilpotent algebra, algebraic classification, central extension.

MSC2010: 17A30, 17A01, 17D99.

\section{INTRODUCTION}

The description, up to isomorphism, of all the algebras of some fixed dimension satisfying certain properties (the so-called algebraic classification) is a classical problem in algebra. There are many papers devoted to algebraic classification of small-dimensional algebras in several varieties of associative and non-associative algebras [1-8, 11-16, 18, 19]. Another interesting direction in the classification of algebras is the geometric classification (see, [11, 16, 18] and references therein). Restricting our consideration to subvarieties of complex nilpotent 4-dimensional algebras, we mention here the results on associative [7], commutative [11], bicommutative [18], Leibniz [8], terminal [16] and $\mathfrak{C} \mathfrak{D}$-algebras [14]. In the present paper, we complete the algebraic classification of allcomplex 4dimensional nilpotent algebras. Namely, we find 66 new algebras and parametric families of algebras completing the list of 4-dimensional nilpotent algebras initiated in the above mentioned works.

Our approach is based on the calculation of central extensions of nilpotent algebras of dimension less than 4. This method was developed by Skjelbred and Sund for Lie algebras in [20] and has been an important tool for years (see, for example, [13, 17, 20] and references therein).

\footnotetext{
${ }^{1}$ The work was partially supported by CNPq 404649/2018-1, 302980/2019-9; RFBR 20-01-00030; by the Fundação para a Ciência e a Tecnologia (Portuguese Foundation for Science and Technology) through the project PTDC/MATPUR/31174/2017; by CMUP, which is financed by national funds through FCT_Fundação para a Ciência e a Tecnologia, I.P., under the project with reference UIDB/00144/2020.
} 
In Section 1.1 we give a brief description of the method of central extensions and its adaptation to our case. It turns out that most of the nilpotent 4 -dimensional algebras are $\mathfrak{C} \mathfrak{D}$-algebras, which were classified in [14]. Our classification will thus be carried out modulo this subvariety of 4-dimensional nilpotent algebras, as explained in Section 1.1.1. In Section 1.2 we discuss several other useful classes of nilpotent algebras of dimension at most 4. In particular, we give a list of the 3-dimensional nilpotent algebras (they are all, in fact, $\mathfrak{C} \mathfrak{D}$-algebras). The remainder of Section 1 (Sections 1.3 1.6) is devoted to the construction and classification of 1-dimensional non- $\mathfrak{C} \mathfrak{D}$ central extensions of 3-dimensional nilpotent algebras. The work done in Section 1 culminates in the full classification of 4-dimensional nilpotent algebras, which is completed in Section 2, where we present the full list of representatives of isomorphism classes. Finally, in Section 3 we present a few applications of our results to the classes of Lie-admissible and Alia type algebras.

Main result. The main result of the paper is Theorem 2 giving a full classification of complex 4dimensional nilpotent algebras. The complete list of non-isomorphic algebras consists of four parts:

(1) trivial $\mathfrak{C} \mathfrak{D}$-algebras, which were classified in [8, Theorem 2.1, Theorem 2.3 and Theorem 2.5], the only anticommutative trivial $\mathfrak{C} \mathfrak{D}$-algebra being $\mathfrak{C} \mathfrak{D}_{03}^{4 *}$;

(2) terminal non-trivial extensions of the family $\mathfrak{C} \mathfrak{D}_{04}^{3 *}(\lambda)$, which were classified in [16, 1.4.5. 1-dimensional central extensions of $\mathbf{T}_{04}^{3 *}$;

(3) non-trivial non-terminal $\mathfrak{C} \mathfrak{D}$-algebras, which were classified in [14];

(4) the nilpotent algebras found in Sections $1.3-1.6$ of the present paper.

\section{The ALGEBRAiC CLASSIFICATION OF NILPOTENT ALGEBRAS}

1.1. Method of classification of nilpotent algebras. Let $\mathbf{A}$ be an algebra, $\mathbf{V}$ a vector space and $\mathrm{Z}^{2}(\mathbf{A}, \mathbf{V}) \cong \operatorname{Hom}(\mathbf{A} \otimes \mathbf{A}, \mathbf{V})$ denote the space of bilinear maps $\theta: \mathbf{A} \times \mathbf{A} \longrightarrow \mathbf{V}$. For $f \in$ $\operatorname{Hom}(\mathbf{A}, \mathbf{V})$, we introduce $\delta f \in \mathrm{Z}^{2}(\mathbf{A}, \mathbf{V})$ by the equality $\delta f(x, y)=f(x y)$ and define $\mathrm{B}^{2}(\mathbf{A}, \mathbf{V})=$ $\{\delta f \mid f \in \operatorname{Hom}(\mathbf{A}, \mathbf{V})\}$. One can easily check that $\mathrm{B}^{2}(\mathbf{A}, \mathbf{V})$ is a linear subspace of $\mathrm{Z}^{2}(\mathbf{A}, \mathbf{V})$. Let us define $\mathrm{H}^{2}(\mathbf{A}, \mathbf{V})$ as the quotient space $\mathrm{Z}^{2}(\mathbf{A}, \mathbf{V}) / \mathrm{B}^{2}(\mathbf{A}, \mathbf{V})$. The equivalence class of $\theta \in$ $\mathrm{Z}^{2}(\mathbf{A}, \mathbf{V})$ in $\mathrm{H}^{2}(\mathbf{A}, \mathbf{V})$ is denoted by $[\theta]$.

Suppose now that $\operatorname{dim} \mathbf{A}=m<n$ and $\operatorname{dim} \mathbf{V}=n-m$. For any bilinear map $\theta: \mathbf{A} \times$ $\mathbf{A} \longrightarrow \mathbf{V}$, one can define on the space $\mathbf{A}_{\theta}:=\mathbf{A} \oplus \mathbf{V}$ the bilinear product $[-,-]_{\mathbf{A}_{\theta}}$ by the equality $\left[x+x^{\prime}, y+y^{\prime}\right]_{\mathbf{A}_{\theta}}=x y+\theta(x, y)$ for $x, y \in \mathbf{A}, x^{\prime}, y^{\prime} \in \mathbf{V}$. The algebra $\mathbf{A}_{\theta}$ is called an $(n-m)$ dimensional central extension of $\mathbf{A}$ by $\mathbf{V}$. It is also clear that $\mathbf{A}_{\theta}$ is nilpotent if and only if $\mathbf{A}$ is.

For a bilinear map $\theta: \mathbf{A} \times \mathbf{A} \longrightarrow \mathbf{V}$, the space $\theta^{\perp}=\{x \in \mathbf{A} \mid \theta(x, \mathbf{A})=\theta(\mathbf{A}, x)=0\}$ is called the annihilator of $\theta$. For an algebra $\mathbf{A}$, the ideal Ann $(\mathbf{A})=\{x \in \mathbf{A} \mid x \mathbf{A}=\mathbf{A} x=0\}$ is called the annihilator of $\mathbf{A}$. One has

$$
\operatorname{Ann}\left(\mathbf{A}_{\theta}\right)=\left(\theta^{\perp} \cap \operatorname{Ann}(\mathbf{A})\right) \oplus \mathbf{V} .
$$

Any $n$-dimensional algebra with non-trivial annihilator can be represented in the form $\mathbf{A}_{\theta}$ for some $m$-dimensional algebra $\mathbf{A}$, an $(n-m)$-dimensional vector space $\mathbf{V}$ and $\theta \in \mathrm{Z}^{2}(\mathbf{A}, \mathbf{V})$, where $m<n$ (see [13, Lemma 5]). Moreover, there is a unique such representation with $m=n-\operatorname{dim} \operatorname{Ann}(\mathbf{A})$. Note also that the latter equality is equivalent to the condition $\theta^{\perp} \cap \operatorname{Ann}(\mathbf{A})=0$. 
Let us pick some $\phi \in \operatorname{Aut}(\mathbf{A})$, where $\operatorname{Aut}(\mathbf{A})$ is the automorphism group of $\mathbf{A}$. For $\theta \in$ $\mathrm{Z}^{2}(\mathbf{A}, \mathbf{V})$, let us define $(\phi \theta)(x, y)=\theta(\phi(x), \phi(y))$. Then we get an action of Aut $(\mathbf{A})$ on $\mathrm{Z}^{2}(\mathbf{A}, \mathbf{V})$ that induces an action of the same group on $\mathrm{H}^{2}(\mathbf{A}, \mathbf{V})$.

Definition 1. Let $\mathbf{A}$ be an algebra and $I$ be a subspace of $\operatorname{Ann}(\mathbf{A})$. If $\mathbf{A}=\mathbf{A}_{0} \oplus I$, for some subalgebra $\mathbf{A}_{0}$ of $\mathbf{A}$, then $I$ is called an annihilator component of $\mathbf{A}$. We say that $\mathbf{A}$ is split if it has some non-zero annihilator component; otherwise we say that $\mathbf{A}$ is non-split.

For a linear space $\mathbf{U}$, the Grassmannian $G_{s}(\mathbf{U})$ is the set of all $s$-dimensional linear subspaces of $\mathbf{U}$. For any $s \geq 1$, the action of $\operatorname{Aut}(\mathbf{A})$ on $\mathrm{H}^{2}(\mathbf{A}, \mathbb{C})$ induces an action of the same group on $G_{s}\left(H^{2}(\mathbf{A}, \mathbb{C})\right)$. Let us define

$$
\mathbf{T}_{s}(\mathbf{A})=\left\{\mathbf{W} \in G_{s}\left(\mathrm{H}^{2}(\mathbf{A}, \mathbb{C})\right) \mid \underset{[\theta] \in W}{\cap} \theta^{\perp} \cap \operatorname{Ann}(\mathbf{A})=0\right\} .
$$

Note that $\mathbf{T}_{s}(\mathbf{A})$ is stable under the action of $\operatorname{Aut}(\mathbf{A})$.

Let us fix a basis $e_{1}, \ldots, e_{s}$ of $\mathbf{V}$ and $\theta \in Z^{2}(\mathbf{A}, \mathbf{V})$. Then there are unique $\theta_{i} \in Z^{2}(\mathbf{A}, \mathbb{C})(1 \leq i \leq$ $s$ ) such that $\theta(x, y)=\sum_{i=1}^{s} \theta_{i}(x, y) e_{i}$ for all $x, y \in \mathbf{A}$. Note that $\theta^{\perp}=\theta_{1}^{\perp} \cap \theta_{2}^{\perp} \cdots \cap \theta_{s}^{\perp}$ in this case. If $\theta^{\perp} \cap \operatorname{Ann}(\mathbf{A})=0$, then by [13, Lemma 13] the algebra $\mathbf{A}_{\theta}$ is split if and only if $\left[\theta_{1}\right],\left[\theta_{2}\right], \ldots,\left[\theta_{s}\right]$ are linearly dependent in $\mathrm{H}^{2}(\mathbf{A}, \mathbb{C})$. Thus, if $\theta^{\perp} \cap \operatorname{Ann}(\mathbf{A})=0$ and $\mathbf{A}_{\theta}$ is non-split, then $\left\langle\left[\theta_{1}\right], \ldots,\left[\theta_{s}\right]\right\rangle$ is an element of $\mathbf{T}_{s}(\mathbf{A})$. Now, if $\vartheta \in Z^{2}(\mathbf{A}, \mathbf{V})$ is such that $\vartheta^{\perp} \cap \operatorname{Ann}(\mathbf{A})=0$ and $\mathbf{A}_{\vartheta}$ is non-split, then by [13, Lemma 17] one has $\mathbf{A}_{\vartheta} \cong \mathbf{A}_{\theta}$ if and only if $\left\langle\left[\theta_{1}\right],\left[\theta_{2}\right], \ldots,\left[\theta_{s}\right]\right\rangle,\left\langle\left[\vartheta_{1}\right],\left[\vartheta_{2}\right], \ldots,\left[\vartheta_{s}\right]\right\rangle \in$ $\mathbf{T}_{s}(\mathbf{A})$ belong to the same orbit under the action of $\operatorname{Aut}(\mathbf{A})$, where $\vartheta(x, y)=\sum_{i=1}^{s} \vartheta_{i}(x, y) e_{i}$.

Hence, there is a one-to-one correspondence between the set of Aut (A)-orbits on $\mathbf{T}_{s}(\mathbf{A})$ and the set of isomorphism classes of central extensions of $\mathbf{A}$ by $\mathbf{V}$ with $s$-dimensional annihilator and trivial annihilator component. Consequently, to construct all $s$-dimensional central extensions with trivial annihilator component of a given $(n-s)$-dimensional algebra $\mathbf{A}$ one has to describe $\mathbf{T}_{s}(\mathbf{A})$, Aut $(\mathbf{A})$ and the action of $\operatorname{Aut}(\mathbf{A})$ on $\mathbf{T}_{s}(\mathbf{A})$ and then for each orbit under the action of $\operatorname{Aut}(\mathbf{A})$ on $\mathbf{T}_{s}(\mathbf{A})$ pick a representative and construct the algebra corresponding to it.

1.1.1. Reduction to non-CD-algebras. The class of $\mathfrak{C} \mathfrak{D}$-algebras is defined by the property that the commutator of any pair of multiplication operators is a derivation [1,14]; namely, an algebra $\mathfrak{A}$ is a $\mathfrak{C} \mathfrak{D}$-algebra if and only if

$$
\left[T_{x}, T_{y}\right] \in \mathfrak{D e r}(\mathfrak{A})
$$

for all $x, y \in \mathfrak{A}$, where $T_{z} \in\left\{R_{z}, L_{z}\right\}$. Here we use the notation $R_{z}$ (resp. $L_{z}$ ) for the operator of right (resp. left) multiplication in $\mathfrak{A}$. We will denote the variety of $\mathfrak{C} \mathfrak{D}$-algebras by $\mathfrak{C} \mathfrak{D}$. In terms of identities, the class of $\mathfrak{C} \mathfrak{D}$-algebras is defined by the following three:

$$
\begin{aligned}
& ((x y) a) b-((x y) b) a=((x a) b-(x b) a) y+x((y a) b-(y b) a), \\
& (a(x y)) b-a((x y) b)=((a x) b-a(x b)) y+x((a y) b-a(y b)), \\
& a(b(x y))-b(a(x y))=(a(b x)-b(a x)) y+x(a(b y)-b(a y)) .
\end{aligned}
$$


Our method of classification of nilpotent algebras will be based on a classification of $\mathfrak{C} \mathfrak{D}$ -

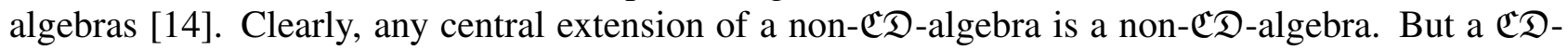
algebra may have extensions which are not $\mathfrak{C} \mathfrak{D}$-algebras. More precisely, let $\mathbf{A}$ be a $\mathfrak{C} \mathfrak{D}$-algebra and $\theta \in Z^{2}(\mathbf{A}, \mathbb{C})$. Then $\mathbf{A}_{\theta}$ is a $\mathfrak{C} \mathfrak{D}$-algebra if and only if

$$
\begin{aligned}
& \theta((x y) a, b)-\theta((x y) b, a)=\theta((x a) b-(x b) a, y)+\theta(x,(y a) b-(y b) a), \\
& \theta(a(x y), b)-\theta(a,(x y) b)=\theta((a x) b-a(x b), y)+\theta(x,(a y) b-a(y b)), \\
& \theta(a, b(x y))-\theta(b, a(x y))=\theta(a(b x)-b(a x), y)+\theta(x, a(b y)-b(a y)) .
\end{aligned}
$$

for all $x, y, z \in \mathbf{A}$. Define the subspace $\mathrm{Z}_{\mathfrak{C D}}^{2}(\mathbf{A}, \mathbb{C})$ of $\mathrm{Z}^{2}(\mathbf{A}, \mathbb{C})$ by

$$
\mathrm{Z}_{\mathfrak{C D}}^{2}(\mathbf{A}, \mathbb{C})=\left\{\theta \in \mathrm{Z}^{2}(\mathbf{A}, \mathbb{C}): \theta \text { satisfies (11) (2) and (3) }\right\} \text {. }
$$

Observe that $\mathrm{B}^{2}(\mathbf{A}, \mathbb{C}) \subseteq \mathrm{Z}_{\mathfrak{C D}}^{2}(\mathbf{A}, \mathbb{C})$. Let $\mathrm{H}_{\mathfrak{C} \mathfrak{D}}^{2}(\mathbf{A}, \mathbb{C})=\mathrm{Z}_{\mathfrak{C} \mathfrak{D}}^{2}(\mathbf{A}, \mathbb{C}) / \mathrm{B}^{2}(\mathbf{A}, \mathbb{C})$. Then $\mathrm{H}_{\mathfrak{C D}}^{2}(\mathbf{A}, \mathbb{C})$ is a subspace of $\mathrm{H}^{2}(\mathbf{A}, \mathbb{C})$. Define

$$
\begin{aligned}
& \mathbf{R}_{s}(\mathbf{A})=\left\{\mathbf{W} \in \mathbf{T}_{\mathbf{s}}(\mathbf{A}): \mathbf{W} \in \mathbf{G}_{\mathbf{s}}\left(\mathrm{H}_{\mathfrak{C D}}^{2}(\mathbf{A}, \mathbb{C})\right)\right\}, \\
& \mathbf{U}_{s}(\mathbf{A})=\left\{\mathbf{W} \in \mathbf{T}_{\mathbf{s}}(\mathbf{A}): \mathbf{W} \notin \mathbf{G}_{\mathbf{s}}\left(\mathrm{H}_{\mathfrak{C D}}^{2}(\mathbf{A}, \mathbb{C})\right)\right\} .
\end{aligned}
$$

Then $\mathbf{T}_{s}(\mathbf{A})=\mathbf{R}_{\mathbf{s}}(\mathbf{A}) \cup \mathbf{U}_{s}(\mathbf{A})$. The sets $\mathbf{R}_{s}(\mathbf{A})$ and $\mathbf{U}_{s}(\mathbf{A})$ are stable under the action of Aut $(\mathbf{A})$. Thus, the nilpotent algebras corresponding to the representatives of $\operatorname{Aut}(\mathbf{A})$-orbits on $\mathbf{R}_{s}(\mathbf{A})$ are $\mathfrak{C D}$-algebras, while those corresponding to the representatives of $\operatorname{Aut}(\mathbf{A})$-orbits on $\mathbf{U}_{s}(\mathbf{A})$ are non-

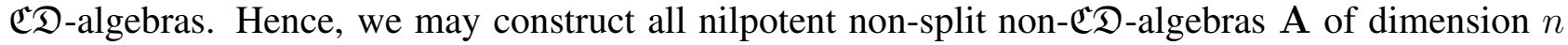
with $s$-dimensional annihilator from a given nilpotent algebra $\mathbf{A}^{\prime}$ of dimension $n-s$ as follows:

(1) If $\mathbf{A}^{\prime}$ is non- $\mathfrak{C} \mathfrak{D}$, then apply the procedure.

(2) Otherwise, do the following:

(a) Determine $\mathbf{U}_{s}\left(\mathbf{A}^{\prime}\right)$ and $\operatorname{Aut}\left(\mathbf{A}^{\prime}\right)$.

(b) Determine the set of $\operatorname{Aut}\left(\mathbf{A}^{\prime}\right)$-orbits on $\mathbf{U}_{s}\left(\mathbf{A}^{\prime}\right)$.

(c) For each orbit, construct the nilpotent algebra corresponding to one of its representatives.

We will use the following auxiliary notation during the construction of central extensions. Let $\mathbf{A}$ be an algebra with basis $e_{1}, e_{2}, \ldots, e_{n}$. Then $\Delta_{i j}: \mathbf{A} \times \mathbf{A} \longrightarrow \mathbb{C}$ denotes the bilinear form defined by the equalities $\Delta_{i j}\left(e_{i}, e_{j}\right)=1$ and $\Delta_{i j}\left(e_{l}, e_{m}\right)=0$ for $(l, m) \neq(i, j)$. In this case, the $\Delta_{i j}$ with $1 \leq i, j \leq n$ form a basis of the space of bilinear forms on $\mathbf{A}$.

We also denote by

$$
\begin{array}{ll}
\mathfrak{C} \mathfrak{D}_{j}^{i *} & \text { the } j \text { th } i \text {-dimensional nilpotent trivial } \mathfrak{C} \mathfrak{D} \text {-algebra, } \\
\mathfrak{C} \mathfrak{D}_{j}^{i} & \text { the } j \text { th } i \text {-dimensional nilpotent non-trivial } \mathfrak{C} \mathfrak{D} \text {-algebra, } \\
\mathbf{D}_{j}^{i} & \text { the } j \text { th } i \text {-dimensional nilpotent terminal algebra, } \\
\mathbf{N}_{j}^{i} & \text { the } j \text { th } i \text {-dimensional nilpotent non- } \mathfrak{C} \mathfrak{D} \text {-algebra. }
\end{array}
$$

\subsection{Some special types of nilpotent algebras.}

1.2.1. Trivial $\mathfrak{C} \mathfrak{D}$-algebras. Recall that the class of $n$-dimensional algebras defined by the identities $(x y) z=0$ and $x(y z)=0$ lies in the intersection of all well-known varieties of algebras defined by a 
family of polynomial identities of degree 3, such as Leibniz, Zinbiel, associative, Novikov and many other algebras. On the other hand, all algebras defined by the identities $(x y) z=0$ and $x(y z)=0$ are central extensions of some suitable algebra with zero product. The list of all non-anticommutative 4dimensional algebras defined by the identities $(x y) z=0$ and $x(y z)=0$ can be found in [8]. Note that there is only one 4-dimensional nilpotent anticommutative algebra satisfying the identity $(x y) z=0$. Obviously, all algebras from this list are 4-dimensional nilpotent $\mathfrak{C} \mathfrak{D}$-algebras.

1.2.2. 2-dimensional nilpotent algebras. There is only one non-zero 2-dimensional nilpotent algebra. It is a $\mathfrak{C} \mathfrak{D}$-algebra:

$$
\mathfrak{C} \mathfrak{D}_{01}^{2 *}: e_{1} e_{1}=e_{2}
$$

1.2.3. 3-dimensional nilpotent algebras. Thanks to [2], we have the classification of all 3dimensional nilpotent algebras. It is easy to see that each 3 -dimensional nilpotent algebra is a $\mathfrak{C} \mathfrak{D}$ algebra:

\begin{tabular}{lllll}
$\mathfrak{C} \mathfrak{D}_{01}^{3}$ & $:$ & $e_{1} e_{1}=e_{2}$ & $e_{2} e_{2}=e_{3}$ & \\
\hline $\mathfrak{C} \mathfrak{D}_{02}^{3}$ & $:$ & $e_{1} e_{1}=e_{2}$ & $e_{2} e_{1}=e_{3}$ & $e_{2} e_{2}=e_{3}$ \\
\hline $\mathfrak{C} \mathfrak{D}_{03}^{3}$ & $:$ & $e_{1} e_{1}=e_{2}$ & $e_{2} e_{1}=e_{3}$ & \\
\hline $\mathfrak{C D}_{04}^{3}(\lambda)$ & $:$ & $e_{1} e_{1}=e_{2}$ & $e_{1} e_{2}=e_{3}$ & $e_{2} e_{1}=\lambda e_{3}$ \\
\hline $\mathfrak{C} \mathfrak{D}_{01}^{3 *}$ & $:$ & $e_{1} e_{1}=e_{2}$ & & \\
\hline $\mathfrak{C} \mathfrak{D}_{02}^{3 *}$ & $:$ & $e_{1} e_{1}=e_{3}$ & $e_{2} e_{2}=e_{3}$ & \\
\hline $\mathfrak{C} \mathfrak{D}_{03}^{3 *}$ & $:$ & $e_{1} e_{2}=e_{3}$ & $e_{2} e_{1}=-e_{3}$ & \\
\hline $\mathfrak{C} \mathfrak{D}_{04}^{3 *}(\lambda)$ & $:$ & $e_{1} e_{1}=\lambda e_{3}$ & $e_{2} e_{1}=e_{3}$ & $e_{2} e_{2}=e_{3}$
\end{tabular}

1.2.4. 4-dimensional nilpotent algebras with 2-dimensional annihilator. Thanks to [2], we have the classification of all 4-dimensional non-split nilpotent non-trivial algebras with 2-dimensional annihilator. All of these algebras are $\mathfrak{C} \mathfrak{D}$-algebras:

\begin{tabular}{llllll}
$\mathfrak{C} \mathfrak{D}_{05}^{4}$ & $:$ & $e_{1} e_{1}=e_{2}$ & $e_{2} e_{1}=e_{4}$ & $e_{2} e_{2}=e_{3}$ & \\
\hline $\mathfrak{C} \mathfrak{D}_{06}^{4}$ & $:$ & $e_{1} e_{1}=e_{2}$ & $e_{1} e_{2}=e_{4}$ & $e_{2} e_{1}=e_{3}$ & \\
\hline $\mathfrak{C} \mathfrak{D}_{07}^{4}(\lambda)$ & $:$ & $e_{1} e_{1}=e_{2}$ & $e_{1} e_{2}=e_{4}$ & $e_{2} e_{1}=\lambda e_{4}$ & $e_{2} e_{2}=e_{3}$
\end{tabular}

1.2.5. 4-dimensional nilpotent algebras with 1-dimensional annihilator. Thanks to [14] we have the classification of all 4 -dimensional nilpotent $\mathfrak{C} \mathfrak{D}$-algebras with 1 -dimensional annihilator. The remaining nilpotent non-CD-algebras with 1-dimensional annihilator will be found in the present paper.

1.2.6. Trivial central extensions. Thanks to [14] we know that all central extensions of $\mathfrak{C} \mathfrak{D}_{01}^{3 *}, \mathfrak{C} \mathfrak{D}_{02}^{3 *}$, $\mathfrak{C} \mathfrak{D}_{03}^{3 *}$ and $\mathfrak{C} \mathfrak{D}_{04}^{3 *}$ are $\mathfrak{C} \mathfrak{D}$-algebras.

1.3. 1-dimensional central extensions of $\mathfrak{C} \mathfrak{D}_{01}^{3}$. Here we collect all information about $\mathfrak{C} \mathfrak{D}_{01}^{3}$ : 


\begin{tabular}{|l|l|ll|}
\hline Algebra & Multiplication & Cohomology & \\
\hline \multirow{2}{*}{$\mathfrak{C D}_{01}^{3}$} & $e_{1} e_{1}=e_{2}$ & $\mathrm{H}_{\mathfrak{C D}}^{2}\left(\mathfrak{C D}_{01}^{3}\right)=\left\langle\left[\Delta_{12}\right],\left[\Delta_{21}\right]\right\rangle$ \\
& $e_{2} e_{2}=e_{3}$ & $\mathrm{H}^{2}\left(\mathfrak{C} \mathfrak{D}_{01}^{3}\right)=\mathrm{H}_{\mathfrak{C D}}^{2}\left(\mathfrak{C} \mathfrak{D}_{01}^{3}\right) \oplus\left\langle\left[\Delta_{13}\right],\left[\Delta_{31}\right],\left[\Delta_{23}\right],\left[\Delta_{32}\right],\left[\Delta_{33}\right]\right\rangle$ \\
\hline
\end{tabular}

Let us use the following notations

$$
\begin{array}{llll}
\nabla_{1}=\left[\Delta_{12}\right] & \nabla_{2}=\left[\Delta_{21}\right] & \nabla_{3}=\left[\Delta_{13}\right] & \nabla_{4}=\left[\Delta_{31}\right] \\
\nabla_{5}=\left[\begin{array}{lll}
\left.\Delta_{23}\right] & \nabla_{6}=\left[\Delta_{32}\right] & \nabla_{7}=\left[\Delta_{33}\right.
\end{array}\right] .
\end{array}
$$

Take $\theta=\sum_{i=1}^{7} \alpha_{i} \nabla_{i} \in \mathrm{H}^{2}\left(\mathfrak{C} \mathfrak{D}_{01}^{3}\right)$. If

$$
\phi=\left(\begin{array}{ccc}
x & 0 & 0 \\
0 & x^{2} & 0 \\
y & 0 & x^{4}
\end{array}\right) \in \operatorname{Aut} \mathfrak{C} \mathfrak{D}_{01}^{3}
$$

then

where

$$
\phi^{T}\left(\begin{array}{ccc}
0 & \alpha_{1} & \alpha_{3} \\
\alpha_{2} & 0 & \alpha_{5} \\
\alpha_{4} & \alpha_{6} & \alpha_{7}
\end{array}\right) \phi=\left(\begin{array}{ccc}
\alpha^{*} & \alpha_{1}^{*} & \alpha_{3}^{*} \\
\alpha_{2}^{*} & 0 & \alpha_{5}^{*} \\
\alpha_{4}^{*} & \alpha_{6}^{*} & \alpha_{7}^{*}
\end{array}\right)
$$

$$
\begin{aligned}
& \alpha_{1}^{*}=x^{2}\left(x \alpha_{1}+y \alpha_{6}\right) \quad \alpha_{2}^{*}=x^{2}\left(x \alpha_{2}+y \alpha_{5}\right) \quad \alpha_{3}^{*}=x^{4}\left(x \alpha_{3}+y \alpha_{7}\right) \quad \alpha_{4}^{*}=x^{4}\left(x \alpha_{4}+y \alpha_{7}\right) \\
& \alpha_{5}^{*}=x^{6} \alpha_{5} \quad \alpha_{6}^{*}=x^{6} \alpha_{6} \quad \alpha_{7}^{*}=x^{8} \alpha_{7} .
\end{aligned}
$$

Hence, $\phi\langle\theta\rangle=\left\langle\theta^{*}\right\rangle$, where $\theta^{*}=\sum_{i=1}^{7} \alpha_{i}^{*} \nabla_{i}$. We are only interested in elements with $\left(\alpha_{3}, \alpha_{4}, \alpha_{5}, \alpha_{6}, \alpha_{7}\right) \neq(0,0,0,0,0)$. Then

(1) $\alpha_{7} \neq 0$.

(a) $\alpha_{6} \neq 0$. Then choosing $x=\sqrt{\alpha_{6} \alpha_{7}^{-1}}$ and $y=-\alpha_{4} \alpha_{7}^{-1} x$, we have the family of representatives $\left\langle\alpha \nabla_{1}+\beta \nabla_{2}+\gamma \nabla_{3}+\delta \nabla_{5}+\nabla_{6}+\nabla_{7}\right\rangle$. Observe that two distinct quadruples $(\alpha, \beta, \gamma, \delta)$ and $\left(\alpha^{\prime}, \beta^{\prime}, \gamma^{\prime}, \delta^{\prime}\right)$ determine the same orbit if and only if $(\alpha, \beta, \gamma, \delta)=\left(-\alpha^{\prime},-\beta^{\prime},-\gamma^{\prime}, \delta^{\prime}\right)$.

(b) $\alpha_{6}=0$ and $\alpha_{5} \neq 0$. Choosing $x=\sqrt{\alpha_{5} \alpha_{7}^{-1}}$ and $y=-\alpha_{4} \alpha_{7}^{-1} x$, we have the family of representatives $\left\langle\alpha \nabla_{1}+\beta \nabla_{2}+\gamma \nabla_{3}+\nabla_{5}+\nabla_{7}\right\rangle$. Observe that two distinct triples $(\alpha, \beta, \gamma)$ and $\left(\alpha^{\prime}, \beta^{\prime}, \gamma^{\prime}\right)$ determine the same orbit if and only if $(\alpha, \beta, \gamma)=\left(-\alpha^{\prime},-\beta^{\prime},-\gamma^{\prime}\right)$.

(c) $\alpha_{6}=\alpha_{5}=0$ and $\alpha_{3} \neq \alpha_{4}$. Choosing $x=\sqrt[3]{\left(\alpha_{3}-\alpha_{4}\right) \alpha_{7}^{-1}}$ and $y=-\alpha_{4} \alpha_{7}^{-1} x$, we have the family of representatives $\left\langle\alpha \nabla_{1}+\beta \nabla_{2}+\nabla_{3}+\nabla_{7}\right\rangle$. Observe that two pairs $(\alpha, \beta)$ and $\left(\alpha^{\prime}, \beta^{\prime}\right)$ determine the same orbit if and only if $(\alpha, \beta)=\left(\xi \alpha^{\prime}, \xi \beta^{\prime}\right)$, where $\xi^{3}=1$.

(d) $\alpha_{6}=\alpha_{5}=0, \alpha_{3}=\alpha_{4}$ and $\alpha_{2} \neq 0$. Choosing $x=\sqrt[5]{\alpha_{2} \alpha_{7}^{-1}}$ and $y=-\alpha_{4} \alpha_{7}^{-1} x$, we have the family of representatives of distinct orbits $\left\langle\alpha \nabla_{1}+\nabla_{2}+\nabla_{7}\right\rangle$.

(e) $\alpha_{6}=\alpha_{5}=0, \alpha_{3}=\alpha_{4}$ and $\alpha_{2}=0$. Choosing $y=-\alpha_{4} \alpha_{7}^{-1} x$, we have two representatives $\left\langle\nabla_{7}\right\rangle$ and $\left\langle\nabla_{1}+\nabla_{7}\right\rangle$ depending on whether $\alpha_{1}=0$ or not. 
(2) $\alpha_{7}=0$

(a) $\alpha_{6} \neq 0$ and $\alpha_{4} \neq 0$. Choosing $x=\alpha_{4} \alpha_{6}^{-1}$ and $y=-\alpha_{1} \alpha_{4} \alpha_{6}^{-2}$, we have the family of representatives of distinct orbits $\left\langle\alpha \nabla_{2}+\beta \nabla_{3}+\nabla_{4}+\gamma \nabla_{5}+\nabla_{6}\right\rangle$.

(b) $\alpha_{6} \neq 0, \alpha_{4}=0$ and $\alpha_{3} \neq 0$. Choosing $x=\alpha_{3} \alpha_{6}^{-1}$ and $y=-\alpha_{1} \alpha_{3} \alpha_{6}^{-2}$, we have the family of representatives of distinct orbits $\left\langle\alpha \nabla_{2}+\nabla_{3}+\beta \nabla_{5}+\nabla_{6}\right\rangle$.

(c) $\alpha_{6} \neq 0$ and $\alpha_{4}=\alpha_{3}=0$. Choosing $y=-\alpha_{1} \alpha_{6}^{-1} x$ we have two families of representatives of distinct orbits $\left\langle\alpha \nabla_{5}+\nabla_{6}\right\rangle$ and $\left\langle\nabla_{2}+\alpha \nabla_{5}+\nabla_{6}\right\rangle$ depending on whether $\alpha_{1} \alpha_{5}-\alpha_{2} \alpha_{6}=0$ or not.

(d) $\alpha_{6}=0, \alpha_{5} \neq 0$ and $\alpha_{4} \neq 0$. Choosing $x=\alpha_{4} \alpha_{5}^{-1}$ and $y=-\alpha_{2} \alpha_{4} \alpha_{5}^{-2}$, we have the family of representatives of distinct orbits $\left\langle\alpha \nabla_{1}+\beta \nabla_{3}+\nabla_{4}+\nabla_{5}\right\rangle$.

(e) $\alpha_{6}=0, \alpha_{5} \neq 0$ and $\alpha_{4}=0$. Choosing $y=-\alpha_{2} \alpha_{5}^{-1} x$, we have the following representatives of distinct orbits $\left\langle\alpha \nabla_{1}+\nabla_{3}+\nabla_{5}\right\rangle,\left\langle\nabla_{1}+\nabla_{5}\right\rangle$ and $\left\langle\nabla_{5}\right\rangle$, corresponding to the 3 cases: $\alpha_{3} \neq 0 ; \alpha_{3}=0, \alpha_{1} \neq 0 ; \alpha_{3}=\alpha_{1}=0$, respectively.

(f) $\alpha_{6}=\alpha_{5}=0$ and $\alpha_{4} \neq 0$. We have the following families of representatives of distinct orbits $\left\langle\nabla_{1}+\alpha \nabla_{2}+\beta \nabla_{3}+\nabla_{4}\right\rangle,\left\langle\nabla_{2}+\alpha \nabla_{3}+\nabla_{4}\right\rangle$ and $\left\langle\alpha \nabla_{3}+\nabla_{4}\right\rangle$ corresponding to the 3 cases: $\alpha_{1} \neq 0 ; \alpha_{1}=0, \alpha_{2} \neq 0 ; \alpha_{1}=\alpha_{2}=0$, respectively.

(g) $\alpha_{6}=\alpha_{5}=\alpha_{4}=0$ and $\alpha_{3} \neq 0$. We have the following representatives of distinct orbits $\left\langle\alpha \nabla_{1}+\nabla_{2}+\nabla_{3}\right\rangle,\left\langle\nabla_{1}+\nabla_{3}\right\rangle$ and $\left\langle\nabla_{3}\right\rangle$ corresponding to the 3 cases: $\alpha_{2} \neq 0 ; \alpha_{2}=0$, $\alpha_{1} \neq 0 ; \alpha_{2}=\alpha_{1}=0$, respectively.

Summarizing, we have the following distinct orbits:

$$
\begin{aligned}
& \left\langle\alpha \nabla_{1}+\nabla_{2}+\nabla_{3}\right\rangle \quad\left\langle\alpha \nabla_{1}+\beta \nabla_{2}+\gamma \nabla_{3}+\delta \nabla_{5}+\nabla_{6}+\nabla_{7}\right\rangle^{O(\alpha, \beta, \gamma, \delta)=O(-\alpha,-\beta,-\gamma, \delta)} \\
& \left\langle\alpha \nabla_{1}+\nabla_{3}+\nabla_{5}\right\rangle \quad\left\langle\alpha \nabla_{1}+\beta \nabla_{2}+\gamma \nabla_{3}+\nabla_{5}+\nabla_{7}\right\rangle^{O(\alpha, \beta, \gamma)=O(-\alpha,-\beta,-\gamma)} \\
& \left\langle\alpha \nabla_{1}+\beta \nabla_{2}+\nabla_{3}+\nabla_{7}\right\rangle^{O(\alpha, \beta)=O(\sqrt[3]{1}(\alpha, \beta))} \quad\left\langle\alpha \nabla_{1}+\nabla_{2}+\nabla_{7}\right\rangle \\
& \left\langle\nabla_{1}+\nabla_{3}\right\rangle \quad\left\langle\nabla_{1}+\alpha \nabla_{2}+\beta \nabla_{3}+\nabla_{4}\right\rangle \quad\left\langle\alpha \nabla_{1}+\beta \nabla_{3}+\nabla_{4}+\nabla_{5}\right\rangle \\
& \left\langle\nabla_{1}+\nabla_{5}\right\rangle \quad\left\langle\nabla_{1}+\nabla_{7}\right\rangle \\
& \left\langle\nabla_{2}+\alpha \nabla_{3}+\nabla_{4}\right\rangle \\
& \left\langle\alpha \nabla_{2}+\beta \nabla_{3}+\nabla_{4}+\gamma \nabla_{5}+\nabla_{6}\right\rangle \quad\left\langle\alpha \nabla_{2}+\nabla_{3}+\beta \nabla_{5}+\nabla_{6}\right\rangle \quad\left\langle\nabla_{2}+\alpha \nabla_{5}+\nabla_{6}\right\rangle \\
& \left\langle\alpha \nabla_{3}+\nabla_{4}\right\rangle \quad\left\langle\nabla_{3}\right\rangle \\
& \left\langle\alpha \nabla_{5}+\nabla_{6}\right\rangle \quad\left\langle\nabla_{7}\right\rangle
\end{aligned}
$$

They correspond to the following new algebras:

\begin{tabular}{llllllll}
$\mathbf{N}_{01}^{4}(\alpha)$ & $:$ & $e_{1} e_{1}=e_{2}$ & $e_{1} e_{2}=\alpha e_{4}$ & $e_{1} e_{3}=e_{4}$ & $e_{2} e_{1}=e_{4}$ & $e_{2} e_{2}=e_{3}$ & \\
\hline $\mathbf{N}_{02}^{4}(\alpha, \beta, \gamma, \delta)$ & $:$ & $e_{1} e_{1}=e_{2}$ & $e_{1} e_{2}=\alpha e_{4}$ & $e_{1} e_{3}=\gamma e_{4}$ & $e_{2} e_{1}=\beta e_{4}$ & & \\
& & $e_{2} e_{2}=e_{3}$ & $e_{2} e_{3}=\delta e_{4}$ & $e_{3} e_{2}=e_{4}$ & $e_{3} e_{3}=e_{4}$ & \\
\hline $\mathbf{N}_{03}^{4}(\alpha)$ & $:$ & $e_{1} e_{1}=e_{2}$ & $e_{1} e_{2}=\alpha e_{4}$ & $e_{1} e_{3}=e_{4}$ & $e_{2} e_{2}=e_{3}$ & $e_{2} e_{3}=e_{4}$ & \\
\hline $\mathbf{N}_{04}^{4}(\alpha, \beta, \gamma)$ & $:$ & $e_{1} e_{1}=e_{2}$ & $e_{1} e_{2}=\alpha e_{4}$ & $e_{1} e_{3}=\gamma e_{4}$ & $e_{2} e_{1}=\beta e_{4}$ & & \\
& & $e_{2} e_{2}=e_{3}$ & $e_{2} e_{3}=e_{4}$ & $e_{3} e_{3}=e_{4}$ & & & \\
\hline $\mathbf{N}_{05}^{4}(\alpha, \beta)$ & $:$ & $e_{1} e_{1}=e_{2}$ & $e_{1} e_{2}=\alpha e_{4}$ & $e_{1} e_{3}=e_{4}$ & $e_{2} e_{1}=\beta e_{4}$ & $e_{2} e_{2}=e_{3}$ & $e_{3} e_{3}=e_{4}$ \\
\hline $\mathbf{N}_{06}^{4}(\alpha)$ & $:$ & $e_{1} e_{1}=e_{2}$ & $e_{1} e_{2}=\alpha e_{4}$ & $e_{2} e_{1}=e_{4}$ & $e_{2} e_{2}=e_{3}$ & $e_{3} e_{3}=e_{4}$ & \\
\hline $\mathbf{N}_{07}^{4}$ & $:$ & $e_{1} e_{1}=e_{2}$ & $e_{1} e_{2}=e_{4}$ & $e_{1} e_{3}=e_{4}$ & $e_{2} e_{2}=e_{3}$ & & \\
\hline $\mathbf{N}_{08}^{4}(\alpha, \beta)$ & $:$ & $e_{1} e_{1}=e_{2}$ & $e_{1} e_{2}=e_{4}$ & $e_{1} e_{3}=\beta e_{4}$ & $e_{2} e_{1}=\alpha e_{4}$ & $e_{2} e_{2}=e_{3}$ & $e_{3} e_{1}=e_{4}$ \\
\hline $\mathbf{N}_{09}^{4}(\alpha, \beta)$ & $:$ & $e_{1} e_{1}=e_{2}$ & $e_{1} e_{2}=\alpha e_{4}$ & $e_{1} e_{3}=\beta e_{4}$ & $e_{2} e_{2}=e_{3}$ & $e_{2} e_{3}=e_{4}$ & $e_{3} e_{1}=e_{4}$ \\
\hline $\mathbf{N}_{10}^{4}$ & $:$ & $e_{1} e_{1}=e_{2}$ & $e_{1} e_{2}=e_{4}$ & $e_{2} e_{2}=e_{3}$ & $e_{2} e_{3}=e_{4}$ & & \\
\hline & & & & & & & \\
\hline
\end{tabular}




\begin{tabular}{|c|c|c|c|c|c|c|c|}
\hline $\mathbf{N}_{11}^{4}$ & $:$ & $e_{1} e_{1}=e_{2}$ & $e_{1} e_{2}=e_{4}$ & $e_{2} e_{2}=e_{3}$ & $e_{3} e_{3}=e_{4}$ & & \\
\hline $\mathbf{N}_{12}^{4}(\alpha)$ & $:$ & $e_{1} e_{1}=e_{2}$ & $e_{1} e_{3}=\alpha e_{4}$ & $e_{2} e_{1}=e_{4}$ & $e_{2} e_{2}=e_{3}$ & $e_{3} e_{1}=e_{4}$ & \\
\hline $\mathbf{N}_{13}^{4}(\alpha, \beta, \gamma)$ & $\overline{:}$ & $e_{1} e_{1}=e_{2}$ & $e_{1} e_{3}=\beta e_{4}$ & $e_{2} e_{1}=\alpha e_{4}$ & $e_{2} e_{2}=e_{3}$ & & \\
\hline & & $e_{2} e_{3}=\gamma e_{4}$ & $e_{3} e_{1}=e_{4}$ & $e_{3} e_{2}=e_{4}$ & & & \\
\hline $\mathbf{N}_{14}^{4}(\alpha, \beta)$ & $:$ & $e_{1} e_{1}=e_{2}$ & $e_{1} e_{3}=e_{4}$ & $e_{2} e_{1}=\alpha e_{4}$ & $e_{2} e_{2}=e_{3}$ & $e_{2} e_{3}=\beta e_{4}$ & $e_{3} e_{2}=e_{4}$ \\
\hline $\mathbf{N}_{15}^{4}(\alpha)$ & $:$ & $e_{1} e_{1}=e_{2}$ & $e_{2} e_{1}=e_{4}$ & $e_{2} e_{2}=e_{3}$ & $e_{2} e_{3}=\alpha e_{4}$ & $e_{3} e_{2}=e_{4}$ & \\
\hline $\mathbf{N}_{16}^{4}(\alpha)$ & $:$ & $e_{1} e_{1}=e_{2}$ & $e_{1} e_{3}=\alpha e_{4}$ & $e_{2} e_{2}=e_{3}$ & $e_{3} e_{1}=e_{4}$ & & \\
\hline $\mathbf{N}_{17}^{4}$ & $:$ & $e_{1} e_{1}=e_{2}$ & $e_{1} e_{3}=e_{4}$ & $e_{2} e_{2}=e_{3}$ & & & \\
\hline $\mathbf{N}_{18}^{4}$ & $:$ & $e_{1} e_{1}=e_{2}$ & $e_{2} e_{2}=e_{3}$ & $e_{2} e_{3}=e_{4}$ & & & \\
\hline $\mathbf{N}_{19}^{4}(\alpha)$ & $:$ & $e_{1} e_{1}=e_{2}$ & $e_{2} e_{2}=e_{3}$ & $e_{2} e_{3}=\alpha e_{4}$ & $e_{3} e_{2}=e_{4}$ & & \\
\hline $\mathbf{N}_{20}^{4}$ & $:$ & $e_{1} e_{1}=e_{2}$ & $e_{2} e_{2}=e_{3}$ & $e_{3} e_{3}=e_{4}$ & & & \\
\hline
\end{tabular}

$$
\begin{gathered}
\mathbf{N}_{02}^{4}(\alpha, \beta, \gamma, \delta) \cong \mathbf{N}_{02}^{4}(-(\alpha, \beta, \gamma), \delta) \quad \mathbf{N}_{04}^{4}(\alpha, \beta, \gamma) \cong \mathbf{N}_{04}^{4}(-(\alpha, \beta, \gamma)) \\
\mathbf{N}_{05}^{4}(\alpha, \beta) \cong \mathbf{N}_{05}^{4}(\sqrt[3]{1}(\alpha, \beta))
\end{gathered}
$$

1.4. 1-dimensional central extensions of $\mathfrak{C} \mathfrak{D}_{02}^{3}$. Here we collect all information about $\mathfrak{C} \mathfrak{D}_{02}^{3}$ :

\begin{tabular}{|l|l|ll|}
\hline Algebra & Multiplication & Cohomology & \\
\hline & $e_{1} e_{1}=e_{2}$ & $\mathrm{H}_{\mathfrak{C D}}^{2}\left(\mathfrak{C} \mathfrak{D}_{02}^{3}\right)=\left\langle\left[\Delta_{12}\right],\left[\Delta_{21}\right]\right\rangle$ \\
$\mathfrak{C} \mathfrak{D}_{02}^{3}$ & $e_{2} e_{1}=e_{3}$ & $\mathrm{H}^{2}\left(\mathfrak{C} \mathfrak{D}_{02}^{3}\right)=\mathrm{H}_{\mathfrak{C D}}^{2}\left(\mathfrak{C} \mathfrak{D}_{02}^{3}\right) \oplus\left\langle\left[\Delta_{13}\right],\left[\Delta_{31}\right],\left[\Delta_{23}\right],\left[\Delta_{32}\right],\left[\Delta_{33}\right]\right\rangle$ \\
& $e_{2} e_{2}=e_{3}$ & \\
\hline
\end{tabular}

Let us use the following notations

$$
\begin{aligned}
& \nabla_{1}=\left[\Delta_{12}\right] \quad \nabla_{2}=\left[\Delta_{21}\right] \quad \nabla_{3}=\left[\Delta_{13}\right] \quad \nabla_{4}=\left[\Delta_{31}\right] \\
& \nabla_{5}=\left[\Delta_{23}\right] \quad \nabla_{6}=\left[\Delta_{32}\right] \quad \nabla_{7}=\left[\Delta_{33}\right] \text {. }
\end{aligned}
$$

Take $\theta=\sum_{i=1}^{7} \alpha_{i} \nabla_{i} \in \mathrm{H}^{2}\left(\mathfrak{C} \mathfrak{D}_{02}^{3}\right)$. If

$$
\phi=\left(\begin{array}{lll}
1 & 0 & 0 \\
0 & 1 & 0 \\
x & 0 & 1
\end{array}\right) \in \text { Aut } \mathfrak{C D}_{02}^{3},
$$

then

where

$$
\phi^{T}\left(\begin{array}{ccc}
0 & \alpha_{1} & \alpha_{3} \\
\alpha_{2} & 0 & \alpha_{5} \\
\alpha_{4} & \alpha_{6} & \alpha_{7}
\end{array}\right) \phi=\left(\begin{array}{ccc}
\alpha^{*} & \alpha_{1}^{*} & \alpha_{3}^{*} \\
\alpha_{2}^{*} & 0 & \alpha_{5}^{*} \\
\alpha_{4}^{*} & \alpha_{6}^{*} & \alpha_{7}^{*}
\end{array}\right)
$$

$$
\begin{array}{llll}
\alpha_{1}^{*}=\alpha_{1}+x \alpha_{6} & \alpha_{2}^{*}=\alpha_{2}+x \alpha_{5} & \alpha_{3}^{*}=\alpha_{3}+x \alpha_{7} & \alpha_{4}^{*}=\alpha_{4}+x \alpha_{7} \\
\alpha_{5}^{*}=\alpha_{5} & \alpha_{6}^{*}=\alpha_{6} & \alpha_{7}^{*}=\alpha_{7} .
\end{array}
$$

Hence, $\phi\langle\theta\rangle=\left\langle\theta^{*}\right\rangle$, where $\theta^{*}=\sum_{i=1}^{7} \alpha_{i}^{*} \nabla_{i}$. We are only interested in elements with $\left(\alpha_{3}, \alpha_{4}, \alpha_{5}, \alpha_{6}, \alpha_{7}\right) \neq(0,0,0,0,0)$. Then 
(1) $\alpha_{7} \neq 0$. Then choosing $x=-\alpha_{3} \alpha_{7}^{-1}$, we obtain the family of representatives of distinct orbits $\left\langle\alpha \nabla_{1}+\beta \nabla_{2}+\gamma \nabla_{4}+\delta \nabla_{5}+\varepsilon \nabla_{6}+\nabla_{7}\right\rangle$.

(2) $\alpha_{7}=0$ and $\alpha_{6} \neq 0$. Then choosing $x=-\alpha_{1} \alpha_{6}^{-1}$, we obtain the family of representatives of distinct orbits $\left\langle\alpha \nabla_{2}+\beta \nabla_{3}+\gamma \nabla_{4}+\delta \nabla_{5}+\nabla_{6}\right\rangle$.

(3) $\alpha_{7}=\alpha_{6}=0$ and $\alpha_{5} \neq 0$. Then choosing $x=-\alpha_{2} \alpha_{5}^{-1}$, we obtain the family of representatives of distinct orbits $\left\langle\alpha \nabla_{1}+\beta \nabla_{3}+\gamma \nabla_{4}+\nabla_{5}\right\rangle$.

(4) $\alpha_{7}=\alpha_{6}=\alpha_{5}=0$ and $\alpha_{4} \neq 0$. Then we obtain the family of representatives of distinct orbits $\left\langle\alpha \nabla_{1}+\beta \nabla_{2}+\gamma \nabla_{3}+\nabla_{4}\right\rangle$.

(5) $\alpha_{7}=\alpha_{6}=\alpha_{5}=\alpha_{4}=0$ and $\alpha_{3} \neq 0$. Then we obtain the family of representatives of distinct orbits $\left\langle\alpha \nabla_{1}+\beta \nabla_{2}+\nabla_{3}\right\rangle$.

Summarizing, we have the following distinct orbits:

$$
\begin{array}{cl}
\left\langle\alpha \nabla_{1}+\beta \nabla_{2}+\nabla_{3}\right\rangle & \left\langle\alpha \nabla_{1}+\beta \nabla_{2}+\gamma \nabla_{3}+\nabla_{4}\right\rangle \\
\left\langle\alpha \nabla_{1}+\beta \nabla_{3}+\gamma \nabla_{4}+\nabla_{5}\right\rangle & \left\langle\alpha \nabla_{2}+\beta \nabla_{3}+\gamma \nabla_{4}+\delta \nabla_{5}+\nabla_{6}\right\rangle \\
\left\langle\alpha \nabla_{1}+\beta \nabla_{2}+\gamma \nabla_{4}+\delta \nabla_{5}+\varepsilon \nabla_{6}+\nabla_{7}\right\rangle
\end{array}
$$

\begin{tabular}{|c|c|c|c|c|c|c|c|}
\hline $\mathbf{N}_{21}^{4}(\alpha, \beta)$ & $:$ & $e_{1} e_{1}=e_{2}$ & $e_{1} e_{2}=\alpha e_{4}$ & $e_{1} e_{3}=e_{4}$ & $e_{2} e_{1}=e_{3}+\beta e_{4}$ & $e_{2} e_{2}=e_{3}$ & \\
\hline $\mathbf{N}_{22}^{4}(\alpha, \beta, \gamma)$ & $:$ & $e_{1} e_{1}=e_{2}$ & $e_{1} e_{2}=\alpha e_{4}$ & $e_{1} e_{3}=\gamma e_{4}$ & $e_{2} e_{1}=e_{3}+\beta e_{4}$ & $e_{2} e_{2}=e_{3}$ & $e_{3} e_{1}=e_{4}$ \\
\hline $\mathbf{N}_{23}^{4}(\alpha, \beta, \gamma)$ & : & $e_{1} e_{1}=e_{2}$ & $\overline{e_{1} e_{2}=\alpha e_{4}}$ & $e_{1} e_{3}=\beta e_{4}$ & $e_{2} e_{1}=e_{3}$ & & \\
\hline & & $e_{2} e_{2}=e_{3}$ & $e_{2} e_{3}=e_{4}$ & $e_{3} e_{1}=\gamma e_{4}$ & & & \\
\hline $\mathbf{N}_{24}^{4}(\alpha, \beta, \gamma, \delta)$ & : & $\begin{array}{l}e_{1} e_{1}=e_{2} \\
e_{2} e_{3}=\delta e_{4}\end{array}$ & $\begin{array}{l}e_{1} e_{3}=\beta e_{4} \\
e_{3} e_{1}=\gamma e_{4}\end{array}$ & $\begin{array}{l}e_{2} e_{1}=e_{3}+\alpha e_{4} \\
e_{3} e_{2}=e_{4}\end{array}$ & $e_{2} e_{2}=e_{3}$ & & \\
\hline $\mathbf{N}_{25}^{4}(\alpha, \beta, \gamma, \delta, \varepsilon)$ & : & $\begin{array}{l}e_{1} e_{1}=e_{2} \\
e_{2} e_{3}=\delta e_{4}\end{array}$ & $\begin{array}{l}e_{1} e_{2}=\alpha e_{4} \\
e_{3} e_{1}=\gamma e_{4}\end{array}$ & $\begin{array}{l}e_{2} e_{1}=e_{3}+\beta e_{4} \\
e_{3} e_{2}=\varepsilon e_{4}\end{array}$ & $\begin{array}{l}e_{2} e_{2}=e_{3} \\
e_{3} e_{3}=e_{4}\end{array}$ & & \\
\hline
\end{tabular}

This gives the following new algebras:

1.5. 1-dimensional central extensions of $\mathfrak{C} \mathfrak{D}_{03}^{3}$. Here we collect all information about $\mathfrak{C} \mathfrak{D}_{03}^{3}$ :

\begin{tabular}{|l|l|lll|}
\hline Algebra & Multiplication & Cohomology & \\
\hline \multirow{2}{*}{$\mathfrak{D}_{03}^{3}$} & $e_{1} e_{1}=e_{2}$ & $\mathrm{H}_{\mathfrak{C D}}^{2}\left(\mathfrak{C D}_{03}^{3}\right)=\left\langle\left[\Delta_{12}\right],\left[\Delta_{22}\right],\left[\Delta_{13}\right]-2\left[\Delta_{31}\right]\right\rangle$ \\
& $e_{2} e_{1}=e_{3}$ & $\mathrm{H}^{2}\left(\mathfrak{C} \mathfrak{D}_{03}^{3}\right)=\mathrm{H}_{\mathfrak{C D}}^{2}\left(\mathfrak{C} \mathfrak{D}_{03}^{3}\right) \oplus\left\langle\left[\Delta_{31}\right],\left[\Delta_{23}\right],\left[\Delta_{32}\right],\left[\Delta_{33}\right]\right\rangle$ \\
\hline
\end{tabular}

Let us use the following notations

$$
\begin{array}{llll}
\nabla_{1}=\left[\Delta_{12}\right] & \nabla_{2}=\left[\Delta_{22}\right] & \nabla_{3}=\left[\Delta_{13}\right]-2\left[\Delta_{31}\right] & \nabla_{4}=\left[\Delta_{31}\right] \\
\nabla_{5}=\left[\Delta_{23}\right] & \nabla_{6}=\left[\Delta_{32}\right] & \nabla_{7}=\left[\Delta_{33}\right] .
\end{array}
$$

Take $\theta=\sum_{i=1}^{7} \alpha_{i} \nabla_{i} \in \mathrm{H}^{2}\left(\mathfrak{C} \mathfrak{D}_{03}^{3}\right)$. If

$$
\phi=\left(\begin{array}{ccc}
x & 0 & 0 \\
y & x^{2} & 0 \\
z & x y & x^{3}
\end{array}\right) \in \operatorname{Aut} \mathfrak{C D}_{03}^{3}
$$


then

where

$$
\phi^{T}\left(\begin{array}{ccc}
0 & \alpha_{1} & \alpha_{3} \\
0 & \alpha_{2} & \alpha_{5} \\
\alpha_{4}-2 \alpha_{3} & \alpha_{6} & \alpha_{7}
\end{array}\right) \phi=\left(\begin{array}{ccc}
\alpha^{*} & \alpha_{1}^{*} & \alpha_{3}^{*} \\
\alpha^{* *} & \alpha_{2}^{*} & \alpha_{5}^{*} \\
\alpha_{4}^{*}-2 \alpha_{3}^{*} & \alpha_{6}^{*} & \alpha_{7}^{*}
\end{array}\right)
$$

$$
\begin{aligned}
\alpha_{1}^{*} & =\left(\alpha_{1} x^{2}+\left(\alpha_{2}+\alpha_{3}\right) x y+\alpha_{5} y^{2}+\alpha_{6} x z+\alpha_{7} y z\right) x \\
\alpha_{2}^{*} & =\left(\alpha_{2} x^{2}+\left(\alpha_{5}+\alpha_{6}\right) x y+\alpha_{7} y^{2}\right) x^{2} \\
\alpha_{3}^{*} & =\left(\alpha_{3} x+\alpha_{5} y+\alpha_{7} z\right) x^{3} \\
\alpha_{4}^{*} & =\left(\alpha_{4} x+\left(2 \alpha_{5}+\alpha_{6}\right) y+3 \alpha_{7} z\right) x^{3} \\
\alpha_{5}^{*} & =\left(\alpha_{5} x+\alpha_{7} y\right) x^{4} \\
\alpha_{6}^{*} & =\left(\alpha_{6} x+\alpha_{7} y\right) x^{4} \\
\alpha_{7}^{*} & =\alpha_{7} x^{6}
\end{aligned}
$$

Hence, $\phi\langle\theta\rangle=\left\langle\theta^{*}\right\rangle$, where $\theta^{*}=\sum_{i=1}^{7} \alpha_{i}^{*} \nabla_{i}$. We are only interested in $\theta$ with $\left(\alpha_{4}, \alpha_{5}, \alpha_{6}, \alpha_{7}\right) \neq$ $(0,0,0,0)$.

(1) $\alpha_{7} \neq 0$. Then put $y=-\frac{\alpha_{6} x}{\alpha_{7}}$ and $z=\frac{x}{\alpha_{7}^{2}}\left(\alpha_{5} \alpha_{6}-\alpha_{3} \alpha_{7}\right)$ to make $\alpha_{3}^{*}=\alpha_{6}^{*}=0$.

(a) $\alpha_{5} \neq \alpha_{6}$. Then choosing $x=\frac{\alpha_{5}-\alpha_{6}}{\alpha_{7}}$, we obtain the family of representatives of distinct orbits $\left\langle\alpha \nabla_{1}+\beta \nabla_{2}+\gamma \nabla_{4}+\nabla_{5}+\nabla_{7}\right\rangle$.

(b) $\alpha_{5}=\alpha_{6}$ and $\alpha_{4} \neq 3 \alpha_{3}$. Then choosing $x=\sqrt{\frac{\alpha_{4}-3 \alpha_{3}}{3 \alpha_{7}}}$, we obtain the family of representatives $\left\langle\alpha \nabla_{1}+\beta \nabla_{2}+\nabla_{4}+\nabla_{7}\right\rangle$, where two distinct pairs $(\alpha, \beta)$ and $\left(\alpha^{\prime}, \beta^{\prime}\right)$ determine the same orbit if and only if $(\alpha, \beta)=\left(-\alpha^{\prime}, \beta^{\prime}\right)$.

(c) $\alpha_{5}=\alpha_{6}, \alpha_{4}=3 \alpha_{3}$ and $\alpha_{6}^{2} \neq \alpha_{2} \alpha_{7}$. Then choosing $x=\frac{1}{\alpha_{7}} \sqrt{\alpha_{2} \alpha_{7}-\alpha_{6}^{2}}$, we obtain the family of representatives $\left\langle\alpha \nabla_{1}+\nabla_{2}+\nabla_{7}\right\rangle$, where two distinct parameters $\alpha$ and $\alpha^{\prime}$ determine the same orbit if and only if $\alpha=-\alpha^{\prime}$.

(d) $\alpha_{5}=\alpha_{6}, \alpha_{4}=3 \alpha_{3}$ and $\alpha_{6}^{2}=\alpha_{2} \alpha_{7}$. Then we obtain 2 representatives $\left\langle\nabla_{7}\right\rangle$ and $\left\langle\nabla_{1}+\nabla_{7}\right\rangle$ depending on whether $\alpha_{3} \alpha_{6}=\alpha_{1} \alpha_{7}$ or not.

(2) $\alpha_{7}=0, \alpha_{5} \neq 0$ and $\alpha_{6} \neq 0$. Then $\alpha_{7}^{*}=0$, and we put $y=-\frac{\alpha_{3} x}{\alpha_{5}}$ and $z=\frac{x}{\alpha_{5} \alpha_{6}}\left(\alpha_{2} \alpha_{3}-\alpha_{1} \alpha_{5}\right)$ to make $\alpha_{1}^{*}=\alpha_{3}^{*}=0$.

(a) $\left(\alpha_{4}-2 \alpha_{3}\right) \alpha_{5} \neq \alpha_{3} \alpha_{6}$. Then choosing $x=\frac{1}{\alpha_{5} \alpha_{6}}\left(\left(\alpha_{4}-2 \alpha_{3}\right) \alpha_{5}-\alpha_{3} \alpha_{6}\right)$, we obtain the family of representatives of distinct orbits $\left\langle\alpha \nabla_{2}+\nabla_{4}+\beta \nabla_{5}+\nabla_{6}\right\rangle_{\beta \neq 0}$.

(b) $\left(\alpha_{4}-2 \alpha_{3}\right) \alpha_{5}=\alpha_{3} \alpha_{6}$ and $\left(\alpha_{2}-\alpha_{3}\right) \alpha_{5} \neq \alpha_{3} \alpha_{6}$. Then choosing $x=\frac{1}{\alpha_{5} \alpha_{6}}\left(\left(\alpha_{2}-\alpha_{3}\right) \alpha_{5}-\right.$ $\left.\alpha_{3} \alpha_{6}\right)$, we obtain the family of representatives of distinct orbits $\left\langle\nabla_{2}+\alpha \nabla_{5}+\nabla_{6}\right\rangle_{\alpha \neq 0}$.

(c) $\left(\alpha_{4}-2 \alpha_{3}\right) \alpha_{5}=\alpha_{3} \alpha_{6}=\left(\alpha_{2}-\alpha_{3}\right) \alpha_{5}$. Then we obtain the family of representatives of distinct orbits $\left\langle\alpha \nabla_{5}+\nabla_{6}\right\rangle_{\alpha \neq 0}$.

(3) $\alpha_{7}=\alpha_{6}=0$ and $\alpha_{5} \neq 0$. Then $\alpha_{6}^{*}=\alpha_{7}^{*}=0$, and we put $y=-\frac{\alpha_{3} x}{\alpha_{5}}$ to make $\alpha_{3}^{*}=0$.

(a) $\alpha_{4} \neq 2 \alpha_{3}$. Then choosing $x=\frac{\alpha_{4}-2 \alpha_{3}}{\alpha_{5}}$, we obtain the family of representatives of distinct orbits $\left\langle\alpha \nabla_{1}+\beta \nabla_{2}+\nabla_{4}+\nabla_{5}\right\rangle$. 
(b) $\alpha_{4}=2 \alpha_{3}$ and $\alpha_{2} \neq \alpha_{3}$. Then choosing $x=\frac{\alpha_{2}-\alpha_{3}}{\alpha_{5}}$, we obtain the family of representatives of distinct orbits $\left\langle\alpha \nabla_{1}+\nabla_{2}+\nabla_{5}\right\rangle$.

(c) $\alpha_{4}=2 \alpha_{3}$ and $\alpha_{2}=\alpha_{3}$. Then we obtain 2 representatives $\left\langle\nabla_{5}\right\rangle$ and $\left\langle\nabla_{1}+\nabla_{5}\right\rangle$ depending on whether $\alpha_{3}^{2}=\alpha_{1} \alpha_{5}$ or not.

(4) $\alpha_{7}=\alpha_{5}=0$ and $\alpha_{6} \neq 0$. Then $\alpha_{5}^{*}=\alpha_{7}^{*}=0$, and we put $y=-\frac{\alpha_{4} x}{\alpha_{5}}$ and $z=\frac{x}{\alpha_{6}^{2}}\left(\left(\alpha_{2}+\right.\right.$ $\left.\left.\alpha_{3}\right) \alpha_{4}-\alpha_{1} \alpha_{6}\right)$ to make $\alpha_{1}^{*}=\alpha_{4}^{*}=0$.

(a) $\alpha_{2} \neq \alpha_{4}$. Then choosing $x=\frac{\alpha_{2}-\alpha_{4}}{\alpha_{6}}$, we obtain the family of representatives of distinct orbits $\left\langle\nabla_{2}+\alpha \nabla_{3}+\nabla_{6}\right\rangle$.

(b) $\alpha_{2}=\alpha_{4}$. Then we obtain 2 representatives $\left\langle\nabla_{6}\right\rangle$ and $\left\langle\nabla_{3}+\nabla_{6}\right\rangle$ depending on whether $\alpha_{3}=0$ or not. We will join $\left\langle\nabla_{6}\right\rangle$ with the family $\left\langle\alpha \nabla_{5}+\nabla_{6}\right\rangle_{\alpha \neq 0}$ found above.

(5) $\alpha_{7}=\alpha_{6}=\alpha_{5}=0$ and $\alpha_{4} \neq 0$. Then $\alpha_{5}^{*}=\alpha_{6}^{*}=\alpha_{7}^{*}=0$.

(a) $\alpha_{2} \neq-\alpha_{3}$. Then choosing $y=-\frac{\alpha_{1} x}{\alpha_{2}+\alpha_{3}}$, we obtain the family of representatives of distinct orbits $\left\langle\alpha \nabla_{2}+\beta \nabla_{3}+\nabla_{4}\right\rangle_{\beta \neq-\alpha}$.

(b) $\alpha_{2}=-\alpha_{3}$. Then we obtain two families of representatives of distinct orbits $\left\langle\alpha \nabla_{2}-\right.$ $\left.\alpha \nabla_{3}+\nabla_{4}\right\rangle$ and $\left\langle\nabla_{1}+\alpha \nabla_{2}-\alpha \nabla_{3}+\nabla_{4}\right\rangle$ depending on whether $\alpha_{1}=0$ or not. The family $\left\langle\alpha \nabla_{2}-\alpha \nabla_{3}+\nabla_{4}\right\rangle$ will be joined with the family $\left\langle\alpha \nabla_{2}+\beta \nabla_{3}+\nabla_{4}\right\rangle_{\beta \neq-\alpha}$ from the previous item.

Summarizing, we have the following distinct orbits:

$$
\begin{array}{lll}
\left\langle\nabla_{1}+\alpha \nabla_{2}-\alpha \nabla_{3}+\nabla_{4}\right\rangle & \left\langle\alpha \nabla_{1}+\beta \nabla_{2}+\nabla_{4}+\nabla_{5}\right\rangle & \left\langle\alpha \nabla_{1}+\beta \nabla_{2}+\gamma \nabla_{4}+\nabla_{5}+\nabla_{7}\right\rangle \\
\left\langle\alpha \nabla_{1}+\beta \nabla_{2}+\nabla_{4}+\nabla_{7}\right\rangle & O(\alpha, \beta)=O(-\alpha, \beta) & \left\langle\alpha \nabla_{1}+\nabla_{2}+\nabla_{5}\right\rangle \\
\left\langle\alpha \nabla_{1}+\nabla_{2}+\nabla_{7}\right\rangle^{O(\alpha)=O(-\alpha)} & \left\langle\nabla_{1}+\nabla_{5}\right\rangle & \left\langle\nabla_{1}+\nabla_{7}\right\rangle \\
\left\langle\alpha \nabla_{2}+\beta \nabla_{3}+\nabla_{4}\right\rangle & \left\langle\nabla_{2}+\alpha \nabla_{3}+\nabla_{6}\right\rangle & \left\langle\alpha \nabla_{2}+\nabla_{4}+\beta \nabla_{5}+\nabla_{6}\right\rangle_{\beta \neq 0} \\
\left\langle\nabla_{2}+\alpha \nabla_{5}+\nabla_{6}\right\rangle_{\alpha \neq 0} & \left\langle\nabla_{3}+\nabla_{6}\right\rangle & \left\langle\nabla_{5}\right\rangle \\
\left\langle\alpha \nabla_{5}+\nabla_{6}\right\rangle & \left\langle\nabla_{7}\right\rangle &
\end{array}
$$

\begin{tabular}{|c|c|c|c|c|c|c|c|}
\hline $\mathbf{N}_{26}^{4}(\alpha)$ & $:$ & $e_{1} e_{1}=e_{2}$ & $e_{1} e_{2}=e_{4}$ & $e_{1} e_{3}=-\alpha e_{4}$ & $e_{2} e_{1}=e_{3}$ & $e_{2} e_{2}=\alpha e_{4}$ & $e_{3} e_{1}=(1+2 \alpha) e_{4}$ \\
\hline $\mathbf{N}_{27}^{4}(\alpha, \beta)$ & $:$ & $e_{1} e_{1}=e_{2}$ & $e_{1} e_{2}=\alpha e_{4}$ & $e_{2} e_{1}=e_{3}$ & $e_{2} e_{2}=\beta e_{4}$ & $e_{2} e_{3}=e_{4}$ & $e_{3} e_{1}=e_{4}$ \\
\hline \multirow{2}{*}{$\mathbf{N}_{28}^{4}(\alpha, \beta, \gamma)$} & : & $e_{1} e_{1}=e_{2}$ & $e_{1} e_{2}=\alpha e_{4}$ & $e_{2} e_{1}=e_{3}$ & $e_{2} e_{2}=\beta e_{4}$ & & \\
\hline & & $e_{2} e_{3}=e_{4}$ & $e_{3} e_{1}=\gamma e_{4}$ & $e_{3} e_{3}=e_{4}$ & & & \\
\hline $\mathbf{N}_{29}^{4}(\alpha, \beta)$ & $:$ & $e_{1} e_{1}=e_{2}$ & $e_{1} e_{2}=\alpha e_{4}$ & $e_{2} e_{2}=\beta e_{4}$ & $e_{2} e_{1}=e_{3}$ & $e_{3} e_{1}=e_{4}$ & $e_{3} e_{3}=e_{4}$ \\
\hline $\mathbf{N}_{30}^{4}(\alpha)$ & $:$ & $e_{1} e_{1}=e_{2}$ & $e_{1} e_{2}=\alpha e_{4}$ & $e_{2} e_{1}=e_{3}$ & $e_{2} e_{2}=e_{4}$ & $e_{2} e_{3}=e_{4}$ & \\
\hline $\mathbf{N}_{31}^{4}(\alpha)$ & $:$ & $e_{1} e_{1}=e_{2}$ & $e_{1} e_{2}=\alpha e_{4}$ & $e_{2} e_{1}=e_{3}$ & $e_{2} e_{2}=e_{4}$ & $e_{3} e_{3}=e_{4}$ & \\
\hline $\mathbf{N}_{32}^{4}$ & $:$ & $e_{1} e_{1}=e_{2}$ & $e_{1} e_{2}=e_{4}$ & $e_{2} e_{1}=e_{3}$ & $e_{2} e_{3}=e_{4}$ & & \\
\hline $\mathbf{N}_{33}^{4}$ & $:$ & $e_{1} e_{1}=e_{2}$ & $e_{1} e_{2}=e_{4}$ & $e_{2} e_{1}=e_{3}$ & $e_{3} e_{3}=e_{4}$ & & \\
\hline $\mathbf{N}_{34}^{4}(\alpha, \beta)$ & $:$ & $e_{1} e_{1}=e_{2}$ & $e_{1} e_{3}=\beta e_{4}$ & $e_{2} e_{1}=e_{3}$ & $e_{2} e_{2}=\alpha e_{4}$ & $e_{3} e_{1}=(1-2 \beta)$ & \\
\hline $\mathbf{N}_{35}^{4}(\alpha)$ & $:$ & $e_{1} e_{1}=e_{2}$ & $e_{1} e_{3}=\alpha e_{4}$ & $e_{2} e_{1}=e_{3}$ & $e_{2} e_{2}=e_{4}$ & $e_{3} e_{1}=-2 \alpha e_{4}$ & $e_{3} e_{2}=e_{4}$ \\
\hline $\mathbf{N}_{36}^{4}(\alpha, \beta)_{\beta \neq 0}$ & $:$ & $e_{1} e_{1}=e_{2}$ & $e_{2} e_{1}=e_{3}$ & $e_{2} e_{2}=\alpha e_{4}$ & $e_{2} e_{3}=\beta e_{4}$ & $e_{3} e_{1}=e_{4}$ & $e_{3} e_{2}=e_{4}$ \\
\hline $\mathbf{N}_{37}^{4}(\alpha)_{\alpha \neq 0}$ & $:$ & $e_{1} e_{1}=e_{2}$ & $e_{2} e_{1}=e_{3}$ & $e_{2} e_{2}=e_{4}$ & $e_{2} e_{3}=\alpha e_{4}$ & $e_{3} e_{2}=e_{4}$ & \\
\hline $\mathbf{N}_{38}^{4}$ & $:$ & $e_{1} e_{1}=e_{2}$ & $e_{1} e_{3}=e_{4}$ & $e_{2} e_{1}=e_{3}$ & $e_{3} e_{1}=-2 e_{4}$ & $e_{3} e_{2}=e_{4}$ & \\
\hline $\mathbf{N}_{39}^{4}$ & $:$ & $e_{1} e_{1}=e_{2}$ & $e_{2} e_{1}=e_{3}$ & $e_{2} e_{3}=e_{4}$ & & & \\
\hline $\mathbf{N}_{40}^{4}(\alpha)$ & $:$ & $e_{1} e_{1}=e_{2}$ & $e_{2} e_{1}=e_{3}$ & $e_{2} e_{3}=\alpha e_{4}$ & $e_{3} e_{2}=e_{4}$ & & \\
\hline $\mathbf{N}_{41}^{4}$ & $:$ & $e_{1} e_{1}=e_{2}$ & $e_{2} e_{1}=e_{3}$ & $e_{3} e_{3}=e_{4}$ & & & \\
\hline
\end{tabular}

They correspond to the following new algebras: 


$$
\mathbf{N}_{29}^{4}(\alpha, \beta) \cong \mathbf{N}_{29}^{4}(-\alpha, \beta) \quad \mathbf{N}_{31}^{4}(\alpha) \cong \mathbf{N}_{31}^{4}(-\alpha)
$$

1.6. 1-dimensional central extensions of $\mathfrak{C} \mathfrak{D}_{04}^{3}$. Here we collect all information about $\mathfrak{C} \mathfrak{D}_{04}^{3}$ :

\begin{tabular}{|l|l|ll|}
\hline Algebra & Multiplication & Cohomology & \\
\hline & $e_{1} e_{1}=e_{2}$ & $\mathrm{H}_{\mathfrak{C D}}^{2}\left(\mathfrak{C D}_{04}^{3}\right)=\left\langle(\lambda-2) \Delta_{13}-(2 \lambda-1) \Delta_{31}, \Delta_{21}, \Delta_{22}\right\rangle$ \\
$\mathfrak{C D}_{04}^{3}$ & $e_{1} e_{2}=e_{3}$ & $\mathrm{H}^{2}\left(\mathfrak{C D}_{04}^{3}\right)=\mathrm{H}_{\mathfrak{C D}}^{2}\left(\mathfrak{C D}_{04}^{3}\right) \oplus\left\langle\left[\Delta_{13}\right]-2\left[\Delta_{31}\right],\left[\Delta_{23}\right],\left[\Delta_{32}\right],\left[\Delta_{33}\right]\right\rangle$ \\
& $e_{2} e_{1}=\lambda e_{3}$ & & \\
\hline
\end{tabular}

Let us use the following notations

$$
\begin{aligned}
& \nabla_{1}=(\lambda-2)\left[\Delta_{13}\right]-(2 \lambda-1)\left[\Delta_{31}\right] \quad \nabla_{2}=\left[\Delta_{21}\right] \quad \nabla_{3}=\left[\Delta_{22}\right] \\
& \nabla_{4}=\left[\Delta_{13}\right]-2\left[\Delta_{31}\right] \quad \nabla_{5}=\left[\Delta_{23}\right] \quad \nabla_{6}=\left[\Delta_{32}\right] \quad \nabla_{7}=\left[\Delta_{33}\right]
\end{aligned}
$$

Take $\theta=\sum_{i=1}^{3} \alpha_{i} \nabla_{i} \in \mathrm{H}_{\mathfrak{C D}}^{2}\left(\mathfrak{C D}_{04}^{3}\right)$. If

$$
\phi=\left(\begin{array}{ccc}
x & 0 & 0 \\
y & x^{2} & 0 \\
z & (\lambda+1) x y & x^{3}
\end{array}\right) \in \operatorname{Aut} \mathfrak{C D}_{04}^{3}
$$

then

$\phi^{T}\left(\begin{array}{ccc}0 & 0 & (\lambda-2) \alpha_{1}+\alpha_{4} \\ \alpha_{2} & \alpha_{3} & \alpha_{5} \\ -(2 \lambda-1) \alpha_{1}-2 \alpha_{4} & \alpha_{6} & \alpha_{7}\end{array}\right) \phi=\left(\begin{array}{ccc}\alpha^{*} & \alpha^{* *} & (\lambda-2) \alpha_{1}^{*}+\alpha_{4}^{*} \\ \alpha_{2}^{*}+\lambda \alpha^{* *} & \alpha_{3}^{*} & \alpha_{5}^{*} \\ -(2 \lambda-1) \alpha_{1}^{*}-2 \alpha_{4}^{*} & \alpha_{6}^{*} & \alpha_{7}^{*}\end{array}\right)$,

where

$$
\begin{aligned}
\alpha_{1}^{*}= & \frac{x^{3}}{3}\left(3 x \alpha_{1}-y\left(2 \alpha_{5}+\alpha_{6}\right)-3 z \alpha_{7}\right) \\
\alpha_{2}^{*}= & x\left(x^{2} \alpha_{2}+y(1+\lambda)\left(z \alpha_{7}(1-\lambda)+y\left(\alpha_{6}-\alpha_{5} \lambda\right)\right)+x\left(z\left(\alpha_{5}-\alpha_{6} \lambda\right)-y\left(\alpha_{3}(\lambda-1)+\right.\right.\right. \\
& \left.\left.\left.\alpha_{1}(\lambda-1)(\lambda+1)^{2}+\alpha_{4}\left(2+3 \lambda+\lambda^{2}\right)\right)\right)\right) \\
\alpha_{3}^{*}= & x^{2}\left(x^{2} \alpha_{3}+x y\left(\alpha_{5}+\alpha_{6}\right)(1+\lambda)+y^{2} \alpha_{7}(\lambda+1)^{2}\right) \\
\alpha_{4}^{*}= & \frac{x^{3}}{3}\left(3 x \alpha_{4}+y \alpha_{6}(\lambda-2)+3 z \alpha_{7}(\lambda-1)+y \alpha_{5}(2 \lambda-1)\right) \\
\alpha_{5}^{*}= & x^{4}\left(x \alpha_{5}+y \alpha_{7}(\lambda+1)\right) \\
\alpha_{6}^{*}= & x^{4}\left(x \alpha_{6}+y \alpha_{7}(\lambda+1)\right) \\
\alpha_{7}^{*}= & x^{6} \alpha_{7}
\end{aligned}
$$

Hence, $\phi\langle\theta\rangle=\left\langle\theta^{*}\right\rangle$, where $\theta^{*}=\sum_{i=1}^{7} \alpha_{i}^{*} \nabla_{i}$. We are only interested in elements with $\left(\alpha_{4}, \alpha_{5}, \alpha_{6}, \alpha_{7}\right) \neq(0,0,0,0)$.

(1) $\alpha_{7} \neq 0$ and $\lambda \neq-1$. We may assume $\alpha_{7}=1$. We put $z=\alpha_{1} x-\frac{1}{3}\left(2 \alpha_{5}+\alpha_{6}\right) y$ to make $\alpha_{1}^{*}=0$.

(a) $\alpha_{6} \neq \alpha_{5}$. Then choosing $y=-\frac{3\left((\lambda-1) \alpha_{1}+\alpha_{4}\right) x}{\alpha_{5}-\alpha_{6}}$ we have $\alpha_{4}^{*}=0$. Now, if $\alpha_{6} \neq$ $\frac{3(\lambda+1)\left((\lambda-1) \alpha_{1}+\alpha_{4}\right)}{\alpha_{5}-\alpha_{6}}$, then choosing $x=\alpha_{6}-\frac{3(\lambda+1)\left((\lambda-1) \alpha_{1}+\alpha_{4}\right)}{\alpha_{5}-\alpha_{6}}$ we have the family of 
representatives of distinct orbits $\left\langle\alpha \nabla_{2}+\beta \nabla_{3}+\gamma \nabla_{5}+\nabla_{6}+\nabla_{7}\right\rangle_{\lambda \neq-1, \gamma \neq 1}$. Otherwise, choosing $x=\alpha_{5}-\alpha_{6}$ we have the family of representatives of distinct orbits $\left\langle\alpha \nabla_{2}+\beta \nabla_{3}+\nabla_{5}+\nabla_{7}\right\rangle_{\lambda \neq-1}$.

(b) $\alpha_{6}=\alpha_{5}$ and $\alpha_{4} \neq(1-\lambda) \alpha_{1}$. Then choosing $y=-\frac{\alpha_{5} x}{\lambda+1}$ and $x=\sqrt{\alpha_{4}+(\lambda-1) \alpha_{1}}$, we have the family of representatives $\left\langle\alpha \nabla_{2}+\beta \nabla_{3}+\nabla_{4}+\nabla_{7}\right\rangle_{\lambda \neq-1}$, where $(\alpha, \beta)$ and $\left(\alpha^{\prime}, \beta^{\prime}\right)$ determine the same orbit if and only if $(\alpha, \beta)=\left( \pm \alpha^{\prime}, \beta^{\prime}\right)$.

(c) $\alpha_{6}=\alpha_{5}, \alpha_{4}=(1-\lambda) \alpha_{1}$ and $\alpha_{3} \neq \alpha_{5}^{2}$. Then choosing $y=-\frac{\alpha_{5} x}{\lambda+1}$ and $x=\sqrt{\alpha_{3}-\alpha_{5}^{2}}$, we have the family of representatives $\left\langle\alpha \nabla_{2}+\nabla_{3}+\nabla_{7}\right\rangle_{\lambda \neq-1}$, where $\alpha$ and $\alpha^{\prime}$ determine the same orbit if and only if $\alpha= \pm \alpha^{\prime}$.

(d) $\alpha_{6}=\alpha_{5}, \alpha_{4}=(1-\lambda) \alpha_{1}$ and $\alpha_{3}=\alpha_{5}^{2}$. Then have two representatives $\left\langle\nabla_{7}\right\rangle_{\lambda \neq-1}$ and $\left\langle\nabla_{2}+\nabla_{7}\right\rangle_{\lambda \neq-1}$ depending on whether $\alpha_{2}=(\lambda-1) \alpha_{1} \alpha_{5}$ or not.

(2) $\alpha_{7} \neq 0$ and $\lambda=-1$. We may assume $\alpha_{7}=1$.

(a) $\alpha_{6} \neq 0$ and $\alpha_{6} \neq \alpha_{5}$. Then choosing $x=\alpha_{6}, y=\frac{3\left(2 \alpha_{1}-\alpha_{4}\right) x}{\alpha_{5}-\alpha_{6}}$ and $z=$ $\frac{\left(\alpha_{4}\left(2 \alpha_{5}+\alpha_{6}\right)-3 \alpha_{1}\left(\alpha_{5}+\alpha_{6}\right)\right) x}{\alpha_{5}-\alpha_{6}}$, we have the family of representatives of distinct orbits $\left\langle\alpha \nabla_{2}+\right.$ $\left.\beta \nabla_{3}+\gamma \nabla_{5}+\nabla_{6}+\nabla_{7}\right\rangle_{\lambda=-1, \gamma \neq 1}$.

(b) $\alpha_{6} \neq 0, \alpha_{6}=\alpha_{5}$ and $\alpha_{3} \neq \alpha_{5}^{2}$. Then choosing $x=\alpha_{5}, y=\frac{\left(\alpha_{2}+2 \alpha_{1} \alpha_{5}\right) x}{2\left(\alpha_{5}^{2}-\alpha_{3}\right)}$ and $z=$ $\frac{\left(\alpha_{2} \alpha_{5}+2 \alpha_{1} \alpha_{3}\right) x}{2\left(\alpha_{3}-\alpha_{5}^{2}\right)}$, we have the family of representatives of distinct orbits $\left\langle\alpha \nabla_{3}+\beta \nabla_{4}+\right.$ $\left.\nabla_{5}+\nabla_{6}+\nabla_{7}\right\rangle_{\lambda=-1, \alpha \neq 1}$.

(c) $\alpha_{6} \neq 0, \alpha_{6}=\alpha_{5}$ and $\alpha_{3}=\alpha_{5}^{2}$. Then choosing $x=\alpha_{6}, y=\alpha_{1}$ and $z=0$, we have the family of representatives of distinct orbits $\left\langle\alpha \nabla_{2}+\nabla_{3}+\beta \nabla_{4}+\nabla_{5}+\nabla_{6}+\nabla_{7}\right\rangle_{\lambda=-1}$.

(d) $\alpha_{6}=0$ and $\alpha_{5} \neq 0$. Then choosing $x=\alpha_{5}, y=3\left(2 \alpha_{1}-\alpha_{4}\right)$ and $z=\left(2 \alpha_{4}-3 \alpha_{1}\right) \alpha_{5}$, we have the family of representatives of distinct orbits $\left\langle\alpha \nabla_{2}+\beta \nabla_{3}+\nabla_{5}+\nabla_{7}\right\rangle_{\lambda=-1}$.

(e) $\alpha_{6}=0, \alpha_{5}=0, \alpha_{4} \neq 2 \alpha_{1}$ and $\alpha_{3} \neq 0$. Then choosing $x=\sqrt{\alpha_{4}-2 \alpha_{1}}, y=-\frac{\alpha_{2} x}{2 \alpha_{3}}$ and $z=\alpha_{1} x$, we have the family of representatives of distinct orbits $\left\langle\alpha \nabla_{3}+\nabla_{4}+\right.$ $\left.\nabla_{7}\right\rangle_{\lambda=-1, \alpha \neq 0}$.

(f) $\alpha_{6}=0, \alpha_{5}=0, \alpha_{4}=2 \alpha_{1}$ and $\alpha_{3} \neq 0$. Then choosing $x=\sqrt{\alpha_{3}}, y=-\frac{\alpha_{2} x}{2 \alpha_{3}}$ and $z=\alpha_{1} x$, we have the representative $\left\langle\nabla_{3}+\nabla_{7}\right\rangle_{\lambda=-1}$.

(g) $\alpha_{6}=0, \alpha_{5}=0, \alpha_{4}=2 \alpha_{1}$ and $\alpha_{3}=0$. Then choosing $z=\alpha_{1} x$, we have two representatives $\left\langle\nabla_{7}\right\rangle_{\lambda=-1}$ and $\left\langle\nabla_{2}+\nabla_{7}\right\rangle_{\lambda=-1}$, depending on whether $\alpha_{2}=0$ or not.

(3) $\alpha_{7}=0$ and $\alpha_{6} \neq 0$. We may assume $\alpha_{6}=1$.

(a) $\alpha_{5} \notin\left\{-\frac{1}{2}, \lambda\right\}$. Then the system $\alpha_{1}^{*}=\alpha_{2}^{*}=0$ has a unique solution in $y$ and $z$. Now, if $\alpha_{4} \neq-\frac{\left((2 \lambda-1) \alpha_{5}+\lambda-2\right) \alpha_{1}}{2 \alpha_{5}+1}$, then choosing the suitable value of $x$ we have the family of representatives distinct orbits $\left\langle\alpha \nabla_{3}+\nabla_{4}+\beta \nabla_{5}+\nabla_{6}\right\rangle_{\beta \notin\left\{-\frac{1}{2}, \lambda\right\}}$. Otherwise, we have two families of representatives distinct orbits $\left\langle\alpha \nabla_{5}+\nabla_{6}\right\rangle_{\alpha \notin\left\{-\frac{1}{2}, \lambda\right\}}$ and $\left\langle\nabla_{3}+\alpha \nabla_{5}+\right.$ $\left.\nabla_{6}\right\rangle_{\alpha \notin\left\{-\frac{1}{2}, \lambda\right\}}$ depending on whether $\alpha_{3}=-\frac{3(\lambda+1)\left(\alpha_{5}+1\right) \alpha_{1}}{2 \alpha_{5}+1}$ or not.

(b) $\alpha_{5}=\lambda \neq-\frac{1}{2}$ and $\alpha_{4} \neq \frac{2\left(1-\lambda^{2}\right) \alpha_{1}}{2 \lambda+1}$. Then choosing $x=\alpha_{4}+\frac{2\left(\lambda^{2}-1\right) \alpha_{1}}{2 \lambda+1}$ and $y=\frac{3 \alpha_{1} x}{2 \lambda+1}$ we have the family of representatives of distinct orbits $\left\langle\alpha \nabla_{2}+\beta \nabla_{3}+\nabla_{4}+\lambda \nabla_{5}+\nabla_{6}\right\rangle_{\lambda \neq-\frac{1}{2}}$. 
(c) $\alpha_{5}=\lambda \neq-\frac{1}{2}$ and $\alpha_{4}=\frac{2\left(1-\lambda^{2}\right) \alpha_{1}}{2 \lambda+1}$. We put $y=\frac{3 \alpha_{1} x}{2 \lambda+1}$ to make $\alpha_{1}^{*}=0$. Now, if $\alpha_{3} \neq-\frac{3(\lambda+1)^{2} \alpha_{1}}{2 \lambda+1}$, then choosing $x=\alpha_{3}+\frac{3(\lambda+1)^{2} \alpha_{1}}{2 \lambda+1}$, we have the family of representatives of distinct orbits $\left\langle\alpha \nabla_{2}+\nabla_{3}+\lambda \nabla_{5}+\nabla_{6}\right\rangle_{\lambda \neq-\frac{1}{2}}$. Otherwise, we have two representatives $\left\langle\lambda \nabla_{5}+\nabla_{6}\right\rangle_{\lambda \neq-\frac{1}{2}}$ and $\left\langle\nabla_{2}+\lambda \nabla_{5}+\nabla_{6}\right\rangle_{\lambda \neq-\frac{1}{2}}$, depending on whether $\alpha_{2}=-\frac{9(\lambda-1)(\lambda+1)^{2} \alpha_{1}^{2}}{(2 \lambda+1)^{2}}$ or not.

(d) $\alpha_{5}=-\frac{1}{2} \neq \lambda$. Then put $y=2 \alpha_{4} x$ and $z=\frac{2\left(\alpha_{2}-2 \alpha_{4}(\lambda-1)\left(\alpha_{3}+\alpha_{1}(\lambda+1)^{2}\right) x\right.}{2 \lambda+1}$ to make $\alpha_{2}^{*}=$ $\alpha_{4}^{*}=0$. Now, if $\alpha_{3} \neq-(\lambda+1) \alpha_{4}$, then choosing $x=\alpha_{3}+(\lambda+1) \alpha_{4}$, we have the family of representatives of distinct orbits $\left\langle\alpha \nabla_{1}+\nabla_{3}-\frac{1}{2} \nabla_{5}+\nabla_{6}\right\rangle_{\lambda \neq-\frac{1}{2}}$. Otherwise, we have two representatives $\left\langle-\frac{1}{2} \nabla_{5}+\nabla_{6}\right\rangle_{\lambda \neq-\frac{1}{2}}$ and $\left\langle\nabla_{1}-\frac{1}{2} \nabla_{5}+\nabla_{6}\right\rangle_{\lambda \neq-\frac{1}{2}}$, depending on whether $\alpha_{1}=0$ or not.

(e) $\alpha_{5}=-\frac{1}{2}=\lambda$ and $\alpha_{4} \neq-2 \alpha_{3}$. Then choosing $x=\alpha_{3}+\frac{\alpha_{4}}{2}$ and $y=2 \alpha_{4} x$ we have the family of representatives of distinct orbits $\left\langle\alpha \nabla_{1}+\beta \nabla_{2}+\nabla_{3}-\frac{1}{2} \nabla_{5}+\nabla_{6}\right\rangle_{\lambda=-\frac{1}{2}}$.

(f) $\alpha_{5}=-\frac{1}{2}=\lambda$ and $\alpha_{4}=-2 \alpha_{3}$. Then we put $y=-4 \alpha_{3} x$ to make $\alpha_{4}^{*}=0$. Now, if $\alpha_{2} \neq \frac{3 \alpha_{3}}{2}\left(\alpha_{1}+4 \alpha_{3}\right)$, then choosing $x=\sqrt{\alpha_{2}-\frac{3 \alpha_{3}}{2}\left(\alpha_{1}+4 \alpha_{3}\right)}$, we have the family of representatives of distinct orbits $\left\langle\alpha \nabla_{1}+\nabla_{2}-\frac{1}{2} \nabla_{5}+\nabla_{6}\right\rangle_{\lambda=-\frac{1}{2}}$. Otherwise, we have two representatives $\left\langle\nabla_{1}-\frac{1}{2} \nabla_{5}+\nabla_{6}\right\rangle_{\lambda=-\frac{1}{2}}$ and $\left\langle-\frac{1}{2} \nabla_{5}+\nabla_{6}\right\rangle_{\lambda=-\frac{1}{2}}$, depending on whether $\alpha_{1}=0$ or not.

(4) $\alpha_{7}=\alpha_{6}=0$ and $\alpha_{5} \neq 0$. We may assume $\alpha_{5}=1$. Then the system $\alpha_{1}^{*}=\alpha_{2}^{*}=0$ has a unique solution in $y$ and $z$. Now, if $2 \alpha_{4}+(2 \lambda-1) \alpha_{1} \neq 0$, then choosing $x=\alpha_{4}+\left(\lambda-\frac{1}{2}\right) \alpha_{1}$ we have the family of representatives of distinct orbits $\left\langle\alpha \nabla_{3}+\nabla_{4}+\nabla_{5}\right\rangle$. Otherwise, we have two representatives $\left\langle\nabla_{5}\right\rangle$ and $\left\langle\nabla_{3}+\nabla_{5}\right\rangle$ depending on whether $2 \alpha_{3}+3(\lambda+1) \alpha_{1}=0$ or not. (5) $\alpha_{7}=\alpha_{6}=\alpha_{5}=0$ and $\alpha_{4} \neq 0$. We may assume $\alpha_{4}=1$.

(a) $(\lambda-1)\left((\lambda+1)^{2} \alpha_{1}+\alpha_{3}\right)+(\lambda+1)(\lambda+2) \neq 0$. Then choosing $y=$ $\frac{\alpha_{2} x}{(\lambda-1)\left((\lambda+1)^{2} \alpha_{1}+\alpha_{3}\right)+(\lambda+1)(\lambda+2)}$ we have the family of representatives of distinct orbits $\left\langle\alpha \nabla_{1}+\beta \nabla_{3}+\nabla_{4}\right\rangle$, where $(\lambda-1)\left((\lambda+1)^{2} \alpha+\beta\right)+(\lambda+1)(\lambda+2) \neq 0$.

(b) $(\lambda-1)\left((\lambda+1)^{2} \alpha_{1}+\alpha_{3}\right)+(\lambda+1)(\lambda+2)=0$. Then $\lambda \neq 1$ and we have two families of representatives of distinct orbits $\left\langle\alpha \nabla_{1}+\frac{(\lambda+1)\left(\left(\lambda^{2}-1\right) \alpha+\lambda+2\right)}{1-\lambda} \nabla_{3}+\nabla_{4}\right\rangle_{\lambda \neq 1}$ and $\left\langle\alpha \nabla_{1}+\nabla_{2}+\frac{(\lambda+1)\left(\left(\lambda^{2}-1\right) \alpha+\lambda+2\right)}{1-\lambda} \nabla_{3}+\nabla_{4}\right\rangle_{\lambda \neq 1}$, depending on whether $\alpha_{2}=0$ or not. The first family will be joined with the family from the previous item.

Summarizing, we have the following representatives of distinct orbits:

$$
\begin{array}{ll}
\left\langle\alpha \nabla_{1}+\nabla_{2}+\frac{(\lambda+1)\left(\left(\lambda^{2}-1\right) \alpha+\lambda+2\right)}{1-\lambda} \nabla_{3}+\nabla_{4}\right\rangle_{\lambda \neq 1} & \left\langle\alpha \nabla_{1}+\beta \nabla_{2}+\nabla_{3}-\frac{1}{2} \nabla_{5}+\nabla_{6}\right\rangle_{\lambda=-\frac{1}{2}} \\
\left\langle\alpha \nabla_{1}+\nabla_{2}-\frac{1}{2} \nabla_{5}+\nabla_{6}\right\rangle_{\lambda=-\frac{1}{2}} & \left\langle\alpha \nabla_{1}+\beta \nabla_{3}+\nabla_{4}\right\rangle \\
\left\langle\alpha \nabla_{1}+\nabla_{3}-\frac{1}{2} \nabla_{5}+\nabla_{6}\right\rangle_{\lambda \neq-\frac{1}{2}} & \left\langle\nabla_{1}-\frac{1}{2} \nabla_{5}+\nabla_{6}\right\rangle_{\lambda=-\frac{1}{2}} \\
\left\langle\alpha \nabla_{2}+\beta \nabla_{3}+\nabla_{4}+\lambda \nabla_{5}+\nabla_{6}\right\rangle_{\lambda \neq-\frac{1}{2}} & \left\langle\alpha \nabla_{2}+\nabla_{3}+\beta \nabla_{4}+\nabla_{5}+\nabla_{6}+\nabla_{7}\right\rangle_{\lambda=-1}
\end{array}
$$



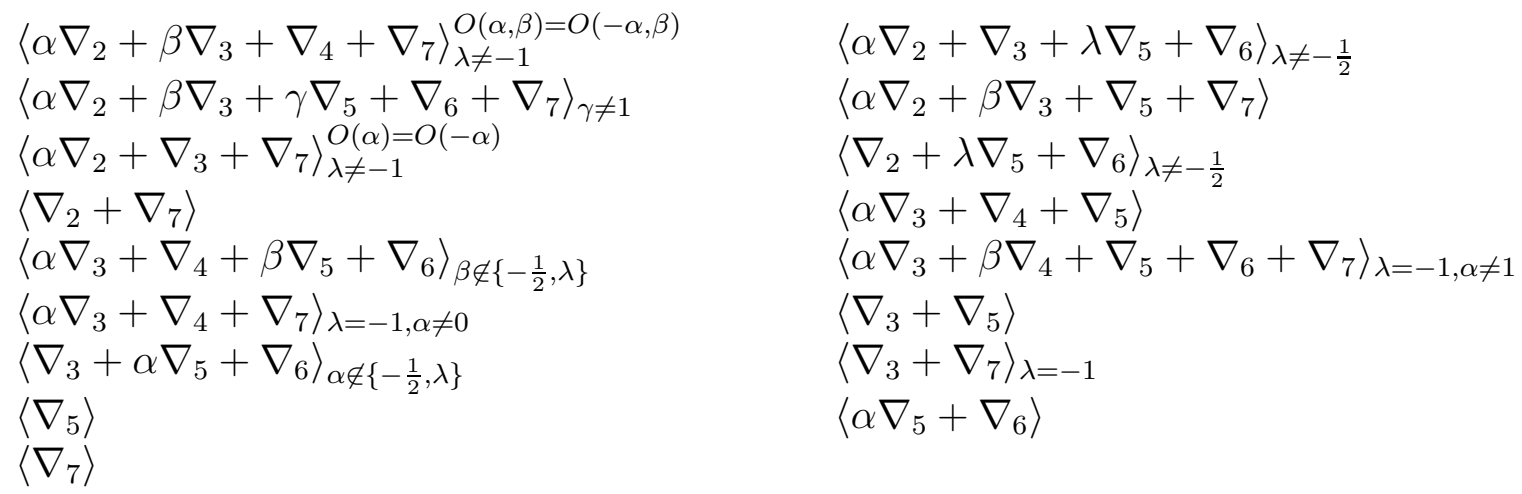

The corresponding algebras are:

\begin{tabular}{|c|c|c|c|c|c|}
\hline $\mathbf{N}_{42}^{4}(\lambda, \alpha)_{\lambda \neq 1}$ & $:$ & $\begin{array}{l}e_{1} e_{1}=e_{2} \\
e_{2} e_{1}=\lambda e_{3}+e_{4}\end{array}$ & $\begin{array}{l}e_{1} e_{2}=e_{3} \\
e_{2} e_{2}=\underline{(\lambda+1)(}\end{array}$ & $\frac{\left.\left.\lambda^{2}-1\right) \alpha+\lambda+2\right)}{1-\lambda} e_{4}$ & $\begin{array}{l}e_{1} e_{3}=(\alpha(\lambda-2)+1) e_{4} \\
e_{3} e_{1}=(\alpha(1-2 \lambda)-2) e_{4}\end{array}$ \\
\hline $\mathbf{N}_{43}^{4}(\alpha, \beta)$ & $:$ & $\begin{array}{l}e_{1} e_{1}=e_{2} \\
e_{2} e_{2}=e_{4}\end{array}$ & $\begin{array}{l}e_{1} e_{2}=e_{3} \\
e_{2} e_{3}=-\frac{1}{2} e_{4}\end{array}$ & $\begin{array}{l}e_{1} e_{3}=-\frac{5 \alpha}{2} e_{4} \\
e_{3} e_{1}=2 \alpha e_{4}\end{array}$ & $\begin{array}{l}e_{2} e_{1}=-\frac{1}{2} e_{3}+\beta e_{4} \\
e_{3} e_{2}=e_{4}\end{array}$ \\
\hline $\mathbf{N}_{44}^{4}(\alpha)$ & $:$ & $\begin{array}{l}e_{1} e_{1}=e_{2} \\
e_{2} e_{3}=-\frac{1}{2} e_{4}\end{array}$ & $\begin{array}{l}e_{1} e_{2}=e_{3} \\
e_{3} e_{1}=2 \alpha e_{4}\end{array}$ & $\begin{array}{l}e_{1} e_{3}=-\frac{5 \alpha}{2} e_{4} \\
e_{3} e_{2}=e_{4}\end{array}$ & $e_{2} e_{1}=-\frac{1}{2} e_{3}+e_{4}$ \\
\hline $\mathbf{N}_{45}^{4}(\lambda, \alpha, \beta)$ & $:$ & $\begin{array}{l}e_{1} e_{1}=e_{2} \\
e_{2} e_{1}=\lambda e_{3}\end{array}$ & $\begin{array}{l}e_{1} e_{2}=e_{3} \\
e_{2} e_{2}=\beta e_{4}\end{array}$ & $\begin{array}{l}e_{1} e_{3}=(\alpha(\lambda-2)+ \\
e_{3} e_{1}=(\alpha(1-2 \lambda)\end{array}$ & $\begin{array}{l}e_{4} \\
2) e_{4} \\
\end{array}$ \\
\hline $\mathbf{N}_{46}^{4}(\lambda, \alpha)_{\lambda \neq-\frac{1}{2}}$ & $\overline{:}$ & $\begin{array}{l}e_{1} e_{1}=e_{2} \\
e_{2} e_{2}=e_{4}\end{array}$ & $\begin{array}{l}e_{1} e_{2}=e_{3} \\
e_{2} e_{3}=-\frac{1}{2} e_{4}\end{array}$ & $\begin{array}{l}e_{1} e_{3}=\alpha(\lambda-2) e_{4} \\
e_{3} e_{1}=\alpha(1-2 \lambda) e_{4}\end{array}$ & $\begin{array}{l}e_{2} e_{1}=\lambda e_{3} \\
e_{3} e_{2}=e_{4}\end{array}$ \\
\hline $\mathbf{N}_{47}^{4}$ & 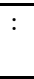 & $\begin{array}{l}e_{1} e_{1}=e_{2} \\
e_{2} e_{3}=-\frac{1}{2} e_{4}\end{array}$ & $\begin{array}{l}e_{1} e_{2}=e_{3} \\
e_{3} e_{1}=2 e_{4}\end{array}$ & $\begin{array}{l}e_{1} e_{3}=-\frac{5}{2} e_{4} \\
e_{3} e_{2}=e_{4}\end{array}$ & $e_{2} e_{1}=-\frac{1}{2} e_{3}$ \\
\hline $\mathbf{N}_{48}^{4}(\lambda, \alpha, \beta)_{\lambda \neq-\frac{1}{2}}$ & $:$ & $\begin{array}{l}e_{1} e_{1}=e_{2} \\
e_{2} e_{2}=\beta e_{4}\end{array}$ & $\begin{array}{l}e_{1} e_{2}=e_{3} \\
e_{2} e_{3}=\lambda e_{4}\end{array}$ & $\begin{array}{l}e_{1} e_{3}=e_{4} \\
e_{3} e_{1}=-2 e_{4}\end{array}$ & $\begin{array}{l}e_{2} e_{1}=\lambda e_{3}+\alpha e_{4} \\
e_{3} e_{2}=e_{4}\end{array}$ \\
\hline $\mathbf{N}_{49}^{4}(\alpha, \beta)$ & $:$ & $\begin{array}{l}e_{1} e_{1}=e_{2} \\
e_{2} e_{1}=-e_{3}+\alpha e_{4} \\
e_{3} e_{1}=-2 \beta e_{4}\end{array}$ & $\begin{array}{l}e_{1} e_{2}=e_{3} \\
e_{2} e_{2}=e_{4} \\
e_{3} e_{2}=e_{4}\end{array}$ & $\begin{array}{l}e_{1} e_{3}=\beta e_{4} \\
e_{2} e_{3}=e_{4} \\
e_{3} e_{3}=e_{4}\end{array}$ & \\
\hline $\mathbf{N}_{50}^{4}(\lambda, \alpha, \beta)_{\lambda \neq-1}$ & $:$ & $\begin{array}{l}e_{1} e_{1}=e_{2} \\
e_{2} e_{2}=\beta e_{4}\end{array}$ & $\begin{array}{l}e_{1} e_{2}=e_{3} \\
e_{3} e_{1}=-2 e_{4}\end{array}$ & $\begin{array}{l}e_{1} e_{3}=e_{4} \\
e_{3} e_{3}=e_{4}\end{array}$ & $e_{2} e_{1}=\lambda e_{3}+\alpha e_{4}$ \\
\hline $\mathbf{N}_{51}^{4}(\lambda, \alpha)_{\lambda \neq-\frac{1}{2}}$ & $\overline{:}$ & $\begin{array}{l}e_{1} e_{1}=e_{2} \\
e_{2} e_{2}=e_{4}\end{array}$ & $\begin{array}{l}e_{1} e_{2}=e_{3} \\
e_{2} e_{3}=\lambda e_{4}\end{array}$ & $\begin{array}{l}e_{2} e_{1}=\lambda e_{3}+\alpha e_{4} \\
e_{3} e_{2}=e_{4}\end{array}$ & \\
\hline $\mathbf{N}_{52}^{4}(\lambda, \alpha, \beta, \gamma)_{\gamma \neq 1}$ & $:$ & $\begin{array}{l}e_{1} e_{1}=e_{2} \\
e_{2} e_{3}=\gamma e_{4}\end{array}$ & $\begin{array}{l}e_{1} e_{2}=e_{3} \\
e_{3} e_{2}=e_{4}\end{array}$ & $\begin{array}{l}e_{2} e_{1}=\lambda e_{3}+\alpha e_{4} \\
e_{3} e_{3}=e_{4}\end{array}$ & $e_{2} e_{2}=\beta e_{4}$ \\
\hline $\mathbf{N}_{53}^{4}(\lambda, \alpha, \beta)$ & $:$ & $\begin{array}{l}e_{1} e_{1}=e_{2} \\
e_{2} e_{2}=\beta e_{4}\end{array}$ & $\begin{array}{l}e_{1} e_{2}=e_{3} \\
e_{2} e_{3}=e_{4}\end{array}$ & $\begin{array}{l}e_{2} e_{1}=\lambda e_{3}+\alpha e_{4} \\
e_{3} e_{3}=e_{4}\end{array}$ & \\
\hline $\mathbf{N}_{54}^{4}(\lambda, \alpha)_{\lambda \neq-1}$ & $\overline{:}$ & $\begin{array}{l}e_{1} e_{1}=e_{2} \\
e_{2} e_{2}=e_{4}\end{array}$ & $\begin{array}{l}e_{1} e_{2}=e_{3} \\
e_{3} e_{3}=e_{4}\end{array}$ & $e_{2} e_{1}=\lambda e_{3}+\alpha e_{4}$ & \\
\hline $\mathbf{N}_{55}^{4}(\lambda)_{\lambda \neq-\frac{1}{2}}$ & $:$ & $\begin{array}{l}e_{1} e_{1}=e_{2} \\
e_{2} e_{3}=\lambda e_{4}\end{array}$ & $\begin{array}{l}e_{1} e_{2}=e_{3} \\
e_{3} e_{2}=e_{4}\end{array}$ & $e_{2} e_{1}=\lambda e_{3}+e_{4}$ & \\
\hline $\mathbf{N}_{56}^{4}(\lambda)$ & $:$ & $e_{1} e_{1}=e_{2}$ & $e_{1} e_{2}=e_{3}$ & $e_{2} e_{1}=\lambda e_{3}+e_{4}$ & $e_{3} e_{3}=e_{4}$ \\
\hline $\mathbf{N}_{57}^{4}(\lambda, \alpha)$ & $\overline{:}$ & $\begin{array}{l}e_{1} e_{1}=e_{2} \\
e_{2} e_{2}=\alpha e_{4}\end{array}$ & $\begin{array}{l}e_{1} e_{2}=e_{3} \\
e_{2} e_{3}=e_{4}\end{array}$ & $\begin{array}{l}e_{1} e_{3}=e_{4} \\
e_{3} e_{1}=-2 e_{4}\end{array}$ & $e_{2} e_{1}=\lambda e_{3}$ \\
\hline $\mathbf{N}_{58}^{4}(\lambda, \alpha, \beta)_{\beta \notin\left\{-\frac{1}{2}, \lambda\right\}}$ & $:$ & $\begin{array}{l}e_{1} e_{1}=e_{2} \\
e_{2} e_{2}=\alpha e_{4}\end{array}$ & $\begin{array}{l}e_{1} e_{2}=e_{3} \\
e_{2} e_{3}=\beta e_{4}\end{array}$ & $\begin{array}{l}e_{1} e_{3}=e_{4} \\
e_{3} e_{1}=-2 e_{4}\end{array}$ & $\begin{array}{l}e_{2} e_{1}=\lambda e_{3} \\
e_{3} e_{2}=e_{4}\end{array}$ \\
\hline $\mathbf{N}_{59}^{4}(\alpha, \beta)_{\alpha \neq 1}$ & $:$ & $\begin{array}{l}e_{1} e_{1}=e_{2} \\
e_{2} e_{1}=-e_{3} \\
e_{3} e_{1}=-2 \beta e_{4}\end{array}$ & $\begin{array}{l}e_{1} e_{2}=e_{3} \\
e_{2} e_{2}=\alpha e_{4} \\
e_{3} e_{2}=e_{4}\end{array}$ & $\begin{array}{l}e_{1} e_{3}=\beta e_{4} \\
e_{2} e_{3}=e_{4} \\
e_{3} e_{3}=e_{4}\end{array}$ & \\
\hline
\end{tabular}




\begin{tabular}{|c|c|c|c|c|c|}
\hline $\mathbf{N}_{60}^{4}(\alpha)_{\alpha \neq 0}$ & $:$ & $\begin{array}{l}e_{1} e_{1}=e_{2} \\
e_{2} e_{2}=\alpha e_{4}\end{array}$ & $\begin{array}{l}e_{1} e_{2}=e_{3} \\
e_{3} e_{1}=-2 e_{4}\end{array}$ & $\begin{array}{l}e_{1} e_{3}=e_{4} \\
e_{3} e_{3}=e_{4}\end{array}$ & $e_{2} e_{1}=-e_{3}$ \\
\hline $\mathbf{N}_{61}^{4}(\lambda)$ & $:$ & $\begin{array}{l}e_{1} e_{1}=e_{2} \\
e_{2} e_{2}=e_{4}\end{array}$ & $\begin{array}{l}e_{1} e_{2}=e_{3} \\
e_{2} e_{3}=e_{4}\end{array}$ & $e_{2} e_{1}=\lambda e_{3}$ & \\
\hline $\mathbf{N}_{62}^{4}(\lambda, \alpha)_{\alpha \notin\left\{-\frac{1}{2}, \lambda\right\}}$ & $:$ & $\begin{array}{l}e_{1} e_{1}=e_{2} \\
e_{2} e_{2}=e_{4}\end{array}$ & $\begin{array}{l}e_{1} e_{2}=e_{3} \\
e_{2} e_{3}=\alpha e_{4}\end{array}$ & $\begin{array}{l}e_{2} e_{1}=\lambda e_{3} \\
e_{3} e_{2}=e_{4}\end{array}$ & \\
\hline $\mathbf{N}_{63}^{4}$ & $:$ & $\begin{array}{l}e_{1} e_{1}=e_{2} \\
e_{2} e_{2}=e_{4}\end{array}$ & $\begin{array}{l}e_{1} e_{2}=e_{3} \\
e_{3} e_{3}=e_{4}\end{array}$ & $e_{2} e_{1}=-e_{3}$ & \\
\hline $\mathbf{N}_{64}^{4}(\lambda)$ & $:$ & $e_{1} e_{1}=e_{2}$ & $e_{1} e_{2}=e_{3}$ & $e_{2} e_{1}=\lambda e_{3}$ & $e_{2} e_{3}=e_{4}$ \\
\hline $\mathbf{N}_{65}^{4}(\lambda, \alpha)$ & $:$ & $\begin{array}{l}e_{1} e_{1}=e_{2} \\
e_{2} e_{3}=\alpha e_{4}\end{array}$ & $\begin{array}{l}e_{1} e_{2}=e_{3} \\
e_{3} e_{2}=e_{4}\end{array}$ & $e_{2} e_{1}=\lambda e_{3}$ & \\
\hline $\mathbf{N}_{66}^{4}(\lambda)$ & $:$ & $e_{1} e_{1}=e_{2}$ & $e_{1} e_{2}=e_{3}$ & $e_{2} e_{1}=\lambda e_{3}$ & $e_{3} e_{3}=e_{4}$ \\
\hline
\end{tabular}

$$
\mathbf{N}_{50}^{4}(\lambda, \alpha, \beta) \cong \mathbf{N}_{50}^{4}(\lambda,-\alpha, \beta) \quad \mathbf{N}_{54}^{4}(\lambda, \alpha) \cong \mathbf{N}_{54}^{4}(\lambda,-\alpha)
$$

\section{THE CLASSIFICATION THEOREM}

Theorem 2. Let $\mathbf{N}$ be a complex 4-dimensional nilpotent algebra. Then $\mathbf{N}$ is isomorphic to an algebra from the following list:

\begin{tabular}{|c|c|c|c|c|c|}
\hline $\mathfrak{C} \mathfrak{D}_{01}^{4 *}$ & $:$ & $e_{1} e_{1}=e_{2}$ & & & \\
\hline $\mathfrak{C} \mathfrak{D}_{02}^{4 *}$ & $:$ & $e_{1} e_{1}=e_{3}$ & $e_{2} e_{2}=e_{3}$ & & \\
\hline $\mathfrak{C} \mathfrak{D}_{03}^{4 *}$ & $:$ & $e_{1} e_{2}=e_{3}$ & $e_{2} e_{1}=-e_{3}$ & & \\
\hline $\mathfrak{C} \mathfrak{D}_{04}^{4 *}(\lambda)$ & $:$ & $e_{1} e_{1}=\lambda e_{3}$ & $e_{2} e_{1}=e_{3}$ & $e_{2} e_{2}=e_{3}$ & \\
\hline $\mathfrak{C} \mathfrak{D}_{05}^{4 *}$ & $:$ & $e_{1} e_{1}=e_{3}$ & $e_{2} e_{2}=e_{4}$ & & \\
\hline $\mathfrak{C D}_{06}^{4 *}$ & $:$ & $e_{1} e_{2}=e_{4}$ & $e_{3} e_{1}=e_{4}$ & & \\
\hline $\mathfrak{C D}_{07}^{4 *}$ & $:$ & $e_{1} e_{2}=e_{3}$ & $e_{2} e_{1}=e_{4}$ & $e_{2} e_{2}=-e_{3}$ & \\
\hline $\mathfrak{C}^{2} \mathfrak{D}_{08}^{4 *}(\alpha)$ & $:$ & $e_{1} e_{1}=e_{3}$ & $e_{1} e_{2}=e_{4}$ & $e_{2} e_{1}=-\alpha e_{3}$ & $e_{2} e_{2}=-e_{4}$ \\
\hline $\mathfrak{C} \mathfrak{D}_{09}^{4 *}(\alpha)$ & : & $\begin{array}{l}e_{1} e_{1}=e_{4} \\
e_{2} e_{2}=e_{4}\end{array}$ & $\begin{array}{l}e_{1} e_{2}=\alpha e_{4} \\
e_{3} e_{3}=e_{4}\end{array}$ & $e_{2} e_{1}=-\alpha e_{4}$ & \\
\hline $\mathfrak{C D}_{10}^{4 *}$ & $:$ & $\begin{array}{l}e_{1} e_{2}=e_{4} \\
e_{2} e_{2}=e_{4}\end{array}$ & $\begin{array}{l}e_{1} e_{3}=e_{4} \\
e_{3} e_{1}=e_{4}\end{array}$ & $e_{2} e_{1}=-e_{4}$ & \\
\hline $\mathfrak{C D}_{11}^{4 *}$ & $:$ & $e_{1} e_{1}=e_{4}$ & $e_{1} e_{2}=e_{4}$ & $e_{2} e_{1}=-e_{4}$ & $e_{3} e_{3}=e_{4}$ \\
\hline $\mathfrak{C D}_{12}^{4 *}$ & $:$ & $e_{1} e_{2}=e_{3}$ & $e_{2} e_{1}=e_{4}$ & & \\
\hline $\mathfrak{C D}_{13}^{4 *}$ & $:$ & $e_{1} e_{1}=e_{4}$ & $e_{1} e_{2}=e_{3}$ & $e_{2} e_{1}=-e_{3}$ & $e_{2} e_{2}=2 e_{3}+e_{4}$ \\
\hline $\mathfrak{C D}_{14}^{4 *}(\alpha)$ & $:$ & $e_{1} e_{2}=e_{4}$ & $e_{2} e_{1}=\alpha e_{4}$ & $e_{2} e_{2}=e_{3}$ & \\
\hline $\mathfrak{C D}_{15}^{4 *}$ & $:$ & $e_{1} e_{2}=e_{4}$ & $e_{2} e_{1}=-e_{4}$ & $e_{3} e_{3}=e_{4}$ & \\
\hline $\mathbf{D}_{00}^{4}$ & $:$ & $\begin{array}{l}e_{1} e_{1}=e_{4} \\
e_{2} e_{2}=e_{3}\end{array}$ & $\begin{array}{l}e_{1} e_{2}=e_{4} \\
e_{2} e_{3}=e_{4}\end{array}$ & $e_{2} e_{1}=e_{3}$ & \\
\hline$\overline{\mathbf{D}_{01}^{4}(\lambda, \alpha, \beta)}$ & : & $\begin{array}{l}e_{1} e_{1}=\lambda e_{3}+e_{4} \\
e_{2} e_{2}=e_{3}\end{array}$ & $\begin{array}{l}e_{1} e_{3}=\alpha e_{4} \\
e_{2} e_{3}=\beta e_{4}\end{array}$ & $\begin{array}{l}e_{2} e_{1}=e_{3} \\
e_{3} e_{1}=e_{4}\end{array}$ & \\
\hline $\mathbf{D}_{02}^{4}(\lambda, \alpha, \beta)$ & : & $\begin{array}{l}e_{1} e_{1}=\lambda e_{3} \\
e_{2} e_{2}=e_{3}\end{array}$ & $\begin{array}{l}e_{1} e_{3}=\alpha e_{4} \\
e_{2} e_{3}=\beta e_{4}\end{array}$ & $\begin{array}{l}e_{2} e_{1}=e_{3} \\
e_{3} e_{1}=e_{4}\end{array}$ & \\
\hline $\mathbf{D}_{03}^{4}(\lambda, \alpha)$ & : & $\begin{array}{l}e_{1} e_{1}=\lambda e_{3} \\
e_{2} e_{2}=e_{3}\end{array}$ & $\begin{array}{l}e_{1} e_{2}=e_{4} \\
e_{2} e_{3}=\alpha e_{4}\end{array}$ & $\begin{array}{l}e_{2} e_{1}=e_{3} \\
e_{3} e_{1}=e_{4}\end{array}$ & \\
\hline $\mathbf{D}_{04}^{4}(\lambda, \alpha)$ & : & $\begin{array}{l}e_{1} e_{1}=\lambda e_{3} \\
e_{2} e_{3}=\alpha e_{4}\end{array}$ & $\begin{array}{l}e_{2} e_{1}=e_{3} \\
e_{3} e_{1}=e_{4}\end{array}$ & $e_{2} e_{2}=e_{3}$ & \\
\hline $\mathbf{D}_{05}^{4}(\lambda, \alpha)$ & 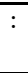 & $\begin{array}{l}e_{1} e_{1}=\lambda e_{3} \\
e_{2} e_{2}=e_{3}\end{array}$ & $\begin{array}{l}e_{1} e_{2}=\lambda e_{4} \\
e_{2} e_{3}=\Theta e_{4}\end{array}$ & $\begin{array}{l}e_{2} e_{1}=e_{3}+\lambda \alpha e_{4} \\
e_{3} e_{1}=\lambda e_{4}\end{array}$ & \\
\hline $\mathbf{D}_{06}^{4}(\lambda, \alpha)$ & : & $\begin{array}{l}e_{1} e_{1}=\lambda e_{3} \\
e_{2} e_{2}=e_{3}\end{array}$ & $\begin{array}{l}e_{1} e_{2}=e_{4} \\
e_{2} e_{3}=\Theta^{-1} e_{4}\end{array}$ & $\begin{array}{l}e_{2} e_{1}=e_{3}+\alpha e_{4} \\
e_{3} e_{1}=e_{4}\end{array}$ & \\
\hline
\end{tabular}




\begin{tabular}{|c|c|c|c|c|c|}
\hline $\mathbf{D}_{07}^{4}(\lambda)$ & : & $\begin{array}{l}e_{1} e_{1}=\lambda e_{3} \\
e_{2} e_{3}=\Theta e_{4}\end{array}$ & $\begin{array}{l}e_{2} e_{1}=e_{3}+\lambda e_{4} \\
e_{3} e_{1}=\lambda e_{4}\end{array}$ & $e_{2} e_{2}=e_{3}$ & \\
\hline $\mathbf{D}_{08}^{4}(\lambda)$ & $:$ & $\begin{array}{l}e_{1} e_{1}=\lambda e_{3} \\
e_{2} e_{3}=\Theta^{-1} e_{4}\end{array}$ & $\begin{array}{l}e_{2} e_{1}=e_{3}+e_{4} \\
e_{3} e_{1}=e_{4}\end{array}$ & $e_{2} e_{2}=e_{3}$ & \\
\hline $\mathbf{D}_{09}^{4}(\lambda, \alpha)$ & : & $\begin{array}{l}e_{1} e_{1}=\lambda e_{3} \\
e_{2} e_{1}=e_{3}\end{array}$ & $\begin{array}{l}e_{1} e_{2}=e_{4} \\
e_{2} e_{2}=e_{3}\end{array}$ & $\begin{array}{l}e_{1} e_{3}=\alpha e_{4} \\
e_{3} e_{1}=e_{4}\end{array}$ & \\
\hline $\mathbf{D}_{10}^{4}(\lambda, \alpha)$ & : & $\begin{array}{l}e_{1} e_{1}=\lambda e_{3} \\
e_{2} e_{2}=e_{3}\end{array}$ & $\begin{array}{l}e_{1} e_{3}=\alpha e_{4} \\
e_{3} e_{1}=e_{4}\end{array}$ & $e_{2} e_{1}=e_{3}$ & \\
\hline $\mathbf{D}_{11}^{4}(\lambda, \alpha)$ & : & $\begin{array}{l}e_{1} e_{1}=\lambda e_{3}+e_{4} \\
e_{2} e_{1}=e_{3}\end{array}$ & $\begin{array}{l}e_{1} e_{2}=\alpha e_{4} \\
e_{2} e_{2}=e_{3}\end{array}$ & $\begin{array}{l}e_{1} e_{3}=-e_{4} \\
e_{3} e_{1}=e_{4}\end{array}$ & \\
\hline $\mathbf{D}_{12}^{4}(\lambda, \alpha)$ & : & $\begin{array}{l}e_{1} e_{1}=\lambda e_{3}+e_{4} \\
e_{2} e_{2}=e_{3}\end{array}$ & $\begin{array}{l}e_{1} e_{3}=\alpha e_{4} \\
e_{3} e_{1}=\Theta e_{4}\end{array}$ & $\begin{array}{l}e_{2} e_{1}=e_{3} \\
e_{3} e_{2}=e_{4}\end{array}$ & \\
\hline $\mathbf{D}_{13}^{4}(\lambda, \alpha)$ & : & $\begin{array}{l}e_{1} e_{1}=\lambda e_{3}+e_{4} \\
e_{2} e_{2}=e_{3}\end{array}$ & $\begin{array}{l}e_{1} e_{3}=\alpha e_{4} \\
e_{3} e_{1}=(1-\Theta) e_{4}\end{array}$ & $\begin{array}{l}e_{2} e_{1}=e_{3} \\
e_{3} e_{2}=e_{4}\end{array}$ & \\
\hline $\mathbf{D}_{14}^{4}(\lambda, \alpha)$ & : & $\begin{array}{l}e_{1} e_{1}=\lambda e_{3} \\
e_{2} e_{2}=e_{3}\end{array}$ & $\begin{array}{l}e_{1} e_{3}=\alpha e_{4} \\
e_{3} e_{1}=\Theta e_{4}\end{array}$ & $\begin{array}{l}e_{2} e_{1}=e_{3} \\
e_{3} e_{2}=e_{4}\end{array}$ & \\
\hline $\mathbf{D}_{15}^{4}(\lambda, \alpha)$ & : & $\begin{array}{l}e_{1} e_{1}=\lambda e_{3} \\
e_{2} e_{2}=e_{3}\end{array}$ & $\begin{array}{l}e_{1} e_{3}=\alpha e_{4} \\
e_{3} e_{1}=(1-\Theta) e_{4}\end{array}$ & $\begin{array}{l}e_{2} e_{1}=e_{3} \\
e_{3} e_{2}=e_{4}\end{array}$ & \\
\hline $\mathbf{D}_{16}^{4}(\alpha)$ & : & $\begin{array}{l}e_{1} e_{3}=\alpha e_{4} \\
e_{3} e_{1}=e_{4}\end{array}$ & $\begin{array}{l}e_{2} e_{1}=e_{3}+e_{4} \\
e_{3} e_{2}=e_{4}\end{array}$ & $e_{2} e_{2}=e_{3}$ & \\
\hline $\mathbf{D}_{17}^{4}(\alpha)$ & : & $\begin{array}{l}e_{1} e_{1}=e_{4} \\
e_{2} e_{2}=e_{3}\end{array}$ & $\begin{array}{l}e_{1} e_{3}=-e_{4} \\
e_{3} e_{1}=e_{4}\end{array}$ & $\begin{array}{l}e_{2} e_{1}=e_{3}+\alpha e_{4} \\
e_{3} e_{2}=e_{4}\end{array}$ & \\
\hline $\mathbf{D}_{18}^{4}(\lambda, \alpha)$ & : & $\begin{array}{l}e_{1} e_{1}=\lambda e_{3}+e_{4} \\
e_{2} e_{3}=\alpha e_{4}\end{array}$ & $\begin{array}{l}e_{1} e_{3}=\Theta \alpha e_{4} \\
e_{3} e_{1}=\Theta e_{4}\end{array}$ & $\begin{array}{l}e_{2} e_{1}=e_{3} \\
e_{3} e_{2}=e_{4}\end{array}$ & $e_{2} e_{2}=e_{3}$ \\
\hline$\overline{\mathbf{D}_{19}^{4}(\lambda, \alpha)}$ & : & $\begin{array}{l}e_{1} e_{1}=\lambda e_{3}+e_{4} \\
e_{2} e_{3}=\alpha e_{4}\end{array}$ & $\begin{array}{l}e_{1} e_{3}=(1-\Theta) \alpha e_{4} \\
e_{3} e_{1}=(1-\Theta) e_{4}\end{array}$ & $\begin{array}{l}e_{2} e_{1}=e_{3} \\
e_{3} e_{2}=e_{4}\end{array}$ & $e_{2} e_{2}=e_{3}$ \\
\hline $\mathbf{D}_{20}^{4}(\lambda, \alpha)$ & : & $\begin{array}{l}e_{1} e_{1}=\lambda e_{3} \\
e_{2} e_{3}=\alpha e_{4}\end{array}$ & $\begin{array}{l}e_{1} e_{3}=\Theta \alpha e_{4} \\
e_{3} e_{1}=\Theta e_{4}\end{array}$ & $\begin{array}{l}e_{2} e_{1}=e_{3} \\
e_{3} e_{2}=e_{4}\end{array}$ & $e_{2} e_{2}=e_{3}$ \\
\hline $\mathbf{D}_{21}^{4}(\lambda, \alpha)$ & : & $\begin{array}{l}e_{1} e_{1}=\lambda e_{3} \\
e_{2} e_{3}=\alpha e_{4}\end{array}$ & $\begin{array}{l}e_{1} e_{3}=(1-\Theta) \alpha e_{4} \\
e_{3} e_{1}=(1-\Theta) e_{4}\end{array}$ & $\begin{array}{l}e_{2} e_{1}=e_{3} \\
e_{3} e_{2}=e_{4}\end{array}$ & $e_{2} e_{2}=e_{3}$ \\
\hline $\mathbf{D}_{22}^{4}(\lambda)$ & $:$ & $\begin{array}{l}e_{1} e_{1}=\lambda e_{3}+(1-2 \lambda) e_{4} \\
e_{2} e_{2}=e_{3}\end{array}$ & $\begin{array}{l}e_{1} e_{2}=e_{4} \\
e_{2} e_{3}=\left(1-\Theta^{-1}\right) e_{4}\end{array}$ & $\begin{array}{l}e_{1} e_{3}=(\Theta-1) e_{4} \\
e_{3} e_{1}=\Theta e_{4}\end{array}$ & $\begin{array}{l}e_{2} e_{1}=e_{3} \\
e_{3} e_{2}=e_{4}\end{array}$ \\
\hline $\mathbf{D}_{23}^{4}(\lambda)$ & : & $\begin{array}{l}e_{1} e_{1}=\lambda e_{3}+\lambda(1-2 \lambda) e_{4} \\
e_{2} e_{2}=e_{3}\end{array}$ & $\begin{array}{l}e_{1} e_{2}=\lambda e_{4} \\
e_{2} e_{3}=-\Theta^{2} e_{4}\end{array}$ & $\begin{array}{l}e_{1} e_{3}=-\lambda \Theta e_{4} \\
e_{3} e_{1}=\lambda(1-\Theta) e_{4}\end{array}$ & $\begin{array}{l}e_{2} e_{1}=e_{3} \\
e_{3} e_{2}=\lambda e_{4}\end{array}$ \\
\hline$\overline{\mathbf{D}_{24}^{4}(\lambda)}$ & : & $\begin{array}{l}e_{1} e_{1}=\lambda e_{3}+\Theta e_{4} \\
e_{2} e_{2}=e_{3}\end{array}$ & $\begin{array}{l}e_{1} e_{2}=e_{4} \\
e_{2} e_{3}=\left(1-\Theta^{-1}\right) e_{4}\end{array}$ & $\begin{array}{l}e_{1} e_{3}=(\Theta-1) e_{4} \\
e_{3} e_{1}=\Theta e_{4}\end{array}$ & $\begin{array}{l}e_{2} e_{1}=e_{3} \\
e_{3} e_{2}=e_{4}\end{array}$ \\
\hline $\mathbf{D}_{25}^{4}(\lambda)$ & : & $\begin{array}{l}e_{1} e_{1}=\lambda e_{3}+\lambda(1-\Theta) e_{4} \\
e_{2} e_{2}=e_{3}\end{array}$ & $\begin{array}{l}e_{1} e_{2}=\lambda e_{4} \\
e_{2} e_{3}=-\Theta^{2} e_{4}\end{array}$ & $\begin{array}{l}e_{1} e_{3}=-\lambda \Theta e_{4} \\
e_{3} e_{1}=\lambda(1-\Theta) e_{4}\end{array}$ & $\begin{array}{l}e_{2} e_{1}=e_{3} \\
e_{3} e_{2}=\lambda e_{4}\end{array}$ \\
\hline $\mathbf{D}_{26}^{4}(\lambda)$ & : & $\begin{array}{l}e_{1} e_{1}=\lambda e_{3}+\Theta e_{4} \\
e_{2} e_{2}=e_{3}\end{array}$ & $\begin{array}{l}e_{1} e_{2}=e_{4} \\
e_{2} e_{3}=-e_{4}\end{array}$ & $\begin{array}{l}e_{1} e_{3}=-\Theta e_{4} \\
e_{3} e_{1}=\Theta e_{4}\end{array}$ & $\begin{array}{l}e_{2} e_{1}=e_{3} \\
e_{3} e_{2}=e_{4}\end{array}$ \\
\hline $\mathbf{D}_{27}^{4}(\lambda)$ & : & $\begin{array}{l}e_{1} e_{1}=\lambda e_{3}+(1-\Theta) e_{4} \\
e_{2} e_{2}=e_{3}\end{array}$ & $\begin{array}{l}e_{1} e_{2}=e_{4} \\
e_{2} e_{3}=-e_{4}\end{array}$ & $\begin{array}{l}e_{1} e_{3}=(\Theta-1) e_{4} \\
e_{3} e_{1}=(1-\Theta) e_{4}\end{array}$ & $\begin{array}{l}e_{2} e_{1}=e_{3} \\
e_{3} e_{2}=e_{4}\end{array}$ \\
\hline $\mathbf{D}_{28}^{4}(\lambda)$ & $:$ & $\begin{array}{l}e_{1} e_{1}=\lambda e_{3}+(1-\Theta) e_{4} \\
e_{2} e_{2}=e_{3}\end{array}$ & $\begin{array}{l}e_{1} e_{2}=e_{4} \\
e_{2} e_{3}=-e_{4}\end{array}$ & $\begin{array}{l}e_{1} e_{3}=-\Theta e_{4} \\
e_{3} e_{1}=\Theta e_{4}\end{array}$ & $\begin{array}{l}e_{2} e_{1}=e_{3} \\
e_{3} e_{2}=e_{4}\end{array}$ \\
\hline$\overline{\mathbf{D}_{29}^{4}(\lambda)}$ & : & $\begin{array}{l}e_{1} e_{1}=\lambda e_{3}+\Theta e_{4} \\
e_{2} e_{2}=e_{3}\end{array}$ & $\begin{array}{l}e_{1} e_{2}=e_{4} \\
e_{2} e_{3}=-e_{4}\end{array}$ & $\begin{array}{l}e_{1} e_{3}=(\Theta-1) e_{4} \\
e_{3} e_{1}=(1-\Theta) e_{4}\end{array}$ & $\begin{array}{l}e_{2} e_{1}=e_{3} \\
e_{3} e_{2}=e_{4}\end{array}$ \\
\hline $\mathbf{D}_{30}^{4}(\lambda)$ & : & $\begin{array}{l}e_{1} e_{1}=\lambda e_{3}+e_{4} \\
e_{2} e_{3}=-e_{4}\end{array}$ & $\begin{array}{l}e_{1} e_{3}=(\Theta-1) e_{4} \\
e_{3} e_{1}=\Theta e_{4}\end{array}$ & $\begin{array}{l}e_{2} e_{1}=e_{3} \\
e_{3} e_{2}=e_{4}\end{array}$ & $e_{2} e_{2}=e_{3}$ \\
\hline $\mathbf{D}_{31}^{4}(\lambda)$ & $:$ & $\begin{array}{l}e_{1} e_{1}=\lambda e_{3}+e_{4} \\
e_{2} e_{3}=-e_{4}\end{array}$ & $\begin{array}{l}e_{1} e_{3}=-\Theta e_{4} \\
e_{3} e_{1}=(1-\Theta) e_{4}\end{array}$ & $\begin{array}{l}e_{2} e_{1}=e_{3} \\
e_{3} e_{2}=e_{4}\end{array}$ & $e_{2} e_{2}=e_{3}$ \\
\hline $\mathbf{D}_{32}^{4}(\lambda)$ & : & $\begin{array}{l}e_{1} e_{1}=\lambda e_{3} \\
e_{2} e_{3}=-e_{4}\end{array}$ & $\begin{array}{l}e_{1} e_{3}=(\Theta-1) e_{4} \\
e_{3} e_{1}=\Theta e_{4}\end{array}$ & $\begin{array}{l}e_{2} e_{1}=e_{3} \\
e_{3} e_{2}=e_{4}\end{array}$ & $e_{2} e_{2}=e_{3}$ \\
\hline $\mathbf{D}_{33}^{4}(\lambda)$ & : & $\begin{array}{l}e_{1} e_{1}=\lambda e_{3} \\
e_{2} e_{3}=-e_{4}\end{array}$ & $\begin{array}{l}e_{1} e_{3}=-\Theta e_{4} \\
e_{3} e_{1}=(1-\Theta) e_{4}\end{array}$ & $\begin{array}{l}e_{2} e_{1}=e_{3} \\
e_{3} e_{2}=e_{4}\end{array}$ & $e_{2} e_{2}=e_{3}$ \\
\hline
\end{tabular}




\begin{tabular}{|c|c|c|c|c|c|}
\hline & & $e_{3} e_{1}=e_{4}$ & $e_{3} e_{2}=e_{4}$ & & \\
\hline $\mathbf{D}_{35}^{4}(\lambda)$ & $:$ & $e_{1} e_{1}=\lambda e_{3}$ & $e_{1} e_{3}=e_{4}$ & $e_{2} e_{1}=e_{3}+e_{4}$ & $e_{2} e_{2}=e_{3}$ \\
\hline $\mathbf{D}_{36}^{4}(\lambda)$ & $:$ & $e_{1} e_{1}=\lambda e_{3}$ & $e_{1} e_{3}=e_{4}$ & $e_{2} e_{1}=e_{3}$ & $e_{2} e_{2}=e_{3}$ \\
\hline $\mathbf{D}_{37}^{4}(\lambda)$ & : & $\begin{array}{l}e_{1} e_{1}=\lambda e_{3}+e_{4} \\
e_{2} e_{2}=e_{3}\end{array}$ & $\begin{array}{l}e_{1} e_{3}=\Theta e_{4} \\
e_{2} e_{3}=e_{4}\end{array}$ & $e_{2} e_{1}=e_{3}$ & \\
\hline $\mathbf{D}_{38}^{4}(\lambda)$ & : & $\begin{array}{l}e_{1} e_{1}=\lambda e_{3}+e_{4} \\
e_{2} e_{2}=e_{3}\end{array}$ & $\begin{array}{l}e_{1} e_{3}=(1-\Theta) e_{4} \\
e_{2} e_{3}=e_{4}\end{array}$ & $e_{2} e_{1}=e_{3}$ & \\
\hline $\mathbf{D}_{39}^{4}(\lambda)$ & : & $\begin{array}{l}e_{1} e_{1}=\lambda e_{3} \\
e_{2} e_{2}=e_{3}\end{array}$ & $\begin{array}{l}e_{1} e_{3}=\Theta e_{4} \\
e_{2} e_{3}=e_{4}\end{array}$ & $e_{2} e_{1}=e_{3}$ & \\
\hline $\mathbf{D}_{40}^{4}(\lambda)$ & : & $\begin{array}{l}e_{1} e_{1}=\lambda e_{3} \\
e_{2} e_{2}=e_{3}\end{array}$ & $\begin{array}{l}e_{1} e_{3}=(1-\Theta) e_{4} \\
e_{2} e_{3}=e_{4}\end{array}$ & $e_{2} e_{1}=e_{3}$ & \\
\hline $\mathfrak{C D}_{01}^{4}$ & $:$ & $e_{1} e_{1}=e_{2}$ & $e_{2} e_{2}=e_{3}$ & & \\
\hline $\mathfrak{C} \mathfrak{D}_{02}^{4}$ & $:$ & $e_{1} e_{1}=e_{2}$ & $e_{2} e_{1}=e_{3}$ & $e_{2} e_{2}=e_{3}$ & \\
\hline $\mathfrak{C} \mathfrak{D}_{03}^{4}$ & $:$ & $e_{1} e_{1}=e_{2}$ & $e_{2} e_{1}=e_{3}$ & & \\
\hline $\mathfrak{C} \mathfrak{D}_{04}^{4}(\lambda)$ & $:$ & $e_{1} e_{1}=e_{2}$ & $e_{1} e_{2}=e_{3}$ & $e_{2} e_{1}=\lambda e_{3}$ & \\
\hline $\mathfrak{C} \mathfrak{D}_{05}^{4}$ & $:$ & $e_{1} e_{1}=e_{2}$ & $e_{2} e_{1}=e_{4}$ & $e_{2} e_{2}=e_{3}$ & \\
\hline $\mathfrak{C}^{2} \mathfrak{D}_{06}^{4}$ & $:$ & $e_{1} e_{1}=e_{2}$ & $e_{1} e_{2}=e_{4}$ & $e_{2} e_{1}=e_{3}$ & \\
\hline $\mathfrak{C} \mathfrak{D}_{07}^{4}(\lambda)$ & $:$ & $e_{1} e_{1}=e_{2}$ & $e_{1} e_{2}=e_{4}$ & $e_{2} e_{1}=\lambda e_{4}$ & $e_{2} e_{2}=e_{3}$ \\
\hline $\mathfrak{C} \mathfrak{D}_{08}^{4}(\alpha)$ & $:$ & $\begin{array}{l}e_{1} e_{1}=e_{2} \\
e_{2} e_{2}=\alpha e_{4}\end{array}$ & $\begin{array}{l}e_{1} e_{3}=e_{4} \\
e_{3} e_{1}=-2 e_{4}\end{array}$ & $e_{2} e_{1}=e_{3}$ & \\
\hline $\mathfrak{C} \mathfrak{D}_{09}^{4}$ & : & $\begin{array}{l}e_{1} e_{1}=e_{2} \\
e_{2} e_{1}=e_{3}\end{array}$ & $\begin{array}{l}e_{1} e_{2}=e_{4} \\
e_{2} e_{2}=-e_{4}\end{array}$ & $\begin{array}{l}e_{1} e_{3}=e_{4} \\
e_{3} e_{1}=-2 e_{4}\end{array}$ & \\
\hline $\mathfrak{C D}_{10}^{4}(\alpha)$ & : & $\begin{array}{l}e_{1} e_{1}=e_{2} \\
e_{2} e_{1}=e_{3}+e_{4}\end{array}$ & $\begin{array}{l}e_{1} e_{2}=e_{3} \\
e_{2} e_{2}=\alpha e_{4}\end{array}$ & $\begin{array}{l}e_{1} e_{3}=-e_{4} \\
e_{3} e_{1}=-e_{4}\end{array}$ & \\
\hline $\begin{array}{l}\mathfrak{C D}_{11}^{4}(\lambda) \\
\lambda \neq 1\end{array}$ & : & $\begin{array}{l}e_{1} e_{1}=e_{2} \\
e_{2} e_{1}=\lambda e_{3}+e_{4}\end{array}$ & $\begin{array}{l}e_{1} e_{2}=e_{3} \\
e_{2} e_{2}=-(\lambda+1)^{2} e_{4}\end{array}$ & $\begin{array}{l}e_{1} e_{3}=(\lambda-2) e_{4} \\
e_{3} e_{1}=(1-2 \lambda) e_{4}\end{array}$ & \\
\hline $\mathfrak{C} \mathfrak{D}_{12}^{4}(\alpha, \lambda)$ & $:$ & $\begin{array}{l}e_{1} e_{1}=e_{2} \\
e_{2} e_{1}=\lambda e_{3}\end{array}$ & $\begin{array}{l}e_{1} e_{2}=e_{3} \\
e_{2} e_{2}=\alpha e_{4}\end{array}$ & $\begin{array}{l}e_{1} e_{3}=(\lambda-2) e_{4} \\
e_{3} e_{1}=(1-2 \lambda) e_{4}\end{array}$ & \\
\hline $\begin{array}{l}\mathfrak{C D}_{13}^{4}(\alpha) \\
\alpha \neq \frac{1}{2}\end{array}$ & $:$ & $\begin{array}{l}e_{1} e_{1}=e_{2} \\
e_{2} e_{3}=\alpha e_{4}\end{array}$ & $\begin{array}{l}e_{1} e_{2}=e_{4} \\
e_{3} e_{1}=e_{4}\end{array}$ & $\begin{array}{l}e_{1} e_{3}=e_{4} \\
e_{3} e_{2}=(\alpha+1) e_{4}\end{array}$ & $e_{2} e_{1}=e_{4}$ \\
\hline $\mathfrak{C} \mathfrak{D}_{14}^{4}(\alpha, \beta)$ & : & $\begin{array}{l}e_{1} e_{1}=e_{2} \\
e_{2} e_{2}=e_{4}\end{array}$ & $\begin{array}{l}e_{1} e_{2}=e_{4} \\
e_{3} e_{1}=\alpha e_{4}\end{array}$ & $\begin{array}{l}e_{1} e_{3}=\alpha e_{4} \\
e_{3} e_{2}=e_{4}\end{array}$ & $\begin{array}{l}e_{2} e_{1}=e_{4} \\
e_{3} e_{3}=\beta e_{4}\end{array}$ \\
\hline $\mathfrak{C} \mathfrak{D}_{15}^{4}(\alpha)$ & : & $\begin{array}{l}e_{1} e_{1}=e_{2} \\
e_{2} e_{1}=(\alpha+1) e_{4}\end{array}$ & $\begin{array}{l}e_{1} e_{2}=\alpha e_{4} \\
e_{3} e_{1}=e_{4}\end{array}$ & $e_{1} e_{3}=e_{4}$ & \\
\hline $\mathfrak{C D}_{16}^{4}$ & : & $\begin{array}{l}e_{1} e_{1}=e_{2} \\
e_{2} e_{3}=-\frac{1}{2} e_{4}\end{array}$ & $\begin{array}{l}e_{1} e_{2}=e_{4} \\
e_{3} e_{2}=\frac{1}{2} e_{4}\end{array}$ & $\begin{array}{l}e_{2} e_{1}=e_{4} \\
e_{3} e_{3}=e_{4}\end{array}$ & \\
\hline $\mathfrak{C} \mathfrak{D}_{17}^{4}(\alpha)$ & : & $\begin{array}{l}e_{1} e_{1}=e_{2} \\
e_{2} e_{3}=\alpha e_{4}\end{array}$ & $\begin{array}{l}e_{1} e_{2}=e_{4} \\
e_{3} e_{2}=(\alpha+1) e_{4}\end{array}$ & $e_{2} e_{1}=e_{4}$ & \\
\hline $\mathfrak{C D}_{18}^{4}(\alpha)$ & $:$ & $e_{1} e_{1}=e_{2}$ & $e_{1} e_{2}=\alpha e_{4}$ & $e_{2} e_{1}=(\alpha+1) e_{4}$ & $e_{3} e_{3}=e_{4}$ \\
\hline $\mathfrak{C D}_{19}^{4}$ & : & $\begin{array}{l}e_{1} e_{1}=e_{2} \\
e_{3} e_{1}=e_{4}\end{array}$ & $\begin{array}{l}e_{1} e_{2}=e_{4} \\
e_{3} e_{3}=e_{4}\end{array}$ & $e_{2} e_{1}=e_{4}$ & \\
\hline $\mathfrak{C D}_{20}^{4}$ & $:$ & $e_{1} e_{1}=e_{2}$ & $e_{1} e_{2}=e_{4}$ & $e_{2} e_{1}=e_{4}$ & $e_{3} e_{1}=e_{4}$ \\
\hline $\mathfrak{C} \mathfrak{D}_{21}^{4}(\alpha)$ & $:$ & $\begin{array}{l}e_{1} e_{1}=e_{2} \\
e_{2} e_{3}=e_{4}\end{array}$ & $\begin{array}{l}e_{1} e_{3}=\alpha e_{4} \\
e_{3} e_{1}=\alpha e_{4}\end{array}$ & $\begin{array}{l}e_{2} e_{1}=e_{4} \\
e_{3} e_{2}=e_{4}\end{array}$ & $\begin{array}{l}e_{2} e_{2}=e_{4} \\
e_{3} e_{3}=e_{4}\end{array}$ \\
\hline $\mathfrak{C D}_{22}^{4}$ & : & $\begin{array}{l}e_{1} e_{1}=e_{2} \\
e_{3} e_{1}=e_{4}\end{array}$ & $\begin{array}{l}e_{1} e_{3}=e_{4} \\
e_{3} e_{2}=\frac{1}{2} e_{4}\end{array}$ & $\begin{array}{l}e_{2} e_{3}=-\frac{1}{2} e_{4} \\
e_{3} e_{3}=e_{4}\end{array}$ & \\
\hline $\mathfrak{C} \mathfrak{D}_{23}^{4}(\alpha)$ & : & $\begin{array}{l}e_{1} e_{1}=e_{2} \\
e_{3} e_{1}=e_{4}\end{array}$ & $\begin{array}{l}e_{1} e_{3}=e_{4} \\
e_{3} e_{2}=(\alpha+1) e_{4}\end{array}$ & $e_{2} e_{3}=\alpha e_{4}$ & \\
\hline $\mathfrak{C} \mathfrak{D}_{24}^{4}(\alpha)$ & : & $\begin{array}{l}e_{1} e_{1}=e_{2} \\
e_{3} e_{1}=e_{4}\end{array}$ & $\begin{array}{l}e_{1} e_{3}=e_{4} \\
e_{3} e_{2}=e_{4}\end{array}$ & $\begin{array}{l}e_{2} e_{2}=e_{4} \\
e_{3} e_{3}=\alpha e_{4}\end{array}$ & \\
\hline $\mathfrak{C} \mathfrak{D}_{25}^{4}$ & : & $\begin{array}{l}e_{1} e_{1}=e_{2} \\
e_{3} e_{1}=e_{4}\end{array}$ & $\begin{array}{l}e_{1} e_{3}=e_{4} \\
e_{2} e_{2}=e_{4}\end{array}$ & $e_{2} e_{1}=e_{4}$ & \\
\hline $\mathfrak{C D}_{26}^{4}(\alpha)$ & $:$ & $e_{1} e_{1}=e_{2}$ & $e_{1} e_{3}=\alpha e_{4}$ & $e_{2} e_{2}=e_{4}$ & $e_{3} e_{1}=(\alpha+1) e_{4}$ \\
\hline $\mathfrak{C} \mathfrak{D}_{27}^{4}$ & : & $\begin{array}{l}e_{1} e_{1}=e_{2} \\
e_{3} e_{2}=e_{4}\end{array}$ & $\begin{array}{l}e_{2} e_{1}=e_{4} \\
e_{3} e_{3}=e_{4}\end{array}$ & $e_{2} e_{3}=e_{4}$ & \\
\hline
\end{tabular}




\begin{tabular}{|c|c|c|c|c|c|}
\hline $\begin{array}{l}\mathfrak{C D}_{28}^{4}(\alpha) \\
\alpha \neq 1\end{array}$ & $:$ & $\begin{array}{l}e_{1} e_{1}=e_{2} \\
e_{2} e_{3}=e_{4}\end{array}$ & $\begin{array}{l}e_{2} e_{1}=e_{4} \\
e_{3} e_{2}=e_{4}\end{array}$ & $\begin{array}{l}e_{2} e_{2}=e_{4} \\
e_{3} e_{3}=\alpha e_{4}\end{array}$ & \\
\hline $\mathfrak{C D}_{29}^{4}$ & $:$ & $e_{1} e_{1}=e_{2}$ & $e_{2} e_{3}=-\frac{1}{2} e_{4}$ & $e_{3} e_{2}=\frac{1}{2} e_{4}$ & $e_{3} e_{3}=e_{4}$ \\
\hline $\mathfrak{C} \mathfrak{D}_{30}^{4}$ & $:$ & $e_{1} e_{1}=e_{2}$ & $e_{2} e_{1}=e_{4}$ & $e_{2} e_{3}=e_{4}$ & $e_{3} e_{2}=e_{4}$ \\
\hline $\mathfrak{C D}_{31}^{4}$ & $:$ & $e_{1} e_{1}=e_{2}$ & $e_{2} e_{3}=e_{4}$ & $e_{3} e_{1}=e_{4}$ & $e_{3} e_{2}=e_{4}$ \\
\hline $\mathfrak{C} \mathfrak{D}_{32}^{4}(\alpha)$ & $:$ & $e_{1} e_{1}=e_{2}$ & $e_{2} e_{3}=\alpha e_{4}$ & $e_{3} e_{2}=(\alpha+1) e_{4}$ & \\
\hline $\mathfrak{C D}_{33}^{4}$ & $:$ & $e_{1} e_{1}=e_{2}$ & $e_{2} e_{1}=e_{4}$ & $e_{2} e_{2}=e_{4}$ & $e_{3} e_{3}=e_{4}$ \\
\hline $\mathfrak{C} \mathfrak{D}_{34}^{4}$ & $:$ & $e_{1} e_{1}=e_{2}$ & $e_{2} e_{2}=e_{4}$ & $e_{3} e_{3}=e_{4}$ & \\
\hline $\mathfrak{C} \mathfrak{D}_{35}^{4}$ & $:$ & $e_{1} e_{1}=e_{2}$ & $e_{2} e_{2}=e_{4}$ & $e_{3} e_{1}=e_{4}$ & $e_{3} e_{3}=e_{4}$ \\
\hline $\mathfrak{C} \mathfrak{D}_{36}^{4}(\alpha)$ & $:$ & $e_{1} e_{1}=e_{2}$ & $e_{2} e_{2}=e_{4}$ & $e_{3} e_{2}=e_{4}$ & $e_{3} e_{3}=\alpha e_{4}$ \\
\hline $\mathfrak{C} \mathfrak{D}_{37}^{4}$ & $:$ & $e_{1} e_{1}=e_{2}$ & $e_{1} e_{2}=e_{4}$ & $e_{2} e_{1}=e_{4}$ & $e_{3} e_{3}=e_{4}$ \\
\hline $\mathfrak{C} \mathfrak{D}_{38}^{4}$ & $:$ & $e_{1} e_{1}=e_{2}$ & $e_{2} e_{3}=e_{4}$ & $e_{3} e_{2}=e_{4}$ & \\
\hline $\mathfrak{C D}_{39}^{4}$ & : & $\begin{array}{l}e_{1} e_{1}=e_{3}+e_{4} \\
e_{2} e_{2}=e_{3}\end{array}$ & $\begin{array}{l}e_{1} e_{2}=\frac{\imath}{2} e_{4} \\
e_{2} e_{3}=-2 i e_{4}\end{array}$ & $\begin{array}{l}e_{1} e_{3}=e_{4} \\
e_{3} e_{1}=2 e_{4}\end{array}$ & $\begin{array}{l}e_{2} e_{1}=\frac{\imath}{2} e_{4} \\
e_{3} e_{2}=-i e_{4}\end{array}$ \\
\hline $\mathfrak{C D}_{40}^{4}$ & $:$ & $\begin{array}{l}e_{1} e_{1}=e_{3}+e_{4} \\
e_{2} e_{2}=e_{3}\end{array}$ & $\begin{array}{l}e_{1} e_{2}=\frac{i}{2} e_{4} \\
e_{2} e_{3}=-\frac{i}{2} e_{4}\end{array}$ & $\begin{array}{l}e_{1} e_{3}=-\frac{1}{2} e_{4} \\
e_{3} e_{1}=\frac{1}{2} e_{4}\end{array}$ & $\begin{array}{l}e_{2} e_{1}=\frac{i}{2} e_{4} \\
e_{3} e_{2}=\frac{i}{2} e_{4}\end{array}$ \\
\hline $\mathfrak{C} \mathfrak{D}_{41}^{4}$ & : & $\begin{array}{l}e_{1} e_{1}=e_{3}+e_{4} \\
e_{2} e_{2}=e_{3}\end{array}$ & $\begin{array}{l}e_{1} e_{2}=-\frac{\imath}{2} e_{4} \\
e_{2} e_{3}=-2 i e_{4}\end{array}$ & $\begin{array}{l}e_{1} e_{3}=e_{4} \\
e_{3} e_{1}=2 e_{4}\end{array}$ & $\begin{array}{l}e_{2} e_{1}=-\frac{\imath}{2} e_{4} \\
e_{3} e_{2}=-i e_{4}\end{array}$ \\
\hline $\mathfrak{C} \mathfrak{D}_{42}^{4}$ & : & $\begin{array}{l}e_{1} e_{1}=e_{3}+e_{4} \\
e_{2} e_{2}=e_{3}\end{array}$ & $\begin{array}{l}e_{1} e_{2}=-\frac{i}{2} e_{4} \\
e_{2} e_{3}=-\frac{i}{2} e_{4}\end{array}$ & $\begin{array}{l}e_{1} e_{3}=-\frac{1}{2} e_{4} \\
e_{3} e_{1}=\frac{1}{2} e_{4}\end{array}$ & $\begin{array}{l}e_{2} e_{1}=-\frac{i}{2} e_{4} \\
e_{3} e_{2}=\frac{i}{2} e_{4}\end{array}$ \\
\hline $\mathfrak{C} \mathfrak{D}_{43}^{4}(\alpha)$ & $:$ & $\begin{array}{l}e_{1} e_{1}=e_{3}+e_{4} \\
e_{2} e_{1}=\alpha e_{4}\end{array}$ & $\begin{array}{l}e_{1} e_{2}=\alpha e_{4} \\
e_{2} e_{2}=e_{3}\end{array}$ & $\begin{array}{l}e_{1} e_{3}=-\frac{1}{2} e_{4} \\
e_{3} e_{1}=\frac{1}{2} e_{4}\end{array}$ & \\
\hline $\mathfrak{C} \mathfrak{D}_{44}^{4}(\alpha, \beta, \gamma)$ & : & $\begin{array}{l}e_{1} e_{1}=e_{3}+\alpha e_{4} \\
e_{2} e_{2}=e_{3}\end{array}$ & $\begin{array}{l}e_{1} e_{2}=\beta e_{4} \\
e_{3} e_{1}=e_{4}\end{array}$ & $\begin{array}{l}e_{2} e_{1}=(\beta+\gamma) e_{4} \\
e_{3} e_{3}=e_{4}\end{array}$ & \\
\hline $\mathfrak{C D}_{45}^{4}$ & : & $\begin{array}{l}e_{1} e_{1}=e_{3}+2 i e_{4} \\
e_{3} e_{1}=e_{4}\end{array}$ & $\begin{array}{l}e_{1} e_{2}=e_{4} \\
e_{3} e_{2}=i e_{4}\end{array}$ & $\begin{array}{l}e_{2} e_{1}=e_{4} \\
e_{3} e_{3}=e_{4}\end{array}$ & $e_{2} e_{2}=e_{3}$ \\
\hline $\begin{array}{l}\mathfrak{C D}_{46}^{4}(\alpha) \\
\alpha \neq 0\end{array}$ & : & $\begin{array}{l}e_{1} e_{1}=e_{3}-2 i \alpha e_{4} \\
e_{3} e_{1}=e_{4}\end{array}$ & $\begin{array}{l}e_{1} e_{2}=\alpha e_{4} \\
e_{3} e_{2}=i e_{4}\end{array}$ & $\begin{array}{l}e_{2} e_{1}=\alpha e_{4} \\
e_{3} e_{3}=e_{4}\end{array}$ & $e_{2} e_{2}=e_{3}$ \\
\hline $\begin{array}{l}\mathfrak{C D}_{47}^{4}(\alpha, \beta) \\
\beta \neq 0\end{array}$ & : & $\begin{array}{l}e_{1} e_{1}=e_{3}+e_{4} \\
e_{2} e_{3}=\beta e_{4}\end{array}$ & $\begin{array}{l}e_{1} e_{3}=\alpha e_{4} \\
e_{3} e_{1}=(\alpha+1) e_{4}\end{array}$ & $\begin{array}{l}e_{2} e_{2}=e_{3} \\
e_{3} e_{2}=\beta e_{4}\end{array}$ & \\
\hline $\begin{array}{l}\mathfrak{C D}_{48}^{4}(\alpha) \\
\alpha \neq 0\end{array}$ & $:$ & $\begin{array}{l}e_{1} e_{1}=e_{3}+\alpha e_{4} \\
e_{3} e_{1}=e_{4}\end{array}$ & $\begin{array}{l}e_{2} e_{1}=i \alpha e_{4} \\
e_{3} e_{2}=i e_{4}\end{array}$ & $\begin{array}{l}e_{2} e_{2}=e_{3} \\
e_{3} e_{3}=e_{4}\end{array}$ & \\
\hline $\begin{array}{l}\mathfrak{C D}_{49}^{4}(\alpha) \\
\alpha \neq 0\end{array}$ & $:$ & $\begin{array}{l}e_{1} e_{1}=e_{3}+\alpha e_{4} \\
e_{3} e_{1}=e_{4}\end{array}$ & $\begin{array}{l}e_{2} e_{1}=-i \alpha e_{4} \\
e_{3} e_{2}=i e_{4}\end{array}$ & $\begin{array}{l}e_{2} e_{2}=e_{3} \\
e_{3} e_{3}=e_{4}\end{array}$ & \\
\hline $\mathfrak{C} \mathfrak{D}_{50}^{4}(\alpha)$ & $:$ & $e_{1} e_{1}=e_{3}+\alpha e_{4}$ & $e_{2} e_{1}=e_{4}$ & $e_{2} e_{2}=e_{3}$ & $e_{3} e_{3}=e_{4}$ \\
\hline $\mathfrak{C D}_{51}^{4}(\alpha)$ & : & $\begin{array}{l}e_{1} e_{1}=e_{3}+\alpha e_{4} \\
e_{3} e_{2}=i e_{4}\end{array}$ & $\begin{array}{l}e_{2} e_{2}=e_{3} \\
e_{3} e_{3}=e_{4}\end{array}$ & $e_{3} e_{1}=e_{4}$ & \\
\hline $\mathfrak{C} \mathfrak{D}_{52}^{4}$ & $:$ & $e_{1} e_{1}=e_{3}+e_{4}$ & $e_{2} e_{2}=e_{3}$ & $e_{3} e_{3}=e_{4}$ & \\
\hline $\mathfrak{C} \mathfrak{D}_{53}^{4}$ & $:$ & $\begin{array}{l}e_{1} e_{1}=e_{3} \\
e_{2} e_{2}=e_{3}\end{array}$ & $\begin{array}{l}e_{1} e_{2}=-\frac{1}{2} e_{4} \\
e_{2} e_{3}=i e_{4}\end{array}$ & $\begin{array}{l}e_{1} e_{3}=e_{4} \\
e_{3} e_{1}=e_{4}\end{array}$ & $\begin{array}{l}e_{2} e_{1}=\frac{1}{2} e_{4} \\
e_{3} e_{2}=i e_{4}\end{array}$ \\
\hline $\mathfrak{C} \mathfrak{D}_{54}^{4}(\alpha)$ & 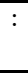 & $\begin{array}{l}e_{1} e_{1}=e_{3} \\
e_{2} e_{2}=e_{3}\end{array}$ & $\begin{array}{l}e_{1} e_{2}=e_{4} \\
e_{2} e_{3}=-i(\alpha+1) e_{4}\end{array}$ & $\begin{array}{l}e_{1} e_{3}=\alpha e_{4} \\
e_{3} e_{1}=(\alpha+1) e_{4}\end{array}$ & $\begin{array}{l}e_{2} e_{1}=e_{4} \\
e_{3} e_{2}=-i \alpha e_{4}\end{array}$ \\
\hline $\mathfrak{C} \mathfrak{D}_{55}^{4}(\alpha)$ & : & $\begin{array}{l}e_{1} e_{1}=e_{3} \\
e_{2} e_{1}=e_{4}\end{array}$ & $\begin{array}{l}e_{1} e_{2}=e_{4} \\
e_{2} e_{2}=e_{3}\end{array}$ & $\begin{array}{l}e_{1} e_{3}=\alpha e_{4} \\
e_{3} e_{1}=(\alpha+1) e_{4}\end{array}$ & \\
\hline $\mathfrak{C} \mathfrak{D}_{56}^{4}$ & $:$ & $\begin{array}{l}e_{1} e_{1}=e_{3} \\
e_{3} e_{1}=e_{4}\end{array}$ & $\begin{array}{l}e_{1} e_{2}=e_{4} \\
e_{3} e_{2}=i e_{4}\end{array}$ & $\begin{array}{l}e_{2} e_{1}=-e_{4} \\
e_{3} e_{3}=e_{4}\end{array}$ & $e_{2} e_{2}=e_{3}$ \\
\hline $\begin{array}{l}\mathfrak{C}_{57}^{4}(\alpha, \beta) \\
\beta \notin\{0,-2 \alpha\}\end{array}$ & : & $\begin{array}{l}e_{1} e_{1}=e_{3} \\
e_{3} e_{1}=e_{4}\end{array}$ & $\begin{array}{l}e_{1} e_{2}=\alpha e_{4} \\
e_{3} e_{2}=i e_{4}\end{array}$ & $\begin{array}{l}e_{2} e_{1}=(\alpha+\beta) e_{4} \\
e_{3} e_{3}=e_{4}\end{array}$ & $e_{2} e_{2}=e_{3}$ \\
\hline $\mathfrak{C D}_{58}^{4}$ & $:$ & $\begin{array}{l}e_{1} e_{1}=e_{3} \\
e_{2} e_{3}=i e_{4}\end{array}$ & $\begin{array}{l}e_{1} e_{3}=e_{4} \\
e_{3} e_{1}=e_{4}\end{array}$ & $\begin{array}{l}e_{2} e_{1}=e_{4} \\
e_{3} e_{2}=i e_{4}\end{array}$ & $e_{2} e_{2}=e_{3}$ \\
\hline $\begin{array}{l}\mathfrak{C D}_{59}^{4}(\alpha, \beta) \\
\beta \neq 0\end{array}$ & : & $\begin{array}{l}e_{1} e_{1}=e_{3} \\
e_{2} e_{3}=\beta e_{4}\end{array}$ & $\begin{array}{l}e_{1} e_{3}=\alpha e_{4} \\
e_{3} e_{1}=(\alpha+1) e_{4}\end{array}$ & $\begin{array}{l}e_{2} e_{2}=e_{3} \\
e_{3} e_{2}=\beta e_{4}\end{array}$ & \\
\hline $\mathfrak{C D}_{60}^{4}$ & $:$ & $\begin{array}{l}e_{1} e_{1}=e_{3} \\
e_{2} e_{3}=e_{4}\end{array}$ & $\begin{array}{l}e_{1} e_{3}=i e_{4} \\
e_{3} e_{1}=(i+1) e_{4}\end{array}$ & $\begin{array}{l}e_{2} e_{2}=e_{3} \\
e_{3} e_{2}=(i+1) e_{4}\end{array}$ & \\
\hline
\end{tabular}




\begin{tabular}{|c|c|c|c|c|c|}
\hline $\mathfrak{C D}_{61}^{4}(\alpha)$ & $:$ & $\begin{array}{l}e_{1} e_{1}=e_{3} \\
e_{2} e_{3}=\alpha e_{4}\end{array}$ & $\begin{array}{l}e_{1} e_{3}=-i \alpha e_{4} \\
e_{3} e_{1}=(1-i \alpha) e_{4}\end{array}$ & $\begin{array}{l}e_{2} e_{2}=e_{3} \\
e_{3} e_{2}=(\alpha+i) e_{4}\end{array}$ & \\
\hline $\mathfrak{C D}_{62}^{4}$ & : & $\begin{array}{l}e_{1} e_{1}=e_{3} \\
e_{2} e_{3}=-2 i e_{4}\end{array}$ & $\begin{array}{l}e_{1} e_{3}=e_{4} \\
e_{3} e_{1}=2 e_{4}\end{array}$ & $\begin{array}{l}e_{2} e_{2}=e_{3} \\
e_{3} e_{2}=-i e_{4}\end{array}$ & \\
\hline $\mathfrak{C D}_{63}^{4}$ & : & $\begin{aligned} e_{1} e_{1} & =e_{3} \\
e_{2} e_{3} & =-\frac{i}{2} e_{4}\end{aligned}$ & $\begin{array}{l}e_{1} e_{3}=-\frac{1}{2} e_{4} \\
e_{3} e_{1}=\frac{1}{2} e_{4}\end{array}$ & $\begin{array}{l}e_{2} e_{2}=e_{3} \\
e_{3} e_{2}=\frac{i}{2} e_{4}\end{array}$ & \\
\hline $\mathfrak{C D}_{64}^{4}(\alpha)$ & $:$ & $e_{1} e_{1}=e_{3}$ & $e_{1} e_{3}=\alpha e_{4}$ & $e_{2} e_{2}=e_{3}$ & $e_{3} e_{1}=(\alpha+1) e_{4}$ \\
\hline $\begin{array}{l}\mathfrak{C D}_{65}^{4}(\alpha) \\
\alpha \neq 0\end{array}$ & $:$ & $\begin{array}{l}e_{1} e_{1}=e_{3} \\
e_{3} e_{1}=(\alpha+1) e_{4}\end{array}$ & $\begin{array}{l}e_{1} e_{3}=\alpha e_{4} \\
e_{3} e_{2}=i e_{4}\end{array}$ & $e_{2} e_{2}=e_{3}$ & \\
\hline $\mathfrak{C D}_{66}^{4}$ & : & $\begin{array}{l}e_{1} e_{1}=e_{3} \\
e_{2} e_{3}=e_{4}\end{array}$ & $\begin{array}{l}e_{2} e_{1}=e_{4} \\
e_{3} e_{2}=e_{4}\end{array}$ & $e_{2} e_{2}=e_{3}$ & \\
\hline $\mathfrak{C D}_{67}^{4}$ & $:$ & $e_{1} e_{1}=e_{3}$ & $e_{2} e_{2}=e_{3}$ & $e_{3} e_{3}=e_{4}$ & \\
\hline $\mathfrak{C D}_{68}^{4}$ & : & $\begin{array}{l}e_{1} e_{1}=e_{3}+e_{4} \\
e_{2} e_{3}=e_{4}\end{array}$ & $\begin{array}{l}e_{1} e_{3}=i e_{4} \\
e_{3} e_{1}=i e_{4}\end{array}$ & $\begin{array}{l}e_{2} e_{2}=e_{3} \\
e_{3} e_{2}=e_{4}\end{array}$ & \\
\hline $\mathfrak{C D}_{69}^{4}$ & : & $\begin{array}{l}e_{1} e_{1}=e_{3} \\
e_{2} e_{3}=e_{4}\end{array}$ & $\begin{array}{l}e_{1} e_{3}=i e_{4} \\
e_{3} e_{1}=i e_{4}\end{array}$ & $\begin{array}{l}e_{2} e_{2}=e_{3} \\
e_{3} e_{2}=e_{4}\end{array}$ & \\
\hline $\mathfrak{C} \mathfrak{D}_{70}^{4}$ & $:$ & $e_{1} e_{1}=e_{3}$ & $e_{1} e_{3}=e_{4}$ & $e_{2} e_{2}=e_{3}$ & $e_{3} e_{1}=e_{4}$ \\
\hline $\mathfrak{C} \mathfrak{D}_{71}^{4}$ & : & $\begin{array}{l}e_{1} e_{1}=e_{4} \\
e_{2} e_{1}=-e_{3}\end{array}$ & $\begin{array}{l}e_{1} e_{2}=e_{3} \\
e_{3} e_{1}=-e_{4}\end{array}$ & $e_{1} e_{3}=e_{4}$ & \\
\hline $\mathfrak{C D}_{72}^{4}$ & $:$ & $\begin{array}{l}e_{1} e_{1}=e_{4} \\
e_{2} e_{1}=-e_{3}\end{array}$ & $\begin{array}{l}e_{1} e_{2}=e_{3} \\
e_{2} e_{2}=e_{4}\end{array}$ & $\begin{array}{l}e_{1} e_{3}=e_{4} \\
e_{3} e_{1}=-e_{4}\end{array}$ & \\
\hline $\mathfrak{C D}_{73}^{4}$ & $:$ & $e_{1} e_{2}=e_{3}+e_{4}$ & $e_{1} e_{3}=e_{4}$ & $e_{2} e_{1}=-e_{3}$ & $e_{3} e_{1}=-e_{4}$ \\
\hline $\mathfrak{C D}_{74}^{4}(\alpha)$ & $:$ & $e_{1} e_{2}=e_{3}$ & $e_{1} e_{3}=(\alpha+1) e_{4}$ & $e_{2} e_{1}=-e_{3}$ & $e_{3} e_{1}=-\alpha e_{4}$ \\
\hline $\mathfrak{C} \mathfrak{D}_{75}^{4}(\alpha)$ & $:$ & $\begin{array}{l}e_{1} e_{2}=e_{3} \\
e_{2} e_{2}=e_{4}\end{array}$ & $\begin{array}{l}e_{1} e_{3}=(\alpha+1) e_{4} \\
e_{3} e_{1}=-\alpha e_{4}\end{array}$ & $e_{2} e_{1}=-e_{3}$ & \\
\hline $\mathfrak{C D}_{76}^{4}$ & 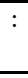 & $\begin{array}{l}e_{1} e_{2}=e_{3} \\
e_{2} e_{2}=e_{4}\end{array}$ & $\begin{array}{l}e_{1} e_{3}=e_{4} \\
e_{3} e_{1}=-e_{4}\end{array}$ & $e_{2} e_{1}=-e_{3}$ & \\
\hline $\mathfrak{C D}_{77}^{4}$ & : & $\begin{array}{l}e_{1} e_{2}=e_{3} \\
e_{2} e_{3}=e_{4}\end{array}$ & $\begin{array}{l}e_{1} e_{3}=e_{4} \\
e_{3} e_{2}=-e_{4}\end{array}$ & $e_{2} e_{1}=-e_{3}$ & \\
\hline $\mathfrak{C D}_{78}^{4}$ & : & $\begin{array}{l}e_{1} e_{2}=e_{3} \\
e_{2} e_{2}=e_{4}\end{array}$ & $\begin{array}{l}e_{1} e_{3}=e_{4} \\
e_{2} e_{3}=e_{4}\end{array}$ & $\begin{array}{l}e_{2} e_{1}=-e_{3} \\
e_{3} e_{2}=-e_{4}\end{array}$ & \\
\hline $\mathfrak{C} \mathfrak{D}_{79}^{4}(\alpha)$ & 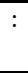 & $\begin{array}{l}e_{1} e_{2}=e_{3}+\alpha e_{4} \\
e_{2} e_{3}=e_{4}\end{array}$ & $\begin{array}{l}e_{1} e_{3}=e_{4} \\
e_{3} e_{3}=e_{4}\end{array}$ & $e_{2} e_{1}=-e_{3}$ & \\
\hline $\mathfrak{C D}_{80}^{4}$ & $:$ & $e_{1} e_{2}=e_{3}+e_{4}$ & $e_{1} e_{3}=e_{4}$ & $e_{2} e_{1}=-e_{3}$ & $e_{3} e_{3}=e_{4}$ \\
\hline $\mathfrak{C D}_{81}^{4}$ & $:$ & $e_{1} e_{2}=e_{3}+e_{4}$ & $e_{2} e_{1}=-e_{3}$ & $e_{3} e_{3}=e_{4}$ & \\
\hline $\mathfrak{C D}_{82}^{4}$ & $:$ & $\begin{array}{l}e_{1} e_{2}=e_{3} \\
e_{2} e_{2}=e_{4}\end{array}$ & $\begin{array}{l}e_{1} e_{3}=e_{4} \\
e_{3} e_{3}=e_{4}\end{array}$ & $e_{2} e_{1}=-e_{3}$ & \\
\hline $\begin{array}{l}\mathfrak{C D}_{83}^{4}(\alpha) \\
\alpha \neq 0\end{array}$ & 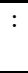 & $\begin{array}{l}e_{1} e_{2}=e_{3} \\
e_{2} e_{3}=e_{4}\end{array}$ & $\begin{array}{l}e_{2} e_{1}=-e_{3} \\
e_{3} e_{3}=e_{4}\end{array}$ & $e_{2} e_{2}=\alpha e_{4}$ & \\
\hline $\mathfrak{C D}_{84}^{4}$ & $:$ & $e_{1} e_{2}=e_{3}$ & $e_{2} e_{1}=-e_{3}$ & $e_{2} e_{2}=e_{4}$ & $e_{3} e_{3}=e_{4}$ \\
\hline $\mathfrak{C} \mathfrak{D}_{85}^{4}$ & $:$ & $e_{1} e_{2}=e_{3}$ & $e_{2} e_{1}=-e_{3}$ & $e_{3} e_{3}=e_{4}$ & \\
\hline $\mathfrak{C} \mathfrak{D}_{86}^{4}$ & $:$ & $e_{1} e_{2}=e_{3}$ & $e_{2} e_{1}=-e_{3}$ & $e_{2} e_{3}=e_{4}$ & $e_{3} e_{2}=-e_{4}$ \\
\hline $\begin{array}{l}\mathfrak{C D}_{87}^{4}(\lambda) \\
\lambda \neq 0, \frac{1}{4}\end{array}$ & $:$ & $\begin{array}{l}e_{1} e_{1}=\lambda e_{3}+(2 \Theta-1) e_{4} \\
e_{2} e_{2}=e_{3}\end{array}$ & $\begin{array}{l}e_{1} e_{2}=e_{4} \\
e_{2} e_{3}=\Theta \lambda^{-1} e_{4}\end{array}$ & $\begin{array}{l}e_{1} e_{3}=e_{4} \\
e_{3} e_{3}=e_{4}\end{array}$ & $e_{2} e_{1}=e_{3}-(1-\Theta)^{2} \lambda^{-1} e_{4}$ \\
\hline $\begin{array}{l}\mathfrak{C D}_{88}^{4}(\lambda) \\
\lambda \neq 0, \frac{1}{4}\end{array}$ & $:$ & $\begin{array}{l}e_{1} e_{1}=\lambda e_{3}+(1-2 \Theta) e_{4} \\
e_{2} e_{2}=e_{3}\end{array}$ & $\begin{array}{l}e_{1} e_{2}=e_{4} \\
e_{2} e_{3}=\Theta \lambda^{-1} e_{4}\end{array}$ & $\begin{array}{l}e_{1} e_{3}=e_{4} \\
e_{3} e_{3}=e_{4}\end{array}$ & $e_{2} e_{1}=e_{3}-\Theta^{2} \lambda^{-1} e_{4}$ \\
\hline $\begin{array}{l}\mathfrak{C D}_{89}^{4}(\lambda) \\
\lambda \neq 0, \frac{1}{4}\end{array}$ & $:$ & $\begin{array}{l}e_{1} e_{1}=\lambda e_{3}+(2 \Theta-1) e_{4} \\
e_{2} e_{2}=e_{3}\end{array}$ & $\begin{array}{l}e_{1} e_{2}=e_{4} \\
e_{2} e_{3}=(1-\Theta) \lambda^{-1} e_{4}\end{array}$ & $\begin{array}{l}e_{1} e_{3}=e_{4} \\
e_{3} e_{3}=e_{4}\end{array}$ & $e_{2} e_{1}=e_{3}-(1-\Theta)^{2} \lambda^{-1} e_{4}$ \\
\hline $\begin{array}{l}\mathfrak{C D}_{90}^{4}(\lambda) \\
\lambda \neq 0, \frac{1}{4}\end{array}$ & $:$ & $\begin{array}{l}e_{1} e_{1}=\lambda e_{3}+(1-2 \Theta) e_{4} \\
e_{2} e_{2}=e_{3}\end{array}$ & $\begin{array}{l}e_{1} e_{2}=e_{4} \\
e_{2} e_{3}=(1-\Theta) \lambda^{-1} e_{4}\end{array}$ & $\begin{array}{l}e_{1} e_{3}=e_{4} \\
e_{3} e_{3}=e_{4}\end{array}$ & $e_{2} e_{1}=e_{3}-\Theta^{2} \lambda^{-1} e_{4}$ \\
\hline $\begin{array}{l}\mathfrak{C D}_{91}^{4}(\lambda, \alpha) \\
\lambda \neq 0, \frac{1}{4}\end{array}$ & : & $\begin{array}{l}e_{1} e_{1}=\lambda e_{3}+(2 \Theta-1) e_{4} \\
e_{2} e_{1}=e_{3}-(1-\Theta)^{2} \lambda^{-1} e_{4}\end{array}$ & $\begin{array}{l}e_{1} e_{2}=e_{4} \\
e_{2} e_{2}=e_{3}\end{array}$ & $\begin{array}{l}e_{1} e_{3}=\alpha e_{4} \\
e_{3} e_{3}=e_{4}\end{array}$ & \\
\hline $\begin{array}{l}\mathfrak{C D}_{92}^{4}(\lambda, \alpha) \\
\lambda \neq 0, \frac{1}{4}\end{array}$ & $:$ & $\begin{array}{l}e_{1} e_{1}=\lambda e_{3}+(1-2 \Theta) e_{4} \\
e_{2} e_{1}=e_{3}-\Theta^{2} \lambda^{-1} e_{4}\end{array}$ & $\begin{array}{l}e_{1} e_{2}=e_{4} \\
e_{2} e_{2}=e_{3}\end{array}$ & $\begin{array}{l}e_{1} e_{3}=\alpha e_{4} \\
e_{3} e_{3}=e_{4}\end{array}$ & \\
\hline
\end{tabular}




\begin{tabular}{|c|c|c|c|c|c|}
\hline $\mathfrak{C} \mathfrak{D}_{93}^{4}(\alpha)$ & $:$ & $\begin{array}{l}e_{1} e_{1}=e_{4} \\
e_{2} e_{2}=e_{3}\end{array}$ & $\begin{array}{l}e_{1} e_{2}=e_{4} \\
e_{2} e_{3}=\alpha e_{4}\end{array}$ & $\begin{array}{l}e_{1} e_{3}=\alpha e_{4} \\
e_{3} e_{3}=e_{4}\end{array}$ & $e_{2} e_{1}=e_{3}+e_{4}$ \\
\hline $\begin{array}{l}\mathfrak{C D}_{94}^{4}(\alpha, \beta) \\
\alpha \neq 0\end{array}$ & $:$ & $\begin{array}{l}e_{1} e_{1}=e_{4} \\
e_{2} e_{1}=e_{3}+\beta e_{4}\end{array}$ & $\begin{array}{l}e_{1} e_{2}=e_{4} \\
e_{2} e_{2}=e_{3}\end{array}$ & $\begin{array}{l}e_{1} e_{3}=\alpha e_{4} \\
e_{3} e_{3}=e_{4}\end{array}$ & \\
\hline $\mathfrak{C} \mathfrak{D}_{95}^{4}(\alpha)$ & $:$ & $\begin{array}{l}e_{1} e_{1}=e_{4} \\
e_{2} e_{2}=e_{3}\end{array}$ & $\begin{array}{l}e_{1} e_{2}=e_{4} \\
e_{2} e_{3}=\alpha e_{4}\end{array}$ & $\begin{array}{l}e_{1} e_{3}=\alpha e_{4} \\
e_{3} e_{3}=e_{4}\end{array}$ & $e_{2} e_{1}=e_{3}$ \\
\hline $\mathfrak{C} \mathfrak{D}_{96}^{4}(\alpha)$ & : & $\begin{array}{l}e_{1} e_{1}=e_{4} \\
e_{2} e_{2}=e_{3}\end{array}$ & $\begin{array}{l}e_{1} e_{2}=e_{4} \\
e_{2} e_{3}=e_{4}\end{array}$ & $\begin{array}{l}e_{2} e_{1}=e_{3}+\alpha e_{4} \\
e_{3} e_{3}=e_{4}\end{array}$ & \\
\hline $\mathfrak{C} \mathfrak{D}_{97}^{4}(\lambda)$ & $:$ & $\begin{array}{l}e_{1} e_{1}=\lambda e_{3} \\
e_{2} e_{2}=e_{3}\end{array}$ & $\begin{array}{l}e_{1} e_{2}=e_{4} \\
e_{2} e_{3}=e_{4}\end{array}$ & $\begin{aligned} e_{1} e_{3} & =\Theta e_{4} \\
e_{3} e_{3} & =e_{4}\end{aligned}$ & $e_{2} e_{1}=e_{3}-e_{4}$ \\
\hline $\begin{array}{l}\mathfrak{C} \mathfrak{D}_{98}^{4}(\lambda) \\
\lambda \neq \frac{1}{4}\end{array}$ & $\overline{:}$ & $\begin{array}{l}e_{1} e_{1}=\lambda e_{3} \\
e_{2} e_{2}=e_{3}\end{array}$ & $\begin{array}{l}e_{1} e_{2}=e_{4} \\
e_{2} e_{3}=e_{4}\end{array}$ & $\begin{array}{l}e_{1} e_{3}=(1-\Theta) e_{4} \\
e_{3} e_{3}=e_{4}\end{array}$ & $e_{2} e_{1}=e_{3}-e_{4}$ \\
\hline $\begin{array}{l}\mathfrak{C D D}_{99}^{4}(\alpha) \\
\alpha \neq 1\end{array}$ & $:$ & $\begin{array}{l}e_{1} e_{2}=e_{4} \\
e_{2} e_{2}=e_{3}\end{array}$ & $\begin{array}{l}e_{1} e_{3}=e_{4} \\
e_{2} e_{3}=\alpha e_{4}\end{array}$ & $\begin{array}{l}e_{2} e_{1}=e_{3}-e_{4} \\
e_{3} e_{3}=e_{4}\end{array}$ & \\
\hline $\begin{array}{l}\mathfrak{C D}_{100}^{4}(\alpha) \\
\alpha \notin\left\{0, \frac{1}{2}\right\}\end{array}$ & : & $\begin{array}{l}e_{1} e_{1}=\frac{1}{4} e_{3} \\
e_{2} e_{2}=e_{3}\end{array}$ & $\begin{array}{l}e_{1} e_{2}=e_{4} \\
e_{2} e_{3}=2 \alpha e_{4}\end{array}$ & $\begin{array}{l}e_{1} e_{3}=\alpha e_{4} \\
e_{3} e_{3}=e_{4}\end{array}$ & $e_{2} e_{1}=e_{3}-e_{4}$ \\
\hline $\mathfrak{C} \mathfrak{D}_{101}^{4}(\alpha, \beta)$ & $:$ & $\begin{array}{l}e_{1} e_{2}=e_{4} \\
e_{2} e_{2}=e_{3}\end{array}$ & $\begin{array}{l}e_{1} e_{3}=\alpha e_{4} \\
e_{2} e_{3}=\beta e_{4}\end{array}$ & $\begin{array}{l}e_{2} e_{1}=e_{3} \\
e_{3} e_{3}=e_{4}\end{array}$ & \\
\hline $\begin{array}{l}\mathfrak{C D}_{102}^{4}(\lambda, \alpha) \\
\lambda \neq 0\end{array}$ & : & $\begin{array}{l}e_{1} e_{1}=\lambda e_{3} \\
e_{2} e_{2}=e_{3}\end{array}$ & $\begin{array}{l}e_{1} e_{2}=e_{4} \\
e_{2} e_{3}=\alpha e_{4}\end{array}$ & $\begin{array}{l}e_{2} e_{1}=e_{3}-e_{4} \\
e_{3} e_{3}=e_{4}\end{array}$ & \\
\hline $\mathfrak{C} \mathfrak{D}_{103}^{4}$ & $:$ & $e_{1} e_{2}=e_{4}$ & $e_{2} e_{1}=e_{3}-e_{4}$ & $e_{2} e_{2}=e_{3}$ & $e_{3} e_{3}=e_{4}$ \\
\hline $\mathfrak{C} \mathfrak{D}_{104}^{4}$ & : & $\begin{array}{l}e_{1} e_{3}=e_{4} \\
e_{2} e_{3}=e_{4}\end{array}$ & $\begin{array}{l}e_{2} e_{1}=e_{3}+e_{4} \\
e_{3} e_{3}=e_{4}\end{array}$ & $e_{2} e_{2}=e_{3}$ & \\
\hline $\begin{array}{l}\mathfrak{C D}_{105}^{4}(\lambda, \alpha, \beta) \\
\lambda \neq 0, \alpha \neq 0\end{array}$ & : & $\begin{array}{l}e_{1} e_{1}=\lambda e_{3} \\
e_{2} e_{2}=e_{3}\end{array}$ & $\begin{aligned} e_{1} e_{3} & =e_{4} \\
e_{2} e_{3} & =\beta e_{4}\end{aligned}$ & $\begin{array}{l}e_{2} e_{1}=e_{3}+\alpha e_{4} \\
e_{3} e_{3}=e_{4}\end{array}$ & \\
\hline $\mathfrak{C} \mathfrak{D}_{106}^{4}(\alpha)$ & 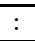 & $e_{1} e_{3}=e_{4}$ & $e_{2} e_{1}=e_{3}+\alpha e_{4}$ & $e_{2} e_{2}=e_{3}$ & $e_{3} e_{3}=e_{4}$ \\
\hline $\mathfrak{C D}_{107}^{4}(\lambda)$ & $\overline{:}$ & $\begin{array}{l}e_{1} e_{1}=\lambda e_{3} \\
e_{2} e_{2}=e_{3}\end{array}$ & $\begin{array}{l}e_{1} e_{3}=\Theta e_{4} \\
e_{2} e_{3}=e_{4}\end{array}$ & $\begin{array}{l}e_{2} e_{1}=e_{3} \\
e_{3} e_{3}=e_{4}\end{array}$ & \\
\hline $\begin{array}{l}\mathfrak{C D}_{108}^{4}(\lambda) \\
\lambda \notin\left\{0, \frac{1}{4}\right\}\end{array}$ & $\overline{:}$ & $\begin{array}{l}e_{1} e_{1}=\lambda e_{3} \\
e_{2} e_{2}=e_{3}\end{array}$ & $\begin{array}{l}e_{1} e_{3}=(1-\Theta) e_{4} \\
e_{2} e_{3}=e_{4}\end{array}$ & $\begin{array}{l}e_{2} e_{1}=e_{3} \\
e_{3} e_{3}=e_{4}\end{array}$ & \\
\hline $\mathfrak{C D}_{109}^{4}(\lambda, \alpha)$ & 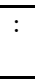 & $\begin{array}{l}e_{1} e_{1}=\lambda e_{3} \\
e_{2} e_{2}=e_{3}\end{array}$ & $\begin{array}{l}e_{2} e_{1}=e_{3}+e_{4} \\
e_{2} e_{3}=\alpha e_{4}\end{array}$ & $e_{3} e_{3}=e_{4}$ & \\
\hline $\mathfrak{C} \mathfrak{D}_{110}^{4}(\lambda)$ & $\overline{:}$ & $\begin{array}{l}e_{1} e_{1}=\lambda e_{3} \\
e_{2} e_{3}=e_{4}\end{array}$ & $\begin{array}{l}e_{2} e_{1}=e_{3} \\
e_{3} e_{3}=e_{4}\end{array}$ & $e_{2} e_{2}=e_{3}$ & \\
\hline $\mathfrak{C} \mathfrak{D}_{111}^{4}(\lambda)$ & $:$ & $e_{1} e_{1}=\lambda e_{3}$ & $e_{2} e_{1}=e_{3}$ & $e_{2} e_{2}=e_{3}$ & $e_{3} e_{3}=e_{4}$ \\
\hline $\mathfrak{C D}_{112}^{4}(\lambda, \alpha, \beta, \gamma)$ & $:$ & $\begin{array}{l}e_{1} e_{1}=\lambda e_{3}+e_{4} \\
e_{2} e_{2}=e_{3}\end{array}$ & $\begin{array}{l}e_{1} e_{3}=\alpha e_{4} \\
e_{2} e_{3}=\gamma e_{4}\end{array}$ & $\begin{array}{l}e_{2} e_{1}=e_{3}+\beta e_{4} \\
e_{3} e_{3}=e_{4}\end{array}$ & \\
\hline $\mathbf{N}_{01}^{4}(\alpha)$ & 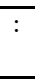 & $\begin{array}{l}e_{1} e_{1}=e_{2} \\
e_{2} e_{1}=e_{4}\end{array}$ & $\begin{array}{l}e_{1} e_{2}=\alpha e_{4} \\
e_{2} e_{2}=e_{3}\end{array}$ & $e_{1} e_{3}=e_{4}$ & \\
\hline$\overline{\mathbf{N}_{02}^{4}(\alpha, \beta, \gamma, \delta)}$ & $:$ & $\begin{array}{l}e_{1} e_{1}=e_{2} \\
e_{2} e_{2}=e_{3}\end{array}$ & $\begin{array}{l}e_{1} e_{2}=\alpha e_{4} \\
e_{2} e_{3}=\delta e_{4}\end{array}$ & $\begin{array}{l}e_{1} e_{3}=\gamma e_{4} \\
e_{3} e_{2}=e_{4}\end{array}$ & $\begin{array}{l}e_{2} e_{1}=\beta e_{4} \\
e_{3} e_{3}=e_{4}\end{array}$ \\
\hline $\mathbf{N}_{03}^{4}(\alpha)$ & $:$ & $\begin{array}{l}e_{1} e_{1}=e_{2} \\
e_{2} e_{2}=e_{3}\end{array}$ & $\begin{array}{l}e_{1} e_{2}=\alpha e_{4} \\
e_{2} e_{3}=e_{4}\end{array}$ & $e_{1} e_{3}=e_{4}$ & \\
\hline $\mathbf{N}_{04}^{4}(\alpha, \beta, \gamma)$ & : & $\begin{array}{l}e_{1} e_{1}=e_{2} \\
e_{2} e_{2}=e_{3}\end{array}$ & $\begin{array}{l}e_{1} e_{2}=\alpha e_{4} \\
e_{2} e_{3}=e_{4}\end{array}$ & $\begin{array}{l}e_{1} e_{3}=\gamma e_{4} \\
e_{3} e_{3}=e_{4}\end{array}$ & $e_{2} e_{1}=\beta e_{4}$ \\
\hline $\mathbf{N}_{05}^{4}(\alpha, \beta)$ & $:$ & $\begin{array}{l}e_{1} e_{1}=e_{2} \\
e_{2} e_{1}=\beta e_{4}\end{array}$ & $\begin{array}{l}e_{1} e_{2}=\alpha e_{4} \\
e_{2} e_{2}=e_{3}\end{array}$ & $\begin{array}{l}e_{1} e_{3}=e_{4} \\
e_{3} e_{3}=e_{4}\end{array}$ & \\
\hline $\mathbf{N}_{06}^{4}(\alpha)$ & : & $\begin{array}{l}e_{1} e_{1}=e_{2} \\
e_{2} e_{2}=e_{3}\end{array}$ & $\begin{array}{l}e_{1} e_{2}=\alpha e_{4} \\
e_{3} e_{3}=e_{4}\end{array}$ & $e_{2} e_{1}=e_{4}$ & \\
\hline$\overline{\mathbf{N}_{07}^{4}}$ & $:$ & $e_{1} e_{1}=e_{2}$ & $e_{1} e_{2}=e_{4}$ & $e_{1} e_{3}=e_{4}$ & $e_{2} e_{2}=e_{3}$ \\
\hline$\overline{\mathbf{N}_{08}^{4}(\alpha, \beta)}$ & : & $\begin{array}{l}e_{1} e_{1}=e_{2} \\
e_{2} e_{1}=\alpha e_{4}\end{array}$ & $\begin{array}{l}e_{1} e_{2}=e_{4} \\
e_{2} e_{2}=e_{3}\end{array}$ & $\begin{array}{l}e_{1} e_{3}=\beta e_{4} \\
e_{3} e_{1}=e_{4}\end{array}$ & \\
\hline $\mathbf{N}_{09}^{4}(\alpha, \beta)$ & $:$ & $\begin{array}{l}e_{1} e_{1}=e_{2} \\
e_{2} e_{2}=e_{3}\end{array}$ & $\begin{array}{l}e_{1} e_{2}=\alpha e_{4} \\
e_{2} e_{3}=e_{4}\end{array}$ & $\begin{array}{l}e_{1} e_{3}=\beta e_{4} \\
e_{3} e_{1}=e_{4}\end{array}$ & \\
\hline
\end{tabular}




\begin{tabular}{|c|c|c|c|c|c|}
\hline $\mathbf{N}_{10}^{4}$ & $:$ & $e_{1} e_{1}=e_{2}$ & $e_{1} e_{2}=e_{4}$ & $e_{2} e_{2}=e_{3}$ & $e_{2} e_{3}=e_{4}$ \\
\hline $\mathbf{N}_{11}^{4}$ & $:$ & $e_{1} e_{1}=e_{2}$ & $e_{1} e_{2}=e_{4}$ & $e_{2} e_{2}=e_{3}$ & $e_{3} e_{3}=e_{4}$ \\
\hline \multirow[t]{2}{*}{$\mathbf{N}_{12}^{4}(\alpha)$} & : & $e_{1} e_{1}=e_{2}$ & $e_{1} e_{3}=\alpha e_{4}$ & $e_{2} e_{1}=e_{4}$ & \\
\hline & & $e_{2} e_{2}=e_{3}$ & $e_{3} e_{1}=e_{4}$ & & \\
\hline \multirow[t]{2}{*}{$\mathbf{N}_{13}^{4}(\alpha, \beta, \gamma)$} & : & $e_{1} e_{1}=e_{2}$ & $e_{1} e_{3}=\beta e_{4}$ & $e_{2} e_{1}=\alpha e_{4}$ & $e_{2} e_{2}=e_{3}$ \\
\hline & & $e_{2} e_{3}=\gamma e_{4}$ & $e_{3} e_{1}=e_{4}$ & $e_{3} e_{2}=e_{4}$ & \\
\hline \multirow[t]{2}{*}{$\mathbf{N}_{14}^{4}(\alpha, \beta)$} & : & $e_{1} e_{1}=e_{2}$ & $e_{1} e_{3}=e_{4}$ & $e_{2} e_{1}=\alpha e_{4}$ & \\
\hline & & $e_{2} e_{2}=e_{3}$ & $e_{2} e_{3}=\beta e_{4}$ & $e_{3} e_{2}=e_{4}$ & \\
\hline \multirow[t]{2}{*}{$\mathbf{N}_{15}^{4}(\alpha)$} & : & $e_{1} e_{1}=e_{2}$ & $e_{2} e_{1}=e_{4}$ & $e_{2} e_{2}=e_{3}$ & \\
\hline & & $e_{2} e_{3}=\alpha e_{4}$ & $e_{3} e_{2}=e_{4}$ & & \\
\hline $\mathbf{N}_{16}^{4}(\alpha)$ & $:$ & $e_{1} e_{1}=e_{2}$ & $e_{1} e_{3}=\alpha e_{4}$ & $e_{2} e_{2}=e_{3}$ & $e_{3} e_{1}=e_{4}$ \\
\hline $\mathbf{N}_{17}^{4}$ & $:$ & $e_{1} e_{1}=e_{2}$ & $e_{1} e_{3}=e_{4}$ & $e_{2} e_{2}=e_{3}$ & \\
\hline $\mathbf{N}_{18}^{4}$ & $:$ & $e_{1} e_{1}=e_{2}$ & $e_{2} e_{2}=e_{3}$ & $e_{2} e_{3}=e_{4}$ & \\
\hline $\mathbf{N}_{19}^{4}(\alpha)$ & $:$ & $e_{1} e_{1}=e_{2}$ & $e_{2} e_{2}=e_{3}$ & $e_{2} e_{3}=\alpha e_{4}$ & $e_{3} e_{2}=e_{4}$ \\
\hline $\mathbf{N}_{20}^{4}$ & $:$ & $e_{1} e_{1}=e_{2}$ & $e_{2} e_{2}=e_{3}$ & $e_{3} e_{3}=e_{4}$ & \\
\hline \multirow[t]{2}{*}{$\mathbf{N}_{21}^{4}(\alpha, \beta)$} & : & $e_{1} e_{1}=e_{2}$ & $e_{1} e_{2}=\alpha e_{4}$ & $e_{1} e_{3}=e_{4}$ & \\
\hline & & $e_{2} e_{1}=e_{3}+\beta e_{4}$ & $e_{2} e_{2}=e_{3}$ & & \\
\hline \multirow[t]{2}{*}{$\mathbf{N}_{22}^{4}(\alpha, \beta, \gamma)$} & : & $e_{1} e_{1}=e_{2}$ & $e_{1} e_{2}=\alpha e_{4}$ & $e_{1} e_{3}=\gamma e_{4}$ & \\
\hline & & $e_{2} e_{1}=e_{3}+\beta e_{4}$ & $e_{2} e_{2}=e_{3}$ & $e_{3} e_{1}=e_{4}$ & \\
\hline \multirow[t]{2}{*}{$\mathbf{N}_{23}^{4}(\alpha, \beta, \gamma)$} & $:$ & $e_{1} e_{1}=e_{2}$ & $e_{1} e_{2}=\alpha e_{4}$ & $e_{1} e_{3}=\beta e_{4}$ & $e_{2} e_{1}=e_{3}$ \\
\hline & & $e_{2} e_{2}=e_{3}$ & $e_{2} e_{3}=e_{4}$ & $e_{3} e_{1}=\gamma e_{4}$ & \\
\hline \multirow[t]{2}{*}{$\mathbf{N}_{24}^{4}(\alpha, \beta, \gamma, \delta)$} & : & $e_{1} e_{1}=e_{2}$ & $e_{1} e_{3}=\beta e_{4}$ & $e_{2} e_{1}=e_{3}+\alpha e_{4}$ & $e_{2} e_{2}=e_{3}$ \\
\hline & & $e_{2} e_{3}=\delta e_{4}$ & $e_{3} e_{1}=\gamma e_{4}$ & $e_{3} e_{2}=e_{4}$ & \\
\hline \multirow[t]{2}{*}{$\mathbf{N}_{25}^{4}(\alpha, \beta, \gamma, \delta, \varepsilon)$} & $:$ & $e_{1} e_{1}=e_{2}$ & $e_{1} e_{2}=\alpha e_{4}$ & $e_{2} e_{1}=e_{3}+\beta e_{4}$ & $e_{2} e_{2}=e_{3}$ \\
\hline & & $e_{2} e_{3}=\delta e_{4}$ & $e_{3} e_{1}=\gamma e_{4}$ & $e_{3} e_{2}=\varepsilon e_{4}$ & $e_{3} e_{3}=e_{4}$ \\
\hline \multirow{2}{*}{$\mathbf{N}_{26}^{4}(\alpha)$} & $:$ & $e_{1} e_{1}=e_{2}$ & $e_{1} e_{2}=e_{4}$ & $e_{1} e_{3}=-\alpha e_{4}$ & \\
\hline & & $e_{2} e_{1}=e_{3}$ & $e_{2} e_{2}=\alpha e_{4}$ & $e_{3} e_{1}=(1+2 \alpha) e_{4}$ & \\
\hline \multirow[t]{2}{*}{$\mathbf{N}_{27}^{4}(\alpha, \beta)$} & $:$ & $e_{1} e_{1}=e_{2}$ & $e_{1} e_{2}=\alpha e_{4}$ & $e_{2} e_{1}=e_{3}$ & \\
\hline & & $e_{2} e_{2}=\beta e_{4}$ & $e_{2} e_{3}=e_{4}$ & $e_{3} e_{1}=e_{4}$ & \\
\hline \multirow[t]{2}{*}{$\mathbf{N}_{28}^{4}(\alpha, \beta, \gamma)$} & : & $e_{1} e_{1}=e_{2}$ & $e_{1} e_{2}=\alpha e_{4}$ & $e_{2} e_{1}=e_{3}$ & $e_{2} e_{2}=\beta e_{4}$ \\
\hline & & $e_{2} e_{3}=e_{4}$ & $e_{3} e_{1}=\gamma e_{4}$ & $e_{3} e_{3}=e_{4}$ & \\
\hline \multirow[t]{2}{*}{$\mathbf{N}_{29}^{4}(\alpha, \beta)$} & $:$ & $e_{1} e_{1}=e_{2}$ & $e_{1} e_{2}=\alpha e_{4}$ & $e_{2} e_{2}=\beta e_{4}$ & \\
\hline & & $e_{2} e_{1}=e_{3}$ & $e_{3} e_{1}=e_{4}$ & $e_{3} e_{3}=e_{4}$ & \\
\hline \multirow[t]{2}{*}{$\mathbf{N}_{30}^{4}(\alpha)$} & : & $e_{1} e_{1}=e_{2}$ & $e_{1} e_{2}=\alpha e_{4}$ & $e_{2} e_{1}=e_{3}$ & \\
\hline & & $e_{2} e_{2}=e_{4}$ & $e_{2} e_{3}=e_{4}$ & & \\
\hline \multirow[t]{2}{*}{$\mathbf{N}_{31}^{4}(\alpha)$} & : & $e_{1} e_{1}=e_{2}$ & $e_{1} e_{2}=\alpha e_{4}$ & $e_{2} e_{1}=e_{3}$ & \\
\hline & & $e_{2} e_{2}=e_{4}$ & $e_{3} e_{3}=e_{4}$ & & \\
\hline $\mathbf{N}_{32}^{4}$ & $:$ & $e_{1} e_{1}=e_{2}$ & $e_{1} e_{2}=e_{4}$ & $e_{2} e_{1}=e_{3}$ & $e_{2} e_{3}=e_{4}$ \\
\hline $\mathbf{N}_{33}^{4}$ & $:$ & $e_{1} e_{1}=e_{2}$ & $e_{1} e_{2}=e_{4}$ & $e_{2} e_{1}=e_{3}$ & $e_{3} e_{3}=e_{4}$ \\
\hline \multirow[t]{2}{*}{$\mathbf{N}_{34}^{4}(\alpha, \beta)$} & : & $e_{1} e_{1}=e_{2}$ & $e_{1} e_{3}=\beta e_{4}$ & $e_{2} e_{1}=e_{3}$ & \\
\hline & & $e_{2} e_{2}=\alpha e_{4}$ & $e_{3} e_{1}=(1-2 \beta) e_{4}$ & & \\
\hline \multirow[t]{2}{*}{$\mathbf{N}_{35}^{4}(\alpha)$} & : & $e_{1} e_{1}=e_{2}$ & $e_{1} e_{3}=\alpha e_{4}$ & $e_{2} e_{1}=e_{3}$ & \\
\hline & & $e_{2} e_{2}=e_{4}$ & $e_{3} e_{1}=-2 \alpha e_{4}$ & $e_{3} e_{2}=e_{4}$ & \\
\hline \multirow{2}{*}{$\begin{array}{l}\mathbf{N}_{36}^{4}(\alpha, \beta) \\
\beta \neq 0\end{array}$} & : & $e_{1} e_{1}=e_{2}$ & $e_{2} e_{1}=e_{3}$ & $e_{2} e_{2}=\alpha e_{4}$ & \\
\hline & & $e_{2} e_{3}=\beta e_{4}$ & $e_{3} e_{1}=e_{4}$ & $e_{3} e_{2}=e_{4}$ & \\
\hline \multirow{2}{*}{$\begin{array}{l}\mathbf{N}_{37}^{4}(\alpha) \\
\alpha \neq 0\end{array}$} & : & $e_{1} e_{1}=e_{2}$ & $e_{2} e_{1}=e_{3}$ & $e_{2} e_{2}=e_{4}$ & \\
\hline & & $e_{2} e_{3}=\alpha e_{4}$ & $e_{3} e_{2}=e_{4}$ & & \\
\hline $\mathbf{N}_{38}^{4}$ & : & $e_{1} e_{1}=e_{2}$ & $e_{1} e_{3}=e_{4}$ & $e_{2} e_{1}=e_{3}$ & \\
\hline & & $e_{3} e_{1}=-2 e_{4}$ & $e_{3} e_{2}=e_{4}$ & & \\
\hline $\mathbf{N}_{39}^{4}$ & $:$ & $e_{1} e_{1}=e_{2}$ & $e_{2} e_{1}=e_{3}$ & $e_{2} e_{3}=e_{4}$ & \\
\hline $\mathbf{N}_{40}^{4}(\alpha)$ & $:$ & $e_{1} e_{1}=e_{2}$ & $e_{2} e_{1}=e_{3}$ & $e_{2} e_{3}=\alpha e_{4}$ & $e_{3} e_{2}=e_{4}$ \\
\hline $\mathbf{N}_{41}^{4}$ & $:$ & $e_{1} e_{1}=e_{2}$ & $e_{2} e_{1}=e_{3}$ & $e_{3} e_{3}=e_{4}$ & \\
\hline $\mathbf{N}_{42}^{4}(\lambda, \alpha)$ & : & $e_{1} e_{1}=e_{2}$ & $e_{1} e_{2}=e_{3}$ & $e_{1} e_{3}=(\alpha(\lambda-2)+$ & $e_{4}$ \\
\hline$\lambda \neq 1$ & & $e_{2} e_{1}=\lambda e_{3}+e_{4}$ & $e_{2} e_{2}=\frac{(\lambda+1)\left(\left(\lambda^{2}-1\right.\right.}{1-\lambda}$ & $\frac{\lambda+2)}{2} e_{4}$ & $e_{3} e_{1}=(\alpha(1-2 \lambda)-2) e_{4}$ \\
\hline
\end{tabular}




\begin{tabular}{|c|c|c|c|c|c|}
\hline $\mathbf{N}_{43}^{4}(\alpha, \beta)$ & $:$ & $\begin{array}{l}e_{1} e_{1}=e_{2} \\
e_{2} e_{2}=e_{4}\end{array}$ & $\begin{array}{l}e_{1} e_{2}=e_{3} \\
e_{2} e_{3}=-\frac{1}{2} e_{4}\end{array}$ & $\begin{array}{l}e_{1} e_{3}=-\frac{5 \alpha}{2} e_{4} \\
e_{3} e_{1}=2 \alpha e_{4}\end{array}$ & $\begin{array}{l}e_{2} e_{1}=-\frac{1}{2} e_{3}+\beta e_{4} \\
e_{3} e_{2}=e_{4}\end{array}$ \\
\hline $\mathbf{N}_{44}^{4}(\alpha)$ & : & $\begin{array}{l}e_{1} e_{1}=e_{2} \\
e_{2} e_{3}=-\frac{1}{2} e_{4}\end{array}$ & $\begin{array}{l}e_{1} e_{2}=e_{3} \\
e_{3} e_{1}=2 \alpha e_{4}\end{array}$ & $\begin{array}{l}e_{1} e_{3}=-\frac{5 \alpha}{2} e_{4} \\
e_{3} e_{2}=e_{4}\end{array}$ & $e_{2} e_{1}=-\frac{1}{2} e_{3}+e_{4}$ \\
\hline $\mathbf{N}_{45}^{4}(\lambda, \alpha, \beta)$ & $:$ & $\begin{array}{l}e_{1} e_{1}=e_{2} \\
e_{2} e_{1}=\lambda e_{3}\end{array}$ & $\begin{array}{l}e_{1} e_{2}=e_{3} \\
e_{2} e_{2}=\beta e_{4}\end{array}$ & $\begin{array}{l}e_{1} e_{3}=(\alpha(\lambda-2)+1) \\
e_{3} e_{1}=(\alpha(1-2 \lambda)-\end{array}$ & $\begin{array}{l}e_{4} \\
2) e_{4}\end{array}$ \\
\hline $\begin{array}{l}\mathbf{N}_{46}^{4}(\lambda, \alpha) \\
\lambda \neq-\frac{1}{2}\end{array}$ & : & $\begin{array}{l}e_{1} e_{1}=e_{2} \\
e_{2} e_{2}=e_{4}\end{array}$ & $\begin{array}{l}e_{1} e_{2}=e_{3} \\
e_{2} e_{3}=-\frac{1}{2} e_{4}\end{array}$ & $\begin{array}{l}e_{1} e_{3}=\alpha(\lambda-2) e_{4} \\
e_{3} e_{1}=\alpha(1-2 \lambda) e_{4}\end{array}$ & $\begin{array}{l}e_{2} e_{1}=\lambda e_{3} \\
e_{3} e_{2}=e_{4}\end{array}$ \\
\hline $\mathbf{N}_{47}^{4}$ & $:$ & $\begin{array}{l}e_{1} e_{1}=e_{2} \\
e_{2} e_{3}=-\frac{1}{2} e_{4}\end{array}$ & $\begin{array}{l}e_{1} e_{2}=e_{3} \\
e_{3} e_{1}=2 e_{4}\end{array}$ & $\begin{array}{l}e_{1} e_{3}=-\frac{5}{2} e_{4} \\
e_{3} e_{2}=e_{4}\end{array}$ & $e_{2} e_{1}=-\frac{1}{2} e_{3}$ \\
\hline $\begin{array}{l}\mathbf{N}_{48}^{4}(\lambda, \alpha, \beta) \\
\lambda \neq-\frac{1}{2}\end{array}$ & $:$ & $\begin{array}{l}e_{1} e_{1}=e_{2} \\
e_{2} e_{2}=\beta e_{4}\end{array}$ & $\begin{array}{l}e_{1} e_{2}=e_{3} \\
e_{2} e_{3}=\lambda e_{4}\end{array}$ & $\begin{array}{l}e_{1} e_{3}=e_{4} \\
e_{3} e_{1}=-2 e_{4}\end{array}$ & $\begin{array}{l}e_{2} e_{1}=\lambda e_{3}+\alpha e_{4} \\
e_{3} e_{2}=e_{4}\end{array}$ \\
\hline $\mathbf{N}_{49}^{4}(\alpha, \beta)$ & $:$ & $\begin{array}{l}e_{1} e_{1}=e_{2} \\
e_{2} e_{1}=-e_{3}+\alpha e_{4} \\
e_{3} e_{1}=-2 \beta e_{4}\end{array}$ & $\begin{array}{l}e_{1} e_{2}=e_{3} \\
e_{2} e_{2}=e_{4} \\
e_{3} e_{2}=e_{4}\end{array}$ & $\begin{array}{l}e_{1} e_{3}=\beta e_{4} \\
e_{2} e_{3}=e_{4} \\
e_{3} e_{3}=e_{4}\end{array}$ & \\
\hline $\begin{array}{l}\mathbf{N}_{50}^{4}(\lambda, \alpha, \beta) \\
\lambda \neq-1\end{array}$ & : & $\begin{array}{l}e_{1} e_{1}=e_{2} \\
e_{2} e_{2}=\beta e_{4}\end{array}$ & $\begin{array}{l}e_{1} e_{2}=e_{3} \\
e_{3} e_{1}=-2 e_{4}\end{array}$ & $\begin{array}{l}e_{1} e_{3}=e_{4} \\
e_{3} e_{3}=e_{4}\end{array}$ & $e_{2} e_{1}=\lambda e_{3}+\alpha e_{4}$ \\
\hline $\begin{array}{l}\mathbf{N}_{51}^{4}(\lambda, \alpha) \\
\lambda \neq-\frac{1}{2}\end{array}$ & $:$ & $\begin{array}{l}e_{1} e_{1}=e_{2} \\
e_{2} e_{2}=e_{4}\end{array}$ & $\begin{array}{l}e_{1} e_{2}=e_{3} \\
e_{2} e_{3}=\lambda e_{4}\end{array}$ & $\begin{array}{l}e_{2} e_{1}=\lambda e_{3}+\alpha e_{4} \\
e_{3} e_{2}=e_{4}\end{array}$ & \\
\hline $\begin{array}{l}\mathbf{N}_{52}^{4}(\lambda, \alpha, \beta, \gamma) \\
\gamma \neq 1\end{array}$ & $:$ & $\begin{array}{l}e_{1} e_{1}=e_{2} \\
e_{2} e_{3}=\gamma e_{4}\end{array}$ & $\begin{array}{l}e_{1} e_{2}=e_{3} \\
e_{3} e_{2}=e_{4}\end{array}$ & $\begin{array}{l}e_{2} e_{1}=\lambda e_{3}+\alpha e_{4} \\
e_{3} e_{3}=e_{4}\end{array}$ & $e_{2} e_{2}=\beta e_{4}$ \\
\hline $\mathbf{N}_{53}^{4}(\lambda, \alpha, \beta)$ & $:$ & $\begin{array}{l}e_{1} e_{1}=e_{2} \\
e_{2} e_{2}=\beta e_{4}\end{array}$ & $\begin{array}{l}e_{1} e_{2}=e_{3} \\
e_{2} e_{3}=e_{4}\end{array}$ & $\begin{array}{l}e_{2} e_{1}=\lambda e_{3}+\alpha e_{4} \\
e_{3} e_{3}=e_{4}\end{array}$ & \\
\hline $\begin{array}{l}\mathbf{N}_{54}^{4}(\lambda, \alpha) \\
\lambda \neq-1\end{array}$ & $:$ & $\begin{array}{l}e_{1} e_{1}=e_{2} \\
e_{2} e_{2}=e_{4}\end{array}$ & $\begin{array}{l}e_{1} e_{2}=e_{3} \\
e_{3} e_{3}=e_{4}\end{array}$ & $e_{2} e_{1}=\lambda e_{3}+\alpha e_{4}$ & \\
\hline $\begin{array}{l}\mathbf{N}_{55}^{4}(\lambda) \\
\lambda \neq-\frac{1}{2}\end{array}$ & : & $\begin{array}{l}e_{1} e_{1}=e_{2} \\
e_{2} e_{3}=\lambda e_{4}\end{array}$ & $\begin{array}{l}e_{1} e_{2}=e_{3} \\
e_{3} e_{2}=e_{4}\end{array}$ & $e_{2} e_{1}=\lambda e_{3}+e_{4}$ & \\
\hline $\mathbf{N}_{56}^{4}(\lambda)$ & $:$ & $e_{1} e_{1}=e_{2}$ & $e_{1} e_{2}=e_{3}$ & $e_{2} e_{1}=\lambda e_{3}+e_{4}$ & $e_{3} e_{3}=e_{4}$ \\
\hline $\mathbf{N}_{57}^{4}(\lambda, \alpha)$ & : & $\begin{array}{l}e_{1} e_{1}=e_{2} \\
e_{2} e_{2}=\alpha e_{4}\end{array}$ & $\begin{array}{l}e_{1} e_{2}=e_{3} \\
e_{2} e_{3}=e_{4}\end{array}$ & $\begin{array}{l}e_{1} e_{3}=e_{4} \\
e_{3} e_{1}=-2 e_{4}\end{array}$ & $e_{2} e_{1}=\lambda e_{3}$ \\
\hline $\begin{array}{l}\mathbf{N}_{58}^{4}(\lambda, \alpha, \beta) \\
\beta \notin\left\{-\frac{1}{2}, \lambda\right\} \\
\end{array}$ & $:$ & $\begin{array}{l}e_{1} e_{1}=e_{2} \\
e_{2} e_{2}=\alpha e_{4}\end{array}$ & $\begin{array}{l}e_{1} e_{2}=e_{3} \\
e_{2} e_{3}=\beta e_{4}\end{array}$ & $\begin{array}{l}e_{1} e_{3}=e_{4} \\
e_{3} e_{1}=-2 e_{4}\end{array}$ & $\begin{array}{l}e_{2} e_{1}=\lambda e_{3} \\
e_{3} e_{2}=e_{4}\end{array}$ \\
\hline $\begin{array}{l}\mathbf{N}_{59}^{4}(\alpha, \beta) \\
\alpha \neq 1\end{array}$ & $:$ & $\begin{array}{l}e_{1} e_{1}=e_{2} \\
e_{2} e_{1}=-e_{3} \\
e_{3} e_{1}=-2 \beta e_{4}\end{array}$ & $\begin{array}{l}e_{1} e_{2}=e_{3} \\
e_{2} e_{2}=\alpha e_{4} \\
e_{3} e_{2}=e_{4}\end{array}$ & $\begin{array}{l}e_{1} e_{3}=\beta e_{4} \\
e_{2} e_{3}=e_{4} \\
e_{3} e_{3}=e_{4}\end{array}$ & \\
\hline $\begin{array}{l}\mathbf{N}_{60}^{4}(\alpha) \\
\alpha \neq 0\end{array}$ & $:$ & $\begin{array}{l}e_{1} e_{1}=e_{2} \\
e_{2} e_{2}=\alpha e_{4}\end{array}$ & $\begin{array}{l}e_{1} e_{2}=e_{3} \\
e_{3} e_{1}=-2 e_{4}\end{array}$ & $\begin{array}{l}e_{1} e_{3}=e_{4} \\
e_{3} e_{3}=e_{4}\end{array}$ & $e_{2} e_{1}=-e_{3}$ \\
\hline $\mathbf{N}_{61}^{4}(\lambda)$ & $:$ & $\begin{array}{l}e_{1} e_{1}=e_{2} \\
e_{2} e_{2}=e_{4}\end{array}$ & $\begin{array}{l}e_{1} e_{2}=e_{3} \\
e_{2} e_{3}=e_{4}\end{array}$ & $e_{2} e_{1}=\lambda e_{3}$ & \\
\hline $\begin{array}{l}\mathbf{N}_{62}^{4}(\lambda, \alpha) \\
\alpha \notin\left\{-\frac{1}{2}, \lambda\right\}\end{array}$ & : & $\begin{array}{l}e_{1} e_{1}=e_{2} \\
e_{2} e_{2}=e_{4}\end{array}$ & $\begin{array}{l}e_{1} e_{2}=e_{3} \\
e_{2} e_{3}=\alpha e_{4}\end{array}$ & $\begin{array}{l}e_{2} e_{1}=\lambda e_{3} \\
e_{3} e_{2}=e_{4}\end{array}$ & \\
\hline $\mathbf{N}_{63}^{4}$ & : & $\begin{array}{l}e_{1} e_{1}=e_{2} \\
e_{2} e_{2}=e_{4}\end{array}$ & $\begin{array}{l}e_{1} e_{2}=e_{3} \\
e_{3} e_{3}=e_{4}\end{array}$ & $e_{2} e_{1}=-e_{3}$ & \\
\hline $\mathbf{N}_{64}^{4}(\lambda)$ & $:$ & $e_{1} e_{1}=e_{2}$ & $e_{1} e_{2}=e_{3}$ & $e_{2} e_{1}=\lambda e_{3}$ & $e_{2} e_{3}=e_{4}$ \\
\hline $\mathbf{N}_{65}^{4}(\lambda, \alpha)$ & $:$ & $\begin{array}{l}e_{1} e_{1}=e_{2} \\
e_{2} e_{3}=\alpha e_{4}\end{array}$ & $\begin{array}{l}e_{1} e_{2}=e_{3} \\
e_{3} e_{2}=e_{4}\end{array}$ & $e_{2} e_{1}=\lambda e_{3}$ & \\
\hline $\mathbf{N}_{66}^{4}(\lambda)$ & : & $e_{1} e_{1}=e_{2}$ & $e_{1} e_{2}=e_{3}$ & $e_{2} e_{1}=\lambda e_{3}$ & $e_{3} e_{3}=e_{4}$ \\
\hline
\end{tabular}

\section{All of these algebras are pairwise non-isomorphic, except for the following:}

$\mathbf{D}_{01}^{4}(\lambda, 0, \beta) \cong \mathbf{D}_{02}^{4}(\lambda, 0, \beta) \cong \mathbf{D}_{04}^{4}(\lambda, \beta), \quad \mathbf{D}_{01}^{4}(\lambda, \alpha, 0)_{\alpha \neq-1} \cong \mathbf{D}_{02}^{4}(\lambda, \alpha, 0) \cong \mathbf{D}_{10}^{4}(\lambda, \alpha), \quad \mathbf{D}_{01}^{4}(\lambda,-1,0) \cong \mathbf{D}_{11}^{4}(\lambda, 0)$, $\mathbf{D}_{03}^{4}(\lambda, 0) \cong \mathbf{D}_{09}^{4}(\lambda, 0), \quad \mathbf{D}_{03}^{4}\left(\lambda,(1-\Theta)^{-1}\right)_{\lambda \neq 0} \cong \mathbf{D}_{05}^{4}(\lambda, 0)_{\lambda \neq 0}, \mathbf{D}_{03}^{4}\left(\lambda, \Theta^{-1}\right) \cong \mathbf{D}_{06}^{4}(\lambda, 0), \quad \mathbf{D}_{04}^{4}(\lambda, 0) \cong \mathbf{D}_{10}^{4}(\lambda, 0)$,

$\mathbf{D}_{05}^{4}(1 / 4, \alpha) \cong \mathbf{D}_{06}^{4}(1 / 4, \alpha), \quad \mathbf{D}_{07}^{4}(1 / 4) \cong \mathbf{D}_{08}^{4}(1 / 4), \quad \mathbf{D}_{05}^{4}(0, \alpha) \cong \mathbf{D}_{07}^{4}(0) \cong \mathbf{D}_{23}^{4}(0) \cong \mathbf{D}_{25}^{4}(0) \cong \mathbf{D}_{40}^{4}(0)$,

$\mathbf{D}_{12}^{4}(\lambda, 0) \cong \mathbf{D}_{18}^{4}(\lambda, 0), \quad \mathbf{D}_{12}^{4}(1 / 4, \alpha) \cong \mathbf{D}_{13}^{4}(1 / 4, \alpha), \quad \mathbf{D}_{12}^{4}(0, \alpha)_{\alpha \neq-1} \cong \mathbf{D}_{14}^{4}(0, \alpha), \quad \mathbf{D}_{12}^{4}(0,-1) \cong \mathbf{D}_{17}^{4}(0)$, 


$$
\begin{aligned}
& \mathbf{D}_{13}^{4}(\lambda, 0) \cong \mathbf{D}_{19}^{4}(\lambda, 0), \quad \mathbf{D}_{14}^{4}(\lambda, 0) \cong \mathbf{D}_{20}^{4}(\lambda, 0), \quad \mathbf{D}_{14}^{4}(1 / 4, \alpha) \cong \mathbf{D}_{15}^{4}(1 / 4, \alpha), \quad \mathbf{D}_{15}^{4}(\lambda, 0) \cong \mathbf{D}_{21}^{4}(\lambda, 0), \\
& \mathbf{D}_{18}^{4}(1 / 4, \alpha) \cong \mathbf{D}_{19}^{4}(1 / 4, \alpha), \quad \mathbf{D}_{18}^{4}(0,0) \cong \mathbf{D}_{22}^{4}(0) \cong \mathbf{D}_{24}^{4}(0), \quad \mathbf{D}_{18}^{4}(1 / 4,-1) \cong \mathbf{D}_{19}^{4}(1 / 4,-1) \cong \mathbf{D}_{30}^{4}(1 / 4) \cong \mathbf{D}_{31}^{4}(1 / 4), \\
& \mathbf{D}_{20}^{4}(1 / 4, \alpha) \cong \mathbf{D}_{21}^{4}(1 / 4, \alpha), \quad \mathbf{D}_{20}^{4}(1 / 4,-1) \cong \mathbf{D}_{21}^{4}(1 / 4,-1) \cong \mathbf{D}_{32}^{4}(1 / 4) \cong \mathbf{D}_{33}^{4}(1 / 4) \\
& \mathbf{D}_{22}^{4}(1 / 4) \cong \mathbf{D}_{23}^{4}(1 / 4) \cong \mathbf{D}_{24}^{4}(1 / 4) \cong \mathbf{D}_{25}^{4}(1 / 4) \cong \mathbf{D}_{26}^{4}(1 / 4) \cong \mathbf{D}_{27}^{4}(1 / 4) \cong \mathbf{D}_{28}^{4}(1 / 4) \cong \mathbf{D}_{29}^{4}(1 / 4) \\
& \mathbf{D}_{37}^{4}(1 / 4) \cong \mathbf{D}_{38}^{4}(1 / 4), \quad \mathbf{D}_{39}^{4}(1 / 4) \cong \mathbf{D}_{40}^{4}(1 / 4) \text {. }
\end{aligned}
$$

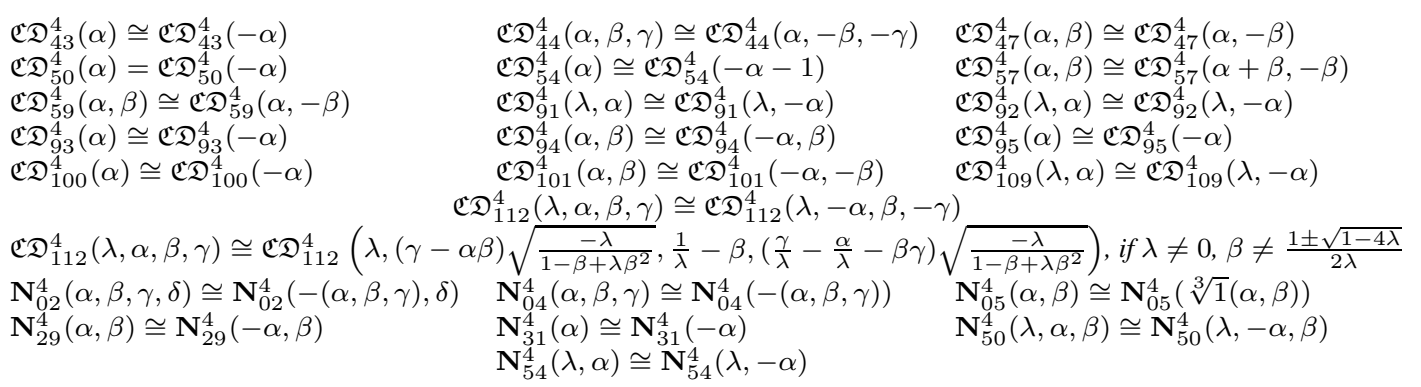

\section{Applications}

3.1. The algebraic classification of 4-dimensional nilpotent Lie-admissible algebras. The variety of Lie-admissible algebras is defined by the following identity

$$
[[x, y], z]+[[y, z], x]+[[z, x], y]=0,
$$

where $[x, y]=x y-y x$. Lie-admissible algebras satisfy the following fundamental property: under the commutator multiplication each Lie-admissible algebra is a Lie algebra.

Corollary 3. Let $\mathbf{A}$ be a complex 4-dimensional nilpotent Lie-admissible algebra. Then $\mathbf{A}$ is isomorphic to an algebra from the list given in Theorem 2.

Proof. Thanks to [15] each 4-dimensional nilpotent anticommutative algebra is Lie. Hence, each 4-dimensional nilpotent algebra is a Lie-admissible algebra.

3.2. The algebraic classification of 4-dimensional nilpotent Alia type algebras. Let $\mathbf{A}$ be an algebra with a certain bilinear multiplication $(x, y) \mapsto x y$, which we call standard. Let us define

$$
\mathrm{T}(x, y, z, \mathrm{P})=\mathrm{P}([x, y], z)+\mathrm{P}([y, z], x)+\mathrm{P}([z, x], y)
$$

where $[x, y]=x y-y x$ and $\mathrm{P}$ is a bilinear multiplication on the same vector space. Now we are ready to introduce Alia type algebras, which appeared in [9, 10]:

(1) the variety of 0-Alia (also known as 0-anti-Lie-admissible) algebras is defined by the identity $\mathrm{T}(x, y, z, \mathbf{P})=0$, where $\mathrm{P}$ is the standard multiplication;

(2) the variety of 1-Alia (also known as 1-anti-Lie-admissible) algebras is defined by the identity $\mathrm{T}(x, y, z, \mathrm{P})=0$, where $\mathrm{P}$ is the Jordan commutator multiplication $\mathrm{P}(x, y)=x y+y x$

(3) the variety of two-sided Alia (also known as anti-Lie-admissible) algebras is defined by the identities $\mathrm{T}\left(x, y, z, \mathrm{P}_{1}\right)=0$ and $\mathrm{T}\left(x, y, z, \mathrm{P}_{2}\right)=0$, where $\mathrm{P}_{1}$ is the standard multiplication and $\mathrm{P}_{2}$ is the opposite multiplication. 
Corollary 4. Let A be a complex 4-dimensional nilpotent 0-Alia (1-Alia, or two sided Alia) algebra. Then $\mathbf{A}$ is isomorphic to an algebra from the list given in Theorem 2 except

$$
\begin{gathered}
\mathfrak{C} \mathfrak{D}_{79}^{4}-\mathfrak{C} \mathfrak{D}_{85}^{4}, \mathfrak{C} \mathfrak{D}_{87}^{4}-\mathfrak{C} \mathfrak{D}_{112}^{4}, \mathbf{N}_{25}^{4}, \mathbf{N}_{28}^{4}, \mathbf{N}_{29}^{4}, \mathbf{N}_{31}^{4}, \mathbf{N}_{33}^{4}, \mathbf{N}_{41}^{4}, \mathbf{N}_{49}^{4}, \mathbf{N}_{50}^{4}(\lambda \neq 1), \\
\mathbf{N}_{52}^{4}(\lambda \neq 1), \mathbf{N}_{53}^{4}(\lambda \neq 1), \mathbf{N}_{54}^{4}(\lambda \neq 1), \mathbf{N}_{56}^{4}(\lambda \neq 1), \mathbf{N}_{59}^{4}, \mathbf{N}_{60}^{4}, \mathbf{N}_{63}^{4}, \mathbf{N}_{66}^{4}(\lambda \neq 1) .
\end{gathered}
$$

Proof. After verification, we have that all 3-dimensional nilpotent algebras are 0-Alia, 1-Alia, and two-sided Alia. Analyzing the cocycles on 3-dimensional algebras we see that the subspaces of 0Alia cocycles, 1-Alia cocycles, and two-sided Alia cocycles coincide and are listed below.

\begin{tabular}{|l|l|}
\hline Algebra & Cocycles \\
\hline $\mathfrak{C} \mathfrak{D}_{01}^{3}, \mathfrak{C} \mathfrak{D}_{04}^{3}(1), \mathfrak{C} \mathfrak{D}_{01}^{3 *}, \mathfrak{C} \mathfrak{D}_{02}^{3 *}$ & $\left\langle\Delta_{i j}\right\rangle_{1 \leq i, j \leq 3}$ \\
\hline $\mathfrak{C} \mathfrak{D}_{02}^{3}, \mathfrak{C} \mathfrak{D}_{03}^{3}, \mathfrak{C} \mathfrak{D}_{04}^{3}(\lambda \neq 1), \mathfrak{C} \mathfrak{D}_{03}^{3 *}, \mathfrak{C} \mathfrak{D}_{04}^{3 *}$ & $\left\langle\Delta_{i j}\right\rangle_{1 \leq i, j \leq 3 ;(i, j) \neq(3,3)}$ \\
\hline
\end{tabular}

It remains to choose the algebras from Theorem 2 that are determined by Alia cocycles.

\section{REFERENCES}

[1] Abdelwahab H., Calderón A.J., Kaygorodov I., The algebraic and geometric classification of nilpotent binary Lie algebras, International Journal of Algebra and Computation, 29 (2019), 6, 1113-1129.

[2] Calderón Martín A., Fernández Ouaridi A., Kaygorodov I., On the classification of bilinear maps with radical of a fixed codimension, Linear and Multilinear Algebra, 2020, DOI:10.1080/03081087.2020.1849001

[3] Camacho L., Kaygorodov I., Lopatkin V., Salim M., The variety of dual Mock-Lie algebras, Communications in Mathematics, 28 (2020), 2, 161-178.

[4] Cicalò S., De Graaf W., Schneider C., Six-dimensional nilpotent Lie algebras, Linear Algebra and its Applications, 436 (2012), 1, 163-189.

[5] Darijani I., Usefi H., The classification of 5-dimensional p-nilpotent restricted Lie algebras over perfect fields. I, Journal of Algebra, 464 (2016), 97-140.

[6] De Graaf W., Classification of 6-dimensional nilpotent Lie algebras over fields of characteristic not 2, Journal of Algebra, 309 (2007), 2, 640-653.

[7] De Graaf W., Classification of nilpotent associative algebras of small dimension, International Journal of Algebra and Computation, 28 (2018), 1, 133-161.

[8] Demir I., Misra K., Stitzinger E., On classification of four-dimensional nilpotent Leibniz algebras, Communications in Algebra, 45 (2017), 3, 1012-1018.

[9] Dzhumadildaev A., Bakirova A., Simple two-sided anti-Lie-admissible algebras, Journal of Mathematical Sciences (N.Y.), 161 (2009), 1, 31-36

[10] Dzhumadildaev A., Tulenbaev K., Exceptional 0-Alia algebras, Journal of Mathematical Sciences (N.Y.), 161 (2009), 1, 37-40.

[11] Fernández Ouaridi A., Kaygorodov I., Khrypchenko M., Volkov Yu., Degenerations of nilpotent algebras, arXiv: 1905.05361

[12] Hegazi A., Abdelwahab H., Classification of five-dimensional nilpotent Jordan algebras, Linear Algebra and its Applications, 494 (2016), 165-218.

[13] Hegazi A., Abdelwahab H., Calderón Martín A., The classification of $n$-dimensional non-Lie Malcev algebras with $(n-4)$-dimensional annihilator, Linear Algebra and its Applications, 505 (2016), 32-56. 
[14] Kaygorodov I., Khrypchenko M., The algebraic classification of nilpotent $\mathfrak{C} \mathfrak{D}$-algebras, Linear and Multilinear algebra, 2020, DOI: 10.1080/03081087.2020.1856030

[15] Kaygorodov I., Khrypchenko M., Lopes S., The algebraic and geometric classification of nilpotent anticommutative algebras, Journal of Pure and Applied Algebra, 224 (2020), 8, 106337.

[16] Kaygorodov I., Khrypchenko M., Popov Yu., The algebraic and geometric classification of nilpotent terminal algebras, Journal of Pure and Applied Algebra, 225 (2021), 6, 106625.

[17] Kaygorodov I., Lopes S., Páez-Guillán P., Non-associative central extensions of null-filiform associative algebras, Journal of Algebra, 560 (2020), 1190-1210.

[18] Kaygorodov I., Páez-Guillán P., Voronin V., The algebraic and geometric classification of nilpotent bicommutative algebras, Algebras and Representation Theory, 23 (2020), 6, 2331-2347.

[19] Mazzola G., The algebraic and geometric classification of associative algebras of dimension five, Manuscripta Mathematica, 27 (1979), 1, 81-101.

[20] Skjelbred T., Sund T., Sur la classification des algebres de Lie nilpotentes, C. R. Acad. Sci. Paris Ser. A-B, 286 (1978), 5, A241-A242. 